2. To: (Receiving Örganization)

Process Technology 73510

3. From: (Originating Organization) Consequence Analysis $8 M 400$

5. Proj./Prog./Dept./Div.: Waste Inventory

8. Originator Remarks:

The attached SD documents the originator's analys is only. It shall not be used as the final or sole document for effecting changes to an authorization basis or safety basis for a facility or activity.

11. Receiver Remarks:
6. Cog. Engr.:
D.A. Himes
7. Purchase order No.:
$N / A$

4. Related EDT No.:

$N / A$

9. Equip./Component No.:

$N / A$

10. System/Bldg./Facility:

Double She 11 Tanks

12. Major Assm. Dwg. No.: $N / A$

13. Permit/Permit Application No.: $N / A$

14. Required Response Date:

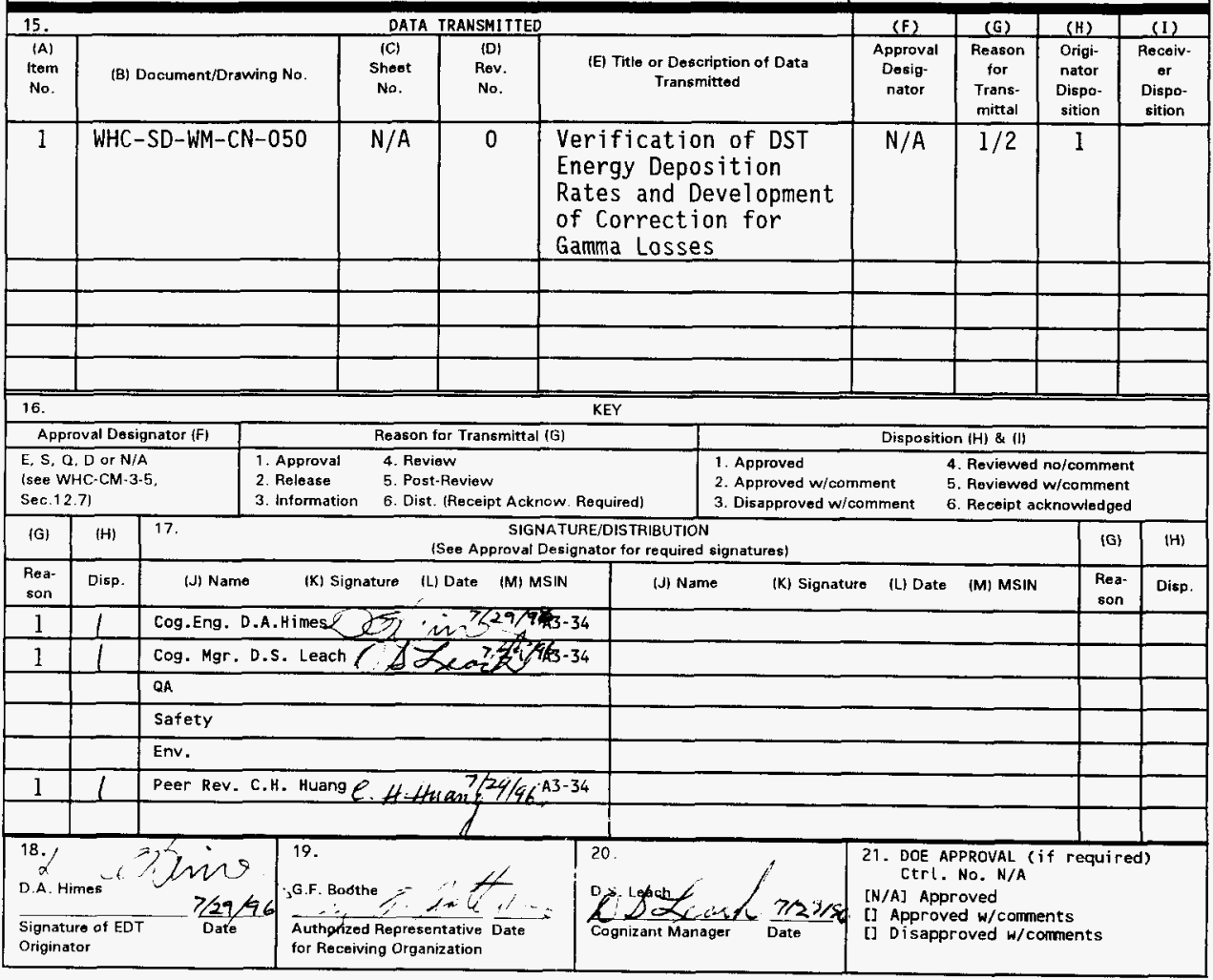




\title{
Verification of DST Energy Deposition Rates and Development of Correction for Gamma Losses
}

\author{
D.A. Himes \\ Westinghouse Hanford Company, Richland, WA 99352 \\ U.S. Department of Energy Contract DE-AC06-87RL10930
EDT/ECN: 156488 UC: 510
Org Code: 8M400 Charge Code: E36251
B\&R Code: H2205 Total Pages: 82

Key Words: Decay Heat, Organic Layer, Double Shell Tanks

Abstract: An independent verification of a series of recently calculated energy deposition rates within dispersed and layered organic waste in double shell tanks was performed. In addition, a simple method was developed to correct for gamma losses from a waste layer when calculating energy deposition rates.

TRADEMARK DISCLAIMER. Reference herein to any specific comercial product, process, or service by trade name, trademark, manufacturer, or otherwise, does not necessarily constitute or imply its endorsement, recommendation, or favoring by the United States Government or any agency thereof or its contractors or subcontractors.

Printed in the United States of America. To obtain copies of this document, contact: WHC/BCS Document Control Services, P.O. Box 1970, Mailstop 46-08, Richland WA 99352, Phone (509) 372-2420. Fax (509) 376-4989.
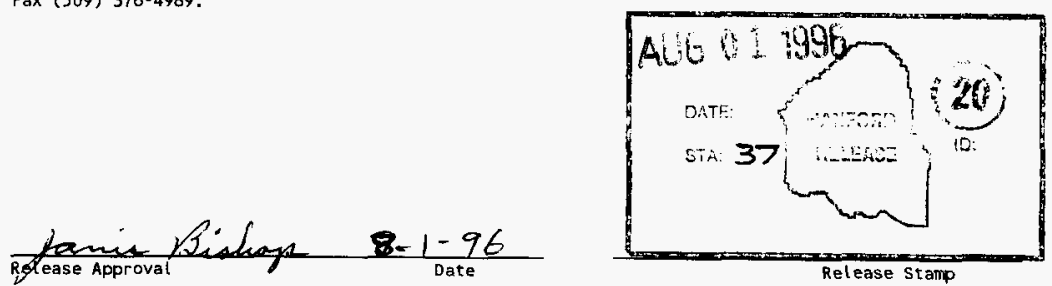

\section{Approved for Public Release}




\title{
VERIFICATION OF DST ENERGY DEPOSITION RATES AND DEVELOPMENT OF CORRECTION FOR GAMMA LOSSES
}

\author{
D.A. Himes
}

$7 / 8 / 96$

Energy deposition rates (dose rates) within dispersed and layered organic waste in a series of double shell tank mixes were recently calculated with the results shown in Attachment 1. An independent verification is required for the tank mixes shown in bold print in the attachment. In addition, it is desired that a simple method be developed for correcting for gamma losses from a waste layer when calculating energy deposition rates.

A cylindrical layer of liquid waste (supernate) is assumed in which an organic component may by uniformly dispersed, or separated as a layer floating on top. No alpha, beta or gamma losses fron the supernate were assumed for the dispersed case. For the layered case the gamma dose rate $5 \mathrm{~cm}$ into the organic layer was calculated using MICROSHIELD?

\section{Dispersed organic cases:}

The initial step in the calculations was to assemble the energy per disintegration carried off by alphas, betas and gammas for each of the isotopes 1 isted in the mix inventories in Attachment 1. These are shown in Table 1 .

Table 1: Energy production per disintegration

\begin{tabular}{cccc} 
Isotope & $\begin{array}{c}\text { Energy per Disintegration } \\
\text { Alpha }\end{array}$ & $\begin{array}{c}\text { MeV/dis) } \\
\text { Geta }\end{array}$ & \\
\hline \hline $\mathrm{C}-14$ & - & $4.95 \mathrm{E}-2$ & - \\
$\mathrm{S} r-90$ & - & $1.96 \mathrm{E}-1$ & - \\
$\mathrm{Y}-90$ & - & $9.35 \mathrm{E}-1$ & - \\
$\mathrm{Tc}-99$ & - & $8.46 \mathrm{E}-2$ & $5.36 \mathrm{E}-7$ \\
$\mathrm{Cs}-137$ & - & $1.71 \mathrm{E}-1$ & - \\
$\mathrm{Ba}-137 \mathrm{~m}$ & - & $6.37 \mathrm{E}-2$ & $5.98 \mathrm{E}-1$ \\
$\mathrm{Eu}-154$ & - & $2.25 \mathrm{E}-1$ & $1.25 \mathrm{E}+0$ \\
$\mathrm{~Np}-237$ & $4.75 \mathrm{E}+0$ & $6.24 \mathrm{E}-2$ & $3.30 \mathrm{E}-2$ \\
$\mathrm{Pu}-238$ & $5.49 \mathrm{E}+0$ & $8.26 \mathrm{E}-3$ & $1.60 \mathrm{E}-3$ \\
$\mathrm{Pu}-239$ & $5.15 \mathrm{E}+0$ & $4.88 \mathrm{E}-3$ & $6.54 \mathrm{E}-4$ \\
$\mathrm{Pu}-240$ & $5.15 \mathrm{E}+0$ & $8.33 \mathrm{E}-3$ & $1.53 \mathrm{E}-3$ \\
$\mathrm{Pu}-241$ & - & $5.23 \mathrm{E}-3$ & - \\
$\mathrm{Am}-241$ & $5.48 \mathrm{E}+0$ & $2.94 \mathrm{E}-2$ & $2.81 \mathrm{E}-2$
\end{tabular}


To obtain the volumetric energy deposition rate it is merely necessary to multiply the energies per disintegration shown in Table 1 by the corresponding isotopic concentrations $(\mathrm{C} i / L)$ and make use of the definition of a $\mathrm{Ci}_{3}$ as $3.7 \mathrm{E}+10 \mathrm{dis} / \mathrm{s}$. This yields $\mathrm{MeV} / \mathrm{s} \cdot \mathrm{L}$. which can be converted to ergs $/ \mathrm{cm}^{3} \cdot \mathrm{h}$ by using the fact that $1 \mathrm{MeV}=1.602 \mathrm{E}-6$ ergs and finally to rads $/ \mathrm{h}$ by dividing by the supernate density and using the definition of a rad as $100 \mathrm{ergs} / \mathrm{g}$ absorbed. The resulting energy deposition rates for the required DST mixes are shown in Table 2. A sample calculation (105AP) is shown in Attachment 2 . The corresponding values which were to be verified are shown in parentheses.

Table 2: Resulting energy deposition (dose) rates for dispersed organics case

\begin{tabular}{|c|c|c|c|c|}
\hline \multirow[b]{2}{*}{ Tank Mix } & \multicolumn{3}{|c|}{ erqs $/ \mathrm{cm}^{3} \cdot h$} & \multirow{2}{*}{$\begin{array}{l}\text { Total Supernate/ } \\
\text { Organic ( } \mathrm{rad} / \mathrm{h})\end{array}$} \\
\hline & Alpha & Beta & Gamma & \\
\hline 105AP & $1.90 \mathrm{E}+1$ & $2.25 E+3$ & $2.13 E+3$ & $3.33 E+1 \quad(3.33 E+1)$ \\
\hline $103 C+105 A P$ & - & $3.09 E+3$ & $7.02 \mathrm{E}+3$ & $9.19 \mathrm{E}+1 \quad(9.17 \mathrm{E}+1)$ \\
\hline 107AP & $4.00 \mathrm{E}-2$ & $1.23 E-3$ & $2.04 \mathrm{E}-3$ & $4.28 \mathrm{E}-4 \quad(4.22 \mathrm{E}-4)$ \\
\hline $103 \mathrm{C}+107 \mathrm{AP}$ & - & $2.61 E+3$ & $6.29 \mathrm{E}+3$ & $8.40 \mathrm{E}+1 \quad(8.40 \mathrm{E}+1)$ \\
\hline $\begin{array}{l}103 \mathrm{U}+105 \mathrm{U}+ \\
109 \mathrm{U}+107 \mathrm{AP}\end{array}$ & - & $1.87 E+4$ & $4.03 E+4$ & $4.37 \mathrm{E}+2 \quad(4.38 \mathrm{E}+2)$ \\
\hline $\begin{array}{l}103 \mathrm{C}+103 \mathrm{U}_{+} \\
105 \mathrm{U}_{+} 109 \mathrm{U}_{+} \\
107 \mathrm{AP}\end{array}$ & - & $1.54 \mathrm{E}+4$ & $3.36 \mathrm{E}+4$ & $3.80 E+2(3.80 E+2)$ \\
\hline 102AY & $2.27 E+1$ & $2.74 \mathrm{E}+3$ & $5.90 E+3$ & $7.59 \mathrm{E}+1 \quad(7.59 \mathrm{E}+1)$ \\
\hline $103 C+102 A Y$ & - & $2.81 E+3$ & $6.16 \mathrm{E}+3$ & $7.94 \mathrm{E}+1 \quad(7.93 \mathrm{E}+1)$ \\
\hline $102 \mathrm{AZ}$ & $2.61 \mathrm{E}+2$ & $2.04 E+4$ & $1.79 \mathrm{E}+4$ & $3.47 E+2 \quad(3.47 E+2)$ \\
\hline
\end{tabular}

There is some variation in the deposition rates attributed to the individual radiative components due to differences in the way the energy was apportioned, possible differences in the data, and (most significantly) in whether minor components were neglected (e.g., B contributions from a primary $\alpha$ emitter). The total dose rates in the supernate/organic dispersion, however, seemed to agree we11 in every case. 


\section{Layered orqanic cases:}

The layered organic cases were calculated using the MICROSHIELD 3.12 code (Grove 1988) with a simple cylindrical geometry. The supernate layer density, thickness and diameter were taken from the information in Attachment 1 . A typical geometry set-up is shown in the sample calculation in Attachment 2 . The MICROSHIELD files for all the mixes are 1isted for reference in Attachment 3 . The resulting dose rates at a point $5 \mathrm{~cm}$ into the organic layer are shown in Table 3. The corresponding values which were to be verified are shown in parentheses.

Table 3: Dose rates $5 \mathrm{~cm}$ into the organic layer

\begin{tabular}{cc} 
Tank Mix & Dose Rate $(\mathrm{rad} / \mathrm{h})$ \\
\hline \hline $105 \mathrm{AP}$ & $6.13 \mathrm{E}+0(6.18 \mathrm{E}+0)$ \\
$103 \mathrm{C}+105 \mathrm{AP}$ & $2.78 \mathrm{E}+1(2.78 \mathrm{E}+1)$ \\
$107 \mathrm{AP}$ & $6.98 \mathrm{E}-6(4.46 \mathrm{E}-6)$ \\
$103 \mathrm{C}+107 \mathrm{AP}$ & $2.59 \mathrm{E}+1(2.58 \mathrm{E}+1)$ \\
$103 \mathrm{U}+105 U_{+}$ & \\
$109 U_{+107 \mathrm{AP}}$ & $1.22 \mathrm{E}+2(1.34 \mathrm{E}+2)$ \\
$103 \mathrm{C}+103 U_{+}$ & \\
$105 \mathrm{U}+109 U_{+}$ & \\
$107 \mathrm{AP}$ & $1.10 \mathrm{E}+2(1.31 \mathrm{E}+2)$ \\
$102 \mathrm{AY}$ & $2.25 \mathrm{E}+1(2.77 \mathrm{E}+1)$ \\
$103 \mathrm{C}+102 \mathrm{AY}$ & $2.55 \mathrm{E}+1(3.26 \mathrm{E}+1)$ \\
$102 \mathrm{AZ}$ & $6.70 \mathrm{E}+1(7.75 \mathrm{E}+1)$
\end{tabular}

In general the results follow pretty well although there are some differences, possible due to differences in the details of the code set-up.

\section{Correction for gamma losses:}

In an infinite medium in which a radioactive material is uniformly distributed (constant concentration) elementary thermodynamic considerations require that the power absorbed at each point must be equal to the power generated at the same point and the power density must be uniform throughout the medium. This 
is the assumption made in the above calculations where the losses from the finite medium were assumed to be zero, i.e., an infinite medium was assumed. For very short range radiation (alphas and betas) this is a good assumption unless the medium is very small. For gamma heating, however, the size of the medium can easily be comparable to the range of the radiation leading to significant energy losses due to leakage from the boundaries of the medium. In this case, the infinite medium assumption could produce a significant overprediction of the average gamma heating in the material. Since the material of interest here is in the form of a disk with a radius much greater than its thickness, an infinite slab model will be assumed with losses only out of the two faces.

Let us now divide the infinite medium into two semi-infinite half-spaces with the boundary at $x=0$. Let the right half-space (positive $x$ ) contain a uniform concentration of a gamma emitter while the left half-space (negative $x$ ) contains medium, but no gamma emitter. We wish to calculate the amount of reduction in the volumetric energy absorption rate as a function of position $(x)$ in the right half-space due to gamma leakage across the boundary into the left half-space. Consider the opposite case where the left half-space is filled uniformly with the gamma emitter (same concentration) and the right half-space has none. The energy absorbed at a point $x$ in the right half-space is now just the amount which would be missing if the left half-space were empty as compared to the infinite medium case (i.e., both half-spaces filled with gamma emitter).

This calculation was performed for a series of positive values of $x$ using MICROSHIELD (output files are listed for reference in Attachment 4). A density of $1.5 \mathrm{~g} / \mathrm{cm}^{3}$ was assumed for the medium (both halves) and the gamma emitter was assumed to be ${ }^{137 \mathrm{~m}} \mathrm{Ba}$ at a concentration of $1 \mathrm{Ci} / \mathrm{L}$. The semiinfinite left half-space was simulated by an infinite slab with a thickness of $100 \mathrm{~cm}$. It was found that increasing the slab thickness beyond $100 \mathrm{~cm}$ did not increase the dose rate at a point on the boundary $(x=0)$ and, therefore, the $100 \mathrm{~cm}$ slab was infinitely thick for practical purposes. The results of the calculation for $x$ out to $80 \mathrm{~cm}$ from the boundary are shown in Table 4 . Note that, by superposition, the dose rate for an infinite medium is exactly two times the dose calculated here at the boundary $(x=0)$ between the two halfspaces, or $1.27 \mathrm{E}+3 \mathrm{rads} / \mathrm{h}$. F is the fraction of the infinite medium deposition rate produced by the gamma emitter in the left half-space (i.e., column 2 divided by $1.27 E+3$ rads $/ h)$. The last column $(l-F)$ is the net fraction of the infinite medium deposition rate remaining after the contribution due to the gamma emitter in the left half-space has been subtracted. 
WHC-SD-WM-CN-050 Rev 0

Table 4: Reduction in energy deposition rate due to boundary at $x=0$

\begin{tabular}{cccc}
$\times(\mathrm{cm})$ & $\begin{array}{c}\text { Depos. Rate } \\
(\mathrm{rads} / \mathrm{h})\end{array}$ & $\mathrm{F}$ & $1-\mathrm{F}$ \\
\hline 0 & $6.35 \mathrm{E}+2$ & $5.00 \mathrm{E}-1$ & 0.500 \\
1 & $5.25 \mathrm{E}+2$ & $4.13 \mathrm{E}-1$ & 0.587 \\
10 & $1.54 \mathrm{E}+2$ & $1.21 \mathrm{E}-1$ & 0.879 \\
20 & $4.41 \mathrm{E}+1$ & $3.47 \mathrm{E}-2$ & 0.965 \\
30 & $1.29 \mathrm{E}+1$ & $1.02 \mathrm{E}-2$ & 0.990 \\
40 & $3.83 \mathrm{E}+0$ & $3.02 \mathrm{E}-3$ & 0.997 \\
50 & $1.14 \mathrm{E}+0$ & $8.98 \mathrm{E}-4$ & 0.999 \\
60 & $3.43 \mathrm{E}-1$ & $2.70 \mathrm{E}-4$ & 1.000 \\
70 & $1.03 \mathrm{E}-1$ & $8.11 \mathrm{E}-5$ & 1.000 \\
80 & $3.11 \mathrm{E}-2$ & $2.45 \mathrm{E}-5$ & 1.000
\end{tabular}

Note that the assumed value for gamma emitter concentration is arbitrary and does not effect $F$. Because of the nature of the process the function $F(x)$ can be approximated by an exponential decay function of the form $F=b \exp (-m x)$. Matching the function to the points $F(0 \mathrm{~cm})=0.500$ and $F(10 \mathrm{~cm})=0.121$ in order to obtain the best fit in the region of maximum effect, it is easily shown that $m=0.142 \mathrm{~cm}^{-1}$ and $b=0.500$. This approximation is compared to the numerical results in Table 5.

Table 5: Comparison of $F=0.500 \exp (-0.142 x)$ to numerical results shown in Table 4

\begin{tabular}{ccccc} 
& \multicolumn{2}{c}{ Numerical } & \multicolumn{2}{c}{ Formula } \\
& & $F$ & $F$ & $1-F$ \\
\hline 0 & $5.00 \mathrm{E}-1$ & 0.500 & $5.00 \mathrm{E}-1$ & 0.500 \\
1 & $4.13 \mathrm{E}-1$ & 0.587 & $4.34 \mathrm{E}-1$ & 0.566 \\
10 & $1.21 \mathrm{E}-1$ & 0.879 & $1.21 \mathrm{E}-1$ & 0.879 \\
20 & $3.47 \mathrm{E}-2$ & 0.965 & $2.92 \mathrm{E}-2$ & 0.971 \\
30 & $1.02 \mathrm{E}-2$ & 0.990 & $7.06 \mathrm{E}-3$ & 0.993 \\
40 & $3.02 \mathrm{E}-3$ & 0.997 & $1.71 \mathrm{E}-3$ & 0.998 \\
50 & $8.98 \mathrm{E}-4$ & 0.999 & $4.13 \mathrm{E}-4$ & 1.000 \\
60 & $2.70 \mathrm{E}-4$ & 1.000 & $9.97 \mathrm{E}-5$ & 1.000 \\
70 & $8.11 \mathrm{E}-5$ & 1.000 & $2.41 \mathrm{E}-5$ & 1.000 \\
80 & $2.45 \mathrm{E}-5$ & 1.000 & $5.83 \mathrm{E}-6$ & 1.000
\end{tabular}


In like manner the approximate formula for $F(x)$ can be extended to other isotopes as shown in Table 6 for the principal gamma emitters. The parameter $b=0.500$ in all cases. (corresponding MICROSHIELD run files are listed in Attachment 4.)

Table 6: m parameter values for the principal gamma emitters

\begin{tabular}{llll} 
I sotope & $F(0 \mathrm{~cm})$ & $F(10 \mathrm{~cm})$ & $\mathrm{m}\left(\mathrm{cm}^{-1}\right)$ \\
\hline Co-60 & 0.500 & 0.147 & 0.122 \\
Zr-95 & 0.500 & 0.126 & 0.138 \\
Sb-125 & 0.500 & 0.110 & 0.151 \\
Cs-134 & 0.500 & 0.124 & 0.139 \\
Ba-137m & 0.500 & 0.121 & 0.142 \\
Eu-154 & 0.500 & 0.135 & 0.131
\end{tabular}

The volumetric average energy deposition rate as a fraction of the infinite medium rate in a slab of thickness $T$ in the interval $x=0$ to $x=T$ with the boundary at $x=0$ (i.e., with a boundary on only one side) can be obtained by integrating 1 - F over the slab thickness as follows

$$
\frac{\Phi_{\text {ave }}}{\Phi_{\infty}}=\frac{\int_{0}^{T}(1-F) d x}{T}
$$

or

$$
\frac{\Phi_{\text {ave }}}{\boldsymbol{\Phi}_{\mathrm{\infty}}}=\frac{\int_{0}^{T}\left(1-\frac{1}{2} e^{-m x}\right) d x}{T}
$$

The integration is easily carried out to yield

$$
\frac{\Phi_{\text {ave }}}{\Phi_{\mathrm{a}}}=1-\frac{1}{2 m T}\left[1-e^{-m T}\right]
$$

Note that the fractional average energy deposition rate, $\Phi_{\text {ave }} / \Phi_{\infty}$, goes to 1 as $T$ goes to $\infty$ and to $1 / 2$ as $T$ goes to 0 , as expected. 
Now let us consider the case of a slab of thickness $T$ (from $x=0$ to $x=T$ ) with boundaries on both sides. At position $x$ (where $0 \leq x \leq T$ ) the total fractional loss due to absence of active material outside the slab is therefore equal to $F_{1}+F_{2}$ where, by symmetry,

$$
F_{1}=\frac{1}{2} e^{-m x}
$$

and

$$
F_{2}=\frac{1}{2} e^{-m(T-x)}
$$

The average fractional energy deposition rate is, as before,

$$
\frac{\boldsymbol{\Phi}_{\text {ave }}}{\boldsymbol{\Phi}_{\infty}}=\frac{\int_{0}^{T}\left(1-F_{1}-F_{2}\right) d x}{T}
$$

where, using eqns. 4 an 5 ,

$$
1-F_{1}-F_{2}=1-\frac{1}{2}\left(e^{-m x}-e^{m x} e^{-m T}\right)
$$

Again the integration is easily carried out to yield

$$
\frac{\Phi_{\text {ave }}}{\Phi_{\infty}}=1-\frac{1}{m T},\left(1-e^{-m T}\right)
$$

As before, the fractional average energy deposition rate goes to 1 as $T$ goes to $\infty$, however in this case the energy deposition goes to 0 as $T$ goes to 0 since the active material disappears. Typical values of the correction to be made for gamma leakage in a slab geometry (double boundary) are shown in Table 7 for the cases of ${ }^{137 \mathrm{~m}} \mathrm{Ba}(0.66 \mathrm{MeV} r)$ and ${ }^{154} \mathrm{Eu}(1.3 \mathrm{MeV} v)$. This correction factor can be applied directly to an internal gamma dose rate calculated with the infinite medium assumption (such as the ones reported in column 4 of Table 2). 
WHC-SD-WM-CN-050 Rev 0

Table 7: Gamma leakage correction factors for ${ }^{137 \mathrm{~m}} \mathrm{Ba}$
$\left(\mathrm{m}=0.142 \mathrm{~cm}^{-1}\right)$ and ${ }^{154} \mathrm{Eu}\left(\mathrm{m}=0.131 \mathrm{~cm}^{-1}\right.$ )
in a slab geometry (double boundary)

\begin{tabular}{ccc}
$T(\mathrm{~cm})$ & ${ }^{137 \mathrm{~m}_{\mathrm{Ba}} \Phi_{\mathrm{ave}} / \Phi_{\mathrm{w}}{ }^{154} \mathrm{Eu}}$ \\
\hline 0 & 0 & 0 \\
1 & 0.068 & 0.062 \\
5 & 0.284 & 0.266 \\
10 & 0.466 & 0.443 \\
20 & 0.668 & 0.646 \\
30 & 0.769 & 0.751 \\
40 & 0.825 & 0.810 \\
50 & 0.859 & 0.848 \\
100 & 0.930 & 0.924 \\
500 & 0.986 & 0.985 \\
1000 & 0.993 & 0.992
\end{tabular}

It should be noted that these factors only correct for gamma leakage from the faces of an infinite slab. They do not account for the leakage which would occur from the edges of a finite slab (such as a disk). For the cases of interest here, however, the source disk diameter is over $2000 \mathrm{~cm}$ so the correction for edge leakage would be negligible. In addition, if a substantial layer of other material lies under (or on top of) the supernate layer with about the same gamma activity as the supernate, then only gamma leakage from one surface of the supernate layer should be corrected for. The single boundary correction factor given by Eqn.3 will work nicely for this. Note that the reduction in the average energy deposition rate for such a case will be exactly half the reduction for the double boundary case given by Eqn.8.

If a mix of radionuclides is present, the dose rates could be calculated and corrected separately and added, or, alternatively, the correction factor corresponding to the nuclide with the lowest energy gammas (least leakage) could be applied to the total dose rate. A lower energy gamma corresponds to a higher attenuation factor, i.e., a higher value of $m$ in Table 6 . The correction factor for ${ }^{137 \mathrm{~m}} \mathrm{Ba}\left(\mathrm{m}=0.142 \mathrm{~cm}^{-1}\right)$ could therefore be conservatively applied to a ${ }^{137 \mathrm{~m}} \mathrm{Ba}+{ }^{154} \mathrm{Eu}$ mix.

Correction factors for gamma leakage which could be applied to the gamma energy deposition rates shown in column 4 of Table 2 are shown in Table 8 . These factors are conservatively based on leakage due to ${ }^{137 \mathrm{~m}} \mathrm{Ba}$ gammas $(\mathrm{m}=$ $\left.0.142 \mathrm{~cm}^{-1}\right)$. 


$$
\text { WHC-SD-WM-CN-050 Rev } 0
$$

Table 8: Gamma leakage correction factors for tank mix cases shown in Table $2\left(\mathrm{~m}=0.142 \mathrm{~cm}^{-1}\right)$

\begin{tabular}{|c|c|c|c|}
\hline Tank Mix & $\begin{array}{l}\text { Supernate } \\
\text { Thickness }(\mathrm{cm})\end{array}$ & $\begin{array}{l}\text { Leakage } \\
\text { Single } \\
\text { Boundary }\end{array}$ & $\begin{array}{c}\text { Correction } \\
\text { Double } \\
\text { Boundary }\end{array}$ \\
\hline $105 \mathrm{AP}$ & 18.5 & 0.823 & 0.647 \\
\hline $103 C+105 A P$ & 141 & 0.975 & 0.950 \\
\hline $107 \mathrm{AP}$ & 28.5 & 0.879 & 0.758 \\
\hline $103 \mathrm{C}+107 \mathrm{AP}$ & 151 & 0.977 & 0.953 \\
\hline $\begin{array}{l}103 \mathrm{U}+105 \mathrm{U}+ \\
109 \mathrm{U}+107 \mathrm{AP}\end{array}$ & 475 & 0.993 & 0.985 \\
\hline $\begin{array}{l}103 C+103 U_{+} \\
105 U_{+} 109 U_{+} \\
107 \mathrm{AP}\end{array}$ & 598 & 0.994 & 0.988 \\
\hline $102 \mathrm{AY}$ & 729 & 0.995 & 0.990 \\
\hline $103 \mathrm{C}+102 \mathrm{AY}$ & 850 & 0.996 & 0.992 \\
\hline $102 A Z$ & 652 & 0.995 & 0.989 \\
\hline
\end{tabular}

\section{Conclusion:}

Independent calculations of the total energy deposition rates in the dispersed supernate/organic mixes agreed well as shown in Table 2. Calculations of energy deposition rates in the organic layer cases tracked reasonably well as shown in Table 3, although the agreement was not as good as for the dispersed cases. A scheme for correcting for gamma leakage was developed, but for most cases the correction is small. The only appreciable correction in the cases shown in Table 2 is for I05AP where the total average energy deposition rate would be reduced by $17 \%$. This is the only case considered here where the supernate/organic layer was relatively thin and the gamma activity contributed a major fraction of the overall heating.

\section{References:}

Grove 1988, Microshield 3 Manual, Grove Engineering, Inc., Apri1 12, 1988. 


\section{WHC-SD-WM-CN-050 Rev 0}

\section{Attachment 1 \\ DST Energy Deposition Rate Calculations \\ to be Verified}




\begin{tabular}{|c|c|c|c|c|c|c|}
\hline \multirow{3}{*}{$4 / 22 / 96$} & \multicolumn{6}{|c|}{ Energy deposition in dispersed and layered organic } \\
\hline & \multicolumn{6}{|c|}{ within DSTs is given below. Dispersed case assumes no } \\
\hline & \multicolumn{5}{|c|}{ alpha, beta or gamma losses from the supernate. } & \\
\hline & \multicolumn{5}{|c|}{ The layered case assumes a constant dose rate } & \\
\hline & \multicolumn{6}{|c|}{ equal to the gamma dose rate $5 \mathrm{~cm}$ into the organic, } \\
\hline & \multicolumn{4}{|c|}{ as calculated by MICROSHIELD. } & & \\
\hline & \multicolumn{4}{|c|}{ SUPERNATE CURIES PER LITER } & & \\
\hline \multicolumn{7}{|c|}{\begin{tabular}{|l|l|l|l|} 
FOR THE DISPERSED ORGANIC CASE: & & & \\
\end{tabular}} \\
\hline ISOTOPES & $101 \mathrm{AN}$ & 102AN & 103AN & 104AN & $105 \mathrm{AN}$ & TOGAN \\
\hline $14 \mathrm{C}$ & $3.23 \mathrm{E}-08$ & 1.74E-05 & $1.96 \bar{E}-06$ & $0.00 E+00$ & $0.005+00$ & 4.52E-08 \\
\hline $90 \mathrm{~s} r$ & $1.90 \mathrm{E}-05$ & $5.48 E-02$ & $7.13 \mathrm{E}-03$ & $4.86 E-03$ & $1.59 \mathrm{E}-03$ & $6.32 \mathrm{E}-03$ \\
\hline $90 Y$ & $1.90 E-05$ & $5.48 \mathrm{E}-02$ & $7.13 \mathrm{E}-03$ & $4.86 \mathrm{E}-03$ & $1.59 \mathrm{E}-03$ & 6.32E-03 \\
\hline 997c & $9.82 E-06$ & $2.95 \mathrm{E}-04$ & $1.67 \mathrm{E}-04$ & 1.80E-04 & $1.75 \mathrm{E}-04$ & 6.41 E-0 \\
\hline $137 \mathrm{Cs}$ & $2.64 \mathrm{E}-06$ & $1.82 \mathrm{E}-01$ & $4.24 \mathrm{E}-01$ & $3.86 \mathrm{E}-01$ & $2.57 E-01$ & $3.53 \mathrm{E}-02$ \\
\hline $137 \mathrm{Ba}$ & $2.51 \mathrm{E}-06$ & 1.73E-01 & $4.02 \mathrm{E}-01$ & 3.67E-01 & $2.44 \mathrm{E}-01$ & $3.36 \mathrm{E}-02$ \\
\hline 154Eu & $1.76 \mathrm{E}-06$ & $7.58 \mathrm{E}-04$ & $0.00 \mathrm{E}+00$ & $0.00 E+00$ & $0.00 E+00$ & $1.80 \mathrm{E}-06$ \\
\hline $235 \mathrm{U}$ & $0.00 \mathrm{E}+00$ & $0.00 \mathrm{E}+00$ & $0.00 E+00$ & $0.00 E+00$ & $0.00 E+00$ & $0.00 \mathrm{E}+00$ \\
\hline 2380 & $0.00 \mathrm{E}+00$ & $0.00 \mathrm{E}+00$ & $0.00 \bar{E}+00$ & $0.00 E+00$ & $0.00 \mathrm{E}+00$ & $0.00 E+00$ \\
\hline $237 \mathrm{~Np}$ & $0.00 \mathrm{E}+00$ & $0.00 \mathrm{E}+00$ & $0.00 \mathrm{E}+00$ & $0.00 \mathrm{E}+00$ & $0.00 \mathrm{E}+00$ & $4.69 \mathrm{E}-10$ \\
\hline $238 \mathrm{Pu}$ & $0.00 E+00$ & $0.00 E+00$ & $0.00 \mathrm{E}+00$ & $0.00 \mathrm{E}+00$ & $0.00 E+00$ & $4.30 \mathrm{E}-07$ \\
\hline 239Pu & $8.57 \bar{c}-09$ & $6.37 \mathrm{E}-05$ & $1.87 \bar{E}-06$ & $1.04 E-05$ & $1.04 \mathrm{E}-05$ & $2.99 \mathrm{E}-06$ \\
\hline $240 \mathrm{Pu}$ & $8.58 \mathrm{E}-12$ & $1.62 \mathrm{E}-05$ & 2.87E-07 & $1.60 E-06$ & 1.59E-06 & $7.38 \mathrm{E}-07$ \\
\hline 241Pu & $0.00 \mathrm{E}+00$ & $1.56 \mathrm{E}-05$ & $3.62 \mathrm{E}-07$ & $1.59 \mathrm{E}-06$ & 1.58E-06 & $2.30 \mathrm{E}-07$ \\
\hline $241 \mathrm{Am}$ & 1.65 E- 07 & $5.91 \mathrm{E}-05$ & 2.24E-06 & 1.52E-06 & $1.51 \mathrm{E}-06$ & 1.62E-05 \\
\hline SUPERNATE VOLUME (LITERS) & $3.68 \mathrm{E}+06$ & $3.95 \mathrm{E}+06$ & $3.62 E+06$ & $3.02 E+06$ & $4.29 E+06$ & $4.47 E+06$ \\
\hline SUPERNATE DENSITY (GRAMS/CC) & 1.3 & 1.37 & 1.5 & 1.46 & 1.46 & 1.37 \\
\hline SUPERNATE THICKNESS (CM) & $8.98 \mathrm{E}+02$ & $9.65 \mathrm{E}+02$ & $8.84 E+02$ & $7.38 E+02$ & $1.05 \mathrm{E}+03$ & $1.09 E+03$ \\
\hline SUPERNATE DIAMETER (CM) & 2286 & 2286 & 2286 & 2286 & 2286 & 2286 \\
\hline ALPHA ERGS PER CC-HR & $2.03 \mathrm{E}-01$ & $1.57 \mathrm{E}+02$ & $4.99 \mathrm{E}+00$ & $1.50 E+01$ & $1.49 E+01$ & $2.36 \mathrm{E}+01$ \\
\hline BETA ERGS PER CC-HR & $4.76 E+00$ & $1.99 E+04$ & $1.72 \mathrm{E}+04$ & $1.53 E+04$ & $9.75 E+03$ & $2.81 E+03$ \\
\hline GAMMA ERGS PER CC-HR & $6.20 \mathrm{E}-01$ & $2.45 E+04$ & $5.68 E+04$ & $5.18 E+04$ & $3.44 \overline{E+04}$ & $4.73 \mathrm{E}+03$ \\
\hline SUPERNATE/ORGANIC RAD/HR & $4.30 \mathrm{E}-02$ & $3.25 \mathrm{E}+02$ & $4.93 E+02$ & $4.59 E+02$ & $3.03 \mathrm{E}+02$ & $5.53 E+01$ \\
\hline GAMMA ONLY RAD/HR & $4.77 E-03$ & $1.79 E+02$ & $3.78 E+02$ & $3.55 E+02$ & $2.36 \mathrm{E}+02$ & $3.46 \mathrm{E}+0$ \\
\hline \multicolumn{7}{|l|}{ FOR THE LAYERED ORGANIC CASE: } \\
\hline SUPERNATE Ba-137 CURIES & $9.21 E+00$ & $6.83 E+05$ & $1.46 E+06$ & $1.11 \mathrm{E}+06$ & $1.05 E+06$ & $1.50 \bar{E}+05$ \\
\hline SUPERNATE EU-154 CURIES & $6.46 \mathrm{E}+00$ & $3.00 \mathrm{E}+03$ & $0.00 E+00$ & $0.00 E+00$ & $0.00 E+00$ & $8.03 E+00$ \\
\hline RADIHR TO ORGANIC LAYER & & & & & & \\
\hline
\end{tabular}




\begin{tabular}{|c|c|c|c|c|c|c|}
\hline \multicolumn{7}{|l|}{$4 / 22 / 96$} \\
\hline & & & & & & \\
\hline & & & & & & \\
\hline & & & & & & \\
\hline & & & & & & \\
\hline & & & & & & \\
\hline & \multicolumn{4}{|c|}{ SUPERNATE CURIES PER LITER } & & \\
\hline \multicolumn{7}{|l|}{ FOR THE DISPERSED ORGANIC CASE: } \\
\hline ISOTOPES & 107AN & $101 \mathrm{AP}$ & $102 \mathrm{AP}$ & 103AP & 104AP & 105AP $=$ \\
\hline $14 \mathrm{C}$ & $0.00 E+00$ & $1.96 \mathrm{E}-07$ & $4.19 \mathrm{E}-07$ & $4.46 \mathrm{E}-09$ & $2.52 \mathrm{E}-14$ & $8.73 E-09$ \\
\hline $90 \mathrm{Sr}$ & 4.83E-02 & $1.14 E-04$ & $8.71 E=04$ & $1.53 \mathrm{E}-06$ & $2.16 \mathrm{E}-09$ & $5.72 E-03$ \\
\hline soY & 4.83E-02 & 1.14E-04 & $8.71 E-04$ & $1.53 \mathrm{E}-06$ & $2.16 \mathrm{E}-09$ & $5.72 \mathrm{E}-03$ \\
\hline 99Tc & $4.74 E-04$ & 6.31E-05 & $8.58 \mathrm{E}-05$ & $1.08 \mathrm{E}-06$ & 1.99E-07 & 4.46E-05 \\
\hline $137 \mathrm{Cs}$ & $1.38 E-01$ & $1.28 \mathrm{E}-01$ & $1.42 \mathrm{E}-03$ & $3.92 \mathrm{E}-03$ & $1.29 E-05$ & $1.76 \mathrm{E}-02$ \\
\hline $137 \mathrm{Ba}$ & 1.31E-01 & $1.21 \mathrm{E}-01$ & $1.34 E-03$ & $3.72 \mathrm{E}-03$ & $1.23 \mathrm{E}-05$ & 1.67E-02 \\
\hline 154EU & $1.45 \mathrm{E}-03$ & 4.94E-09 & $0.00 \mathrm{E}+00$ & $1.69 E-05$ & $0.00 \mathrm{E}+00$ & $0.00 E+00$ \\
\hline $235 \mathrm{U}$ & $0.00 \mathrm{E}+00$ & $0.00 \mathrm{E}+00$ & $0.00 E+00$ & $0.00 \mathrm{E}+00$ & $0.00 \mathrm{E}+00$ & $0.00 \mathrm{E}+00$ \\
\hline 2380 & $0.00 E+00$ & $0.00 E+00$ & $0.00 \mathrm{E}+00$ & $0.00 E+00$ & $0.00 E+00$ & $0.00 E+00$ \\
\hline $237 \mathrm{~Np}$ & $0.00 E+00$ & $0.00 \mathrm{E}+00$ & $0.00 \mathrm{E}+00$ & $0.00 \mathrm{E}+00$ & $5.36 \mathrm{E}-10$ & $0.00 E+00$ \\
\hline $238 \mathrm{Pu}$ & $0.00 \mathrm{E}+00$ & $0.00 \mathrm{E}+00$ & $0.00 \mathrm{E}+00$ & $0.00 \mathrm{E}+00$ & $0.00 \mathrm{E}+00$ & $0.00 \mathrm{E}+00$ \\
\hline $239 \mathrm{Pu}$ & $3.55 \mathrm{E}-05$ & $1.44 \mathrm{E}-07$ & $7.50 \mathrm{E}-08$ & 6.97E-09 & $4.46 \mathrm{E}-10$ & 2.41E-06 \\
\hline $240 \mathrm{Pu}$ & $9.03 \mathrm{E}-06$ & $2.22 \mathrm{E}-08$ & $1.15 \mathrm{E}-08$ & 1.07E-09 & $1.61 \mathrm{E}-10$ & $6.02 \mathrm{E}-07$ \\
\hline $241 \mathrm{Pu}$ & $8.52 \mathrm{E}-06$ & $3.30 \mathrm{E}-08$ & 1.70E-08 & 1.47E-09 & $1.56 \mathrm{E}-09$ & $2.14 E-07$ \\
\hline $241 \mathrm{Am}$ & 3.50 E-04 & 3.67E-07 & 4.17E-07 & $1.13 \mathrm{E}-08$ & $1.50 \mathrm{E}-12$ & $1.34 E-05$ \\
\hline SUPERNATE VOLUME (LITERS) & $3.68 \mathrm{E}+06$ & $3.39 \mathrm{E}+06$ & $4.16 E+06$ & $1.02 \mathrm{E}+05$ & $4.23 \mathrm{E}+06$ & $7.56 \mathrm{E}+04$ \\
\hline SUPERNATE DENSITY (GRAMS/CC) & 1.33 & 1.32 & 1.21 & 1.01 & 1.28 & 1.32 \\
\hline SUPERNATE THICKNESS (CM) & $8.99 \mathrm{E}+02$ & $8.27 \mathrm{E}+02$ & $1.02 E+03$ & $2.50 \mathrm{E}+01$ & $1.03 E+03$ & $1.85 \mathrm{E}+01$ \\
\hline SUPERNATE DIAMETER (CM) & 2286 & 2286 & 2286 & 2286 & 2286 & 2286 \\
\hline ALPHA ERGS PER CC-HR & $4.59 \mathrm{E}+02$ & $6.13 E-01$ & 5.83E-01 & $2.21 E-02$ & $1.22 \mathrm{E}-03$ & $1.90 \mathrm{E}+01$ \\
\hline BETA ERGS PER CC.HR & 1.67E+04 & $4.68 \mathrm{E}+03$ & $2.62 E+02$ & $1.44 \mathrm{E}+02$ & $4.72 \mathrm{E}-01$ & $2.02 \mathrm{E}+03$ \\
\hline GAMMA ERGS PER CC-HR & $1.87 E+04$ & $1.71 E+04$ & $1.90 \mathrm{E}+02$ & $5.28 \mathrm{E}+02$ & $1.73 \mathrm{E}+00$ & $2.35 \mathrm{E}+03$ \\
\hline SUPERNATE/ORGANIC RAD/HR & $2.70 \mathrm{E}+02$ & $1.65 \mathrm{E}+02$ & $3.74 \mathrm{E}+00$ & $6.65 E+00$ & $1.72 \mathrm{E}-02$ & $3.33 E+01$ \\
\hline GAMMA ONLY RAD/HR & $1.41 \mathrm{E}+02$ & $1.30 \mathrm{E}+02$ & $1.57 \mathrm{E}+00$ & $5.22 \mathrm{E}+00$ & $1.35 \mathrm{E}-02$ & $1.78 E+01$ \\
\hline \multirow{2}{*}{\multicolumn{7}{|c|}{ FOR THE LAYERED ORGANIC CASE: }} \\
\hline & & & & & & \\
\hline SUPERNATE Ba-137 CURIES & $4.82 E+05$ & $4.11 \mathrm{E}+05$ & $5.59 E+03$ & $3.80 \mathrm{E}+02$ & $5.20 \mathrm{E}+01$ & $1.26 E+03$ \\
\hline SUPERNATE EU-154 CURIES & $5.33 \mathrm{E}+03$ & $1.67 \overline{\mathrm{E}}-02$ & $0.00 E+00$ & $1.73 E+00$ & $0.00 \mathrm{E}+00$ & $0.00 \mathrm{E}+00$ \\
\hline RAD/HR TO ORGANIC LAYER & & & & & & 6.18 \\
\hline
\end{tabular}




\begin{tabular}{|c|c|c|c|c|c|}
\hline \multicolumn{6}{|l|}{$4 / 22 / 96$} \\
\hline & & & & & \\
\hline & & & & & \\
\hline & & & & & \\
\hline & & & & & \\
\hline & & & & & \\
\hline & \multicolumn{4}{|c|}{ SUPERNATE CURIES PER LITER } & \\
\hline FOR THE DISPERSED ORGANIC CASE: & $103 \mathrm{C}+$ & $106 \mathrm{AP}$ & & $5103 \mathrm{C}+4$ & $103 U+105 U+1094+$ \\
\hline ISOTOPES & T105AP & & 107AP & 107AP $=$ & B07AP \\
\hline $14 C$ & & $4.09 \bar{E}-14$ & 8.97E-11 & & \\
\hline $90 \mathrm{sr}$ & $9.64 E-04$ & $3.19 \mathrm{E}-10$ & 2.02E-09 & $2.03 E-04$ & $9.14 \mathrm{E}-03$ \\
\hline soY & $9.64 E-04$ & $3.19 E-10$ & 2.02E-09 & $2.03 E-04$ & $9.14 \mathrm{E}-03$ \\
\hline $99 \mathrm{TC}$ & & $2.93 \mathrm{E}-11$ & $5.78 \mathrm{E}-10$ & & \\
\hline $137 \mathrm{Cs}$ & 5.79E-02 & $4.75 \mathrm{E}-08$ & 8.77E-09 & $5.19 \mathrm{E}-02$ & $3.33 \mathrm{E}-01$ \\
\hline $137 \mathrm{Ba}$ & $5.50 \mathrm{E}-02$ & $4.51 E-08$ & $8.33 E-09$ & $4.93 \mathrm{E}-02$ & $3.16 E-01$ \\
\hline 15AEU & & $2.22 E-12$ & 3.27E-09 & & \\
\hline 2350 & & $0.00 E+00$ & $0.00 \mathrm{E}+00$ & & \\
\hline $238 \mathrm{U}$ & & $0.00 \mathrm{E}+00$ & $0.00 \mathrm{E}+00$ & & \\
\hline $237 \mathrm{~Np}$ & & $4.54 \mathrm{E}-14$ & $0.00 \mathrm{E}+00$ & & \\
\hline $238 \mathrm{Pu}$ & & $0.00 E+00$ & $1.53 \mathrm{E}-11$ & & \\
\hline $239 \mathrm{Pu}$ & & $5.36 \mathrm{E}-12$ & $1.51 E-08$ & & \\
\hline $240 \mathrm{Pu}$ & & $2.81 \mathrm{E}-14$ & 2.31E-00 & & \\
\hline $241 P u$ & & $1.87 \mathrm{E}-13$ & 3.45E-09 & & \\
\hline $241 \mathrm{Am}$ & & $1.61 \mathrm{E}-11$ & $1.78 \mathrm{E}-08$ & & \\
\hline SUPERNATE VOLUME (LITERS) & $5.79 E+05$ & $3.03 E+06$ & 1.17E+05 & $6.20 E+05$ & $1.95 \mathrm{E}+06$ \\
\hline SUPERNATE DENSITY (GRAMS/CC) & 1.10 & 1.38 & 1.01 & 1.06 & 1.35 \\
\hline SUPERNATE THICKNESS (CM) & $1.44 \mathrm{E}+02$ & $7.40 \mathrm{E}+02$ & $2.86 \mathrm{E}+01$ & $1 . \overline{51 E+02}$ & $4.75 \mathrm{E}+02$ \\
\hline SUPERNATE DIAMETER (CM) & 2286 & 2286 & 2286 & 2286 & 2286 \\
\hline ALPHA ERGS PER CC-HR & & $2.48 \mathrm{E}-05$ & $4.00 \mathrm{E}-02$ & & \\
\hline BETA ERGS PER CC. HR & $2.34 \mathrm{E}+03$ & $1.81 E-03$ & $9.66 \mathrm{E}-04$ & $1.94 \mathrm{E}+03$ & $1.43 \mathrm{E}+04$ \\
\hline GAMMA ERGS PER CC-HR & $7.76 \mathrm{E}+03$ & $6.36 E-03$ & $1.67 E-03$ & $6.96 \mathrm{E}+03$ & $4.46 E+04$ \\
\hline SUPERNATE/ORGANIC RAD/HR & $9.17 \mathrm{E}+01$ & $5.94 \mathrm{E}-05$ & 4.22E-04 & $8.40 E+01$ & $4.38 E+02$ \\
\hline GAMMA ONLY RAD/HR & $7.04 \bar{E}-01$ & 4.61E-05 & 1.65E-05 & 6.57E+01 & $3.31 \mathrm{E}+02$ \\
\hline FOR THE LAYERED ORGANIC CASE: & & & & & \\
\hline SUPERNATE Ba-137 CURIES & $3.19 E+04$ & 1.37E-01 & $9.77 \bar{E}-04$ & $3.06 \mathrm{E}+04$ & $6.14 \mathrm{E}+05$ \\
\hline SUPERNATE EU-154 CURIES & & $6.73 E-06$ & $3.84 E-04$ & & \\
\hline RAD/HR TO ORGANIC LAYER & $27 . \overline{8}$ & & $4.46 \mathrm{E}-06$ & 25.83 & 133.6 \\
\hline
\end{tabular}




\begin{tabular}{|c|c|c|c|c|c|}
\hline \multicolumn{6}{|l|}{$4 / 22 / 96$} \\
\hline & & & & & \\
\hline & & & & & \\
\hline & & & & & \\
\hline & & & & & \\
\hline & \multicolumn{3}{|c|}{ SUPERNATE CURIES PER LITER } & & \\
\hline FOR THE DISPERSED ORGANIC CASE: & 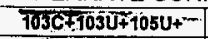 & & & & \\
\hline ISOTOPES & BOSU+107AP & 108AP & 101AW & 102AW & 103AW \\
\hline $14 C$ & & $2.99 \mathrm{E}-12$ & $3.69 \mathrm{E}-07$ & & $0.00 E+00$ \\
\hline $90 \mathrm{ST}$ & $7.31 \mathrm{E}-03$ & $1.80 E-04$ & $5.63 E-04$ & & $0.00 E+00$ \\
\hline $90 Y$ & $7.31 E-03$ & 1.80E-04 & $5.63 E-04$ & & $0.00 E+00$ \\
\hline $99 \mathrm{Tc}$ & & $1.54 E-04$ & $1.51 E-04$ & & $0.00 E+00$ \\
\hline $137 \mathrm{Cs}$ & 2.77E-01 & $1.10 \mathrm{E}-01$ & $2.68 \mathrm{E}-01$ & & 4.75E-02 \\
\hline $137 \mathrm{Ba}$ & 2.63E-01 & $1.05 \mathrm{E}-01$ & 2.55E-01 & & $4.51 \mathrm{E}-02$ \\
\hline 154Eu & & 1.63E-09 & $0.00 E+00$ & & $0.00 E+00$ \\
\hline $235 \mathrm{U}$ & & $0.00 E+00$ & $0.00 E+00$ & & $0.00 E+00$ \\
\hline $238 \mathrm{U}$ & & $0.00 \mathrm{E}+00$ & $0.00 \mathrm{E}+00$ & & $0.00 \mathrm{E}+00$ \\
\hline $237 \mathrm{~Np}$ & & 1.15E-11 & $0.00 E+00$ & & $0.00 E+00$ \\
\hline $238 \mathrm{Pu}$ & & $1.05 \mathrm{E}-08$ & $0.00 E+00$ & & $1.16 \mathrm{E}-04$ \\
\hline 239Pu & & $6.93 \mathrm{E}-12$ & $1.54 \mathrm{E}-06$ & & $0.00 E+00$ \\
\hline $240 \mathrm{Pu}$ & & $1.06 \mathrm{E}-12$ & $2.36 E-07$ & & $0.00 \mathrm{E}+00$ \\
\hline 241Pu & & $1.75 E-13$ & $3.04 E-07$ & & $0.00 \mathrm{E}+00$ \\
\hline $241 \mathrm{Am}$ & & $1.55 E-12$ & 1.61E-06 & & $0.00 \mathrm{E}+00$ \\
\hline SUPERNATE VOLUME (LITERS) & $2.45 E+06$ & $3.10 \mathrm{E}+06$ & $3.94 E+06$ & & $5.41 \mathrm{E}+05$ \\
\hline SUPERNATE DENSITY (GRAMS/CC) & 1.29 & 1.1 & 1.51 & 0 & 1.04 \\
\hline SUPERNATE THICKNESS (CM) & $5.98 \mathrm{E}+02$ & $7.57 \bar{E}+02$ & $9.62 \mathrm{E}+02$ & $0.00 E+00$ & $1.32 \mathrm{E}+02$ \\
\hline SUPERNATE DIAMETER (CM) & 2286 & 2286 & 2286 & 2286 & 2286 \\
\hline ALPHA ERGS PER CC-HR & & $1.24 E-02$ & $3.84 \mathrm{E}+00$ & $0.00 \mathrm{E}+00$ & $1.36 \mathrm{E}+02$ \\
\hline BETA ERGS PER CC-HR & $1.19 E+04$ & $4.06 E+03$ & $9.92 \mathrm{E}+03$ & $0.00 E+00$ & $1.73 E+03$ \\
\hline GAMMA ERGS PER CC-HR & $3.72 E+04$ & $1.48 E+04$ & $3.60 E+04$ & $0.00 \mathrm{E}+00$ & $6.37 \mathrm{E}+03$ \\
\hline SUPERNATE/ORGANIC RAD/HR & $3.80 \mathrm{E}+02$ & $1.71 \mathrm{E}+02$ & $3.04 E+02$ & $0.00 E+00$ & $7.92 \mathrm{E}+01$ \\
\hline GAMMA ONLY RAD/HR & $2.88 \mathrm{E}+02$ & $1.34 \mathrm{E}+02$ & $2.38 \mathrm{E}+02$ & & $6.12 \mathrm{E}+01$ \\
\hline FOR THE LAYERED ORGANIC CASE: & & & & & \\
\hline SUPERNATE Ba-137 CURIES & $6.45 E+05$ & $3.25 E+05$ & $1.00 E+06$ & $0.00 \mathrm{E}+00$ & $2.44 E+04$ \\
\hline SUPERNATE EU-154 CURIES & & $5.05 \mathrm{E}-03$ & $0.00 E+00$ & $0.00 \mathrm{E}+00$ & $0.00 E+00$ \\
\hline RAD/HR TO ORGANIC LAYER & 131.3 & & & & \\
\hline
\end{tabular}


ENERGY DEPOSITION IN DST

\begin{tabular}{|c|c|c|c|c|c|c|}
\hline \multicolumn{7}{|l|}{$4 / 22 / 96$} \\
\hline & & & & & & \\
\hline & & & & & & \\
\hline & & & & & & \\
\hline & & & & & & \\
\hline & \multirow{2}{*}{\multicolumn{4}{|c|}{ SUPERNATE CURIES PER LITER }} & & \\
\hline FOR THE DISPERSED ORGANIC CASE: & & & & & & \\
\hline$\frac{\text { FUR IHE DISPERSED URGANIC CASE: }}{\text { ISOTOPES }}$ & & & & & & $103 c+\cdots$ \\
\hline ISOTOPES & 104AW & 105AW & 106AW & $101 \mathrm{AY}$ & -102AY & 102AY \\
\hline \begin{tabular}{|l|}
$14 C$ \\
$90 \mathrm{Sr}$
\end{tabular} & 4.48E-08 & $5.04 \mathrm{E}-15$ & & $1.89 \mathrm{E}-06$ & $0.00 E+00$ & \\
\hline \begin{tabular}{|l|}
$0 \mathrm{Sr}$ \\
$90 \mathrm{Y}$
\end{tabular} & $6.99 \mathrm{E}-05$ & 3.77E-11 & & $3.18 \mathrm{E}-02$ & $1.37 E-03$ & $1.21 E-03$ \\
\hline \begin{tabular}{|l} 
90Y \\
99Tc \\
\end{tabular} & $6.99 E-05$ & 3.77E-11 & & $3.18 \mathrm{E}-02$ & $1.37 E-03$ & 1.21E-03 \\
\hline & $1.36 \mathrm{E}-05$ & $3.61 \mathrm{E}-12$ & & $9.10 \mathrm{E}-05$ & 2.24E-05 & \\
\hline & $1.40 \mathrm{E}-02$ & $2.93 \mathrm{E}-04$ & & 1.25E-01 & $4.87 \mathrm{E}-02$ & $5.09 E-02$ \\
\hline & 1.33E-02 & $2.78 \mathrm{E}-04$ & & 1.19E-01 & 4.62E-02 & 4.83E-02 \\
\hline & 2.44E-06 & $2.74 \mathrm{E}-13$ & & $0.00 \mathrm{E}+00$ & $0.00 E+00$ & \\
\hline $235 \mathrm{U}$ & $0.00 \mathrm{E}+00$ & $0.00 \mathrm{E}+00$ & & $0.00 \mathrm{E}+00$ & $0.00 \mathrm{E}+00$ & \\
\hline $238 \mathrm{U}$ & $0.00 \mathrm{E}+00$ & $0.00 \mathrm{E}+00$ & & $0.00 \mathrm{E}+00$ & $0.00 E+00$ & \\
\hline $237 N p$ & $0.00 \mathrm{E}+00$ & $1.53 \mathrm{E}-10$ & & 1.69E-07 & $0.00 \mathrm{E}+00$ & \\
\hline 238Pu & $0.00 E+00$ & $1.40 \mathrm{E}-07$ & & $0.00 \mathrm{E}+00$ & $0.00 \mathrm{E}+00$ & \\
\hline $239 \mathrm{Pu}$ & $6.87 E-08$ & $6.61 E-13$ & & 9.39E-08 & $2.06 \mathrm{E}-05$ & \\
\hline $240 \mathrm{Pu}$ & $9.87 \mathrm{E}-09$ & 3.47E-15 & & $2.57 \mathrm{E}-08$ & $0.00 \mathrm{E}+00$ & \\
\hline $241 \mathrm{Pu}$ & $1.55 \mathrm{E}-08$ & $2.13 \mathrm{E}-14$ & & $5.89 \mathrm{E}-08$ & $0.00 E+00$ & \\
\hline 241Am & 1.57E-07 & $1.98 \mathrm{E}-12$ & & $1.52 \mathrm{E}-04$ & 1.44E-07 & \\
\hline SUPERNATE VOLUME (LITERS) & $5.68 \mathrm{E}+05$ & $4.31 E+06$ & & $1.52 \mathrm{E}+06$ & $2.98 \mathrm{E}+06$ & $3.48 \mathrm{E}+06$ \\
\hline SUPERNATE DENSITY (GRAMS/CC) & 1.07 & 1.06 & 0 & 1.05 & 1.14 & 1.13 \\
\hline SUPERNATE THICKNESS (CM) & $1.39 \mathrm{E}+02$ & $1.05 \mathrm{E}+03$ & $0.00 \mathrm{E}+00$ & $3.70 E+02$ & $7.29 \mathrm{E}+02$ & $8.50 E+02$ \\
\hline SUPERNATE DIAMETER (CM) & 2286 & 2286 & 2286 & 2286 & 2286 & 2286 \\
\hline ALPHA ERGS PER CC-HR & $2.70 E-01$ & 1.65E-01 & $0.00 E+00$ & $1.79 \mathrm{E}+02$ & $2.29 \mathrm{E}+01$ & \\
\hline BETA ERGS PER CC-HR & $5.27 \mathrm{E}+02$ & $1.07 E+01$ & $0.00 \bar{E}+00$ & $1.23 \mathrm{E}+04$ & $2.11 \mathrm{E}+03$ & 2.15E+03 \\
\hline GAMMA ERGS PER CC-HR & $1.87 \mathrm{E}+03$ & $3.93 E+01$ & $0.00 \mathrm{E}+00$ & $1.68 \mathrm{E}+04$ & $6.52 \mathrm{E}+03$ & $6.82 \mathrm{E}+03$ \\
\hline SUPERNATE/ORGANIC RAD/HR & $2.24 \mathrm{E}+01$ & 4.73E-01 & $0.00 \mathrm{E}+00$ & $2.78 \mathrm{E}+02$ & $7.59 E+01$ & $7.93 E+01$ \\
\hline GAMMA ONLY RAD/HR & $1.75 E+01$ & $3.71 \mathrm{E}-01$ & & $1.60 E+02$ & $5.72 E+01$ & $6.03 \mathrm{E}+01$ \\
\hline FOR THE LAYERED ORGANIC CASE: & & & & & & \\
\hline SUPERNATE Ba-137 CURIES & $7.54 \mathrm{E}+03$ & $1.20 \mathrm{E}+03$ & $0.00 \mathrm{E}+00$ & $1.81 E+05$ & $1.38 E+05$ & $1.68 \mathrm{E}+05$ \\
\hline SUPERNATE EU-154 CURIES & $1.38 \mathrm{E}+00$ & $1.18 \mathrm{E}-06$ & $0.00 \mathrm{E}+00$ & $0.00 \mathrm{E}+00$ & $0.00 E+00$ & $0.00 E+00$ \\
\hline RAD/HR TO ORGANIC LAYER & & & & & 27.7 & 32.6 \\
\hline
\end{tabular}


ENERGY DEPOSITION IN DST

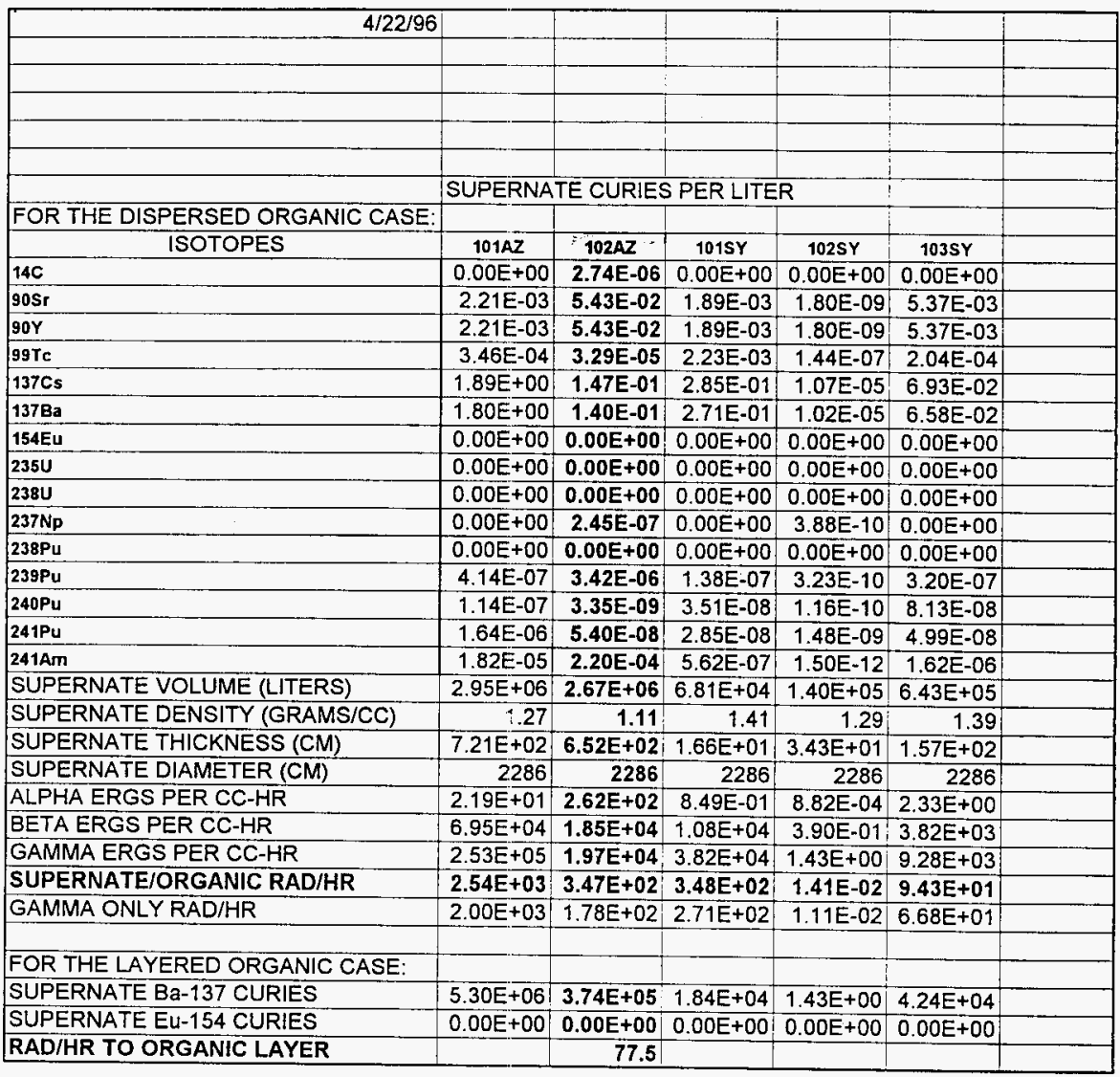




\begin{tabular}{|c|c|c|c|c|c|}
\hline $4 / 22 / 96$ & TANK & SUPERNATE & DENSITY & Cs-137 & Sr-90 \\
\hline & $103 \mathrm{C}$ & $503,000 \mathrm{~L}$ & \begin{tabular}{|r|}
1.07 \\
\end{tabular} & $.064 \mathrm{Ci} / \mathrm{L}$ & $2.5 \mathrm{E}-4 \mathrm{Ci} / \mathrm{L}$ \\
\hline & $103 \mathrm{U}$ & $6.28 \mathrm{E}+5 \mathrm{~L}$ & 1.385 . & $.376 \mathrm{Ci} / \mathrm{L}$ & $.0105 \mathrm{Ci} / \mathrm{L}$ \\
\hline & $105 \mathrm{U}$ & $5.94 E+5 L$ & 1.35 . & $.295 \mathrm{Ci} / \mathrm{L}$ & $.0185 \mathrm{Ci} / \mathrm{L}$ \\
\hline & $109 \mathrm{U}$ & $6,06 \mathrm{E}+5 \mathrm{~L}$ & 1.365 . & $.3885 \mathrm{Ci} / \mathrm{L}$ & $.0003 \mathrm{Ci} / \mathrm{L}$ \\
\hline & \multirow{2}{*}{\multicolumn{5}{|c|}{ ASSUME NO OTHER RADIONUCLIDES FOR THE ABOVE }} \\
\hline FOR THE DISPERSED ORGANIC CASE: & & & & & \\
\hline ISOTOPES & & & & & \\
\hline $14 \mathrm{C}$ & \multicolumn{5}{|c|}{ THICKNESS OF SUPERNATE $=$ VOLUME $(\mathrm{L}) / 4094.5(\mathrm{LCM})^{-}$} \\
\hline gost & & & & & \\
\hline $90 Y$ & & & & & \\
\hline 99Te & & & & & \\
\hline $137 \mathrm{Cs}$ & & & & & \\
\hline $137 \mathrm{Ba}$ & & & & & \\
\hline 154Eu & & & & & \\
\hline $235 \mathrm{U}$ & & & & & \\
\hline 238U & & & & & \\
\hline $237 \mathrm{~Np}$ & & & & & \\
\hline 238Pu & & & & & \\
\hline 239Pu & & & & & \\
\hline 240Pu & & & & & \\
\hline 241Pu & & & & & \\
\hline 241Am & & & & & \\
\hline SUPERNATE VOLUME (LITERS) & & & & & \\
\hline SUPERNATE DENSITY (GRAMS/CC) & & & & & \\
\hline SUPERNATE THICKNESS (CM) & & & & & \\
\hline SUPERNATE DIAMETER (CM) & & & & & \\
\hline ALPHA ERGS PER CC-HR & & & & & \\
\hline BETA ERGS PER CC-HR & & & & & \\
\hline GAMMA ERGS PER CC-HR & & & & & \\
\hline SUPERNATE/ORGANIC RAD/HR & & & & & \\
\hline GAMMA ONLY RAD/HR & & & & & \\
\hline FOR THE LAYERED ORGANIC CASE: & & & & & \\
\hline SUPERNATE $8 \mathrm{a}-137$ CURIES & & & & & \\
\hline SUPERNATE EU-154 CURIES & & & & & \\
\hline RAD/HR TO ORGANIC LAYER & & & & & \\
\hline
\end{tabular}


WHC-SD-WM-CN-050 Rev 0

Attachment 2

Sample Calculation for 105AP 


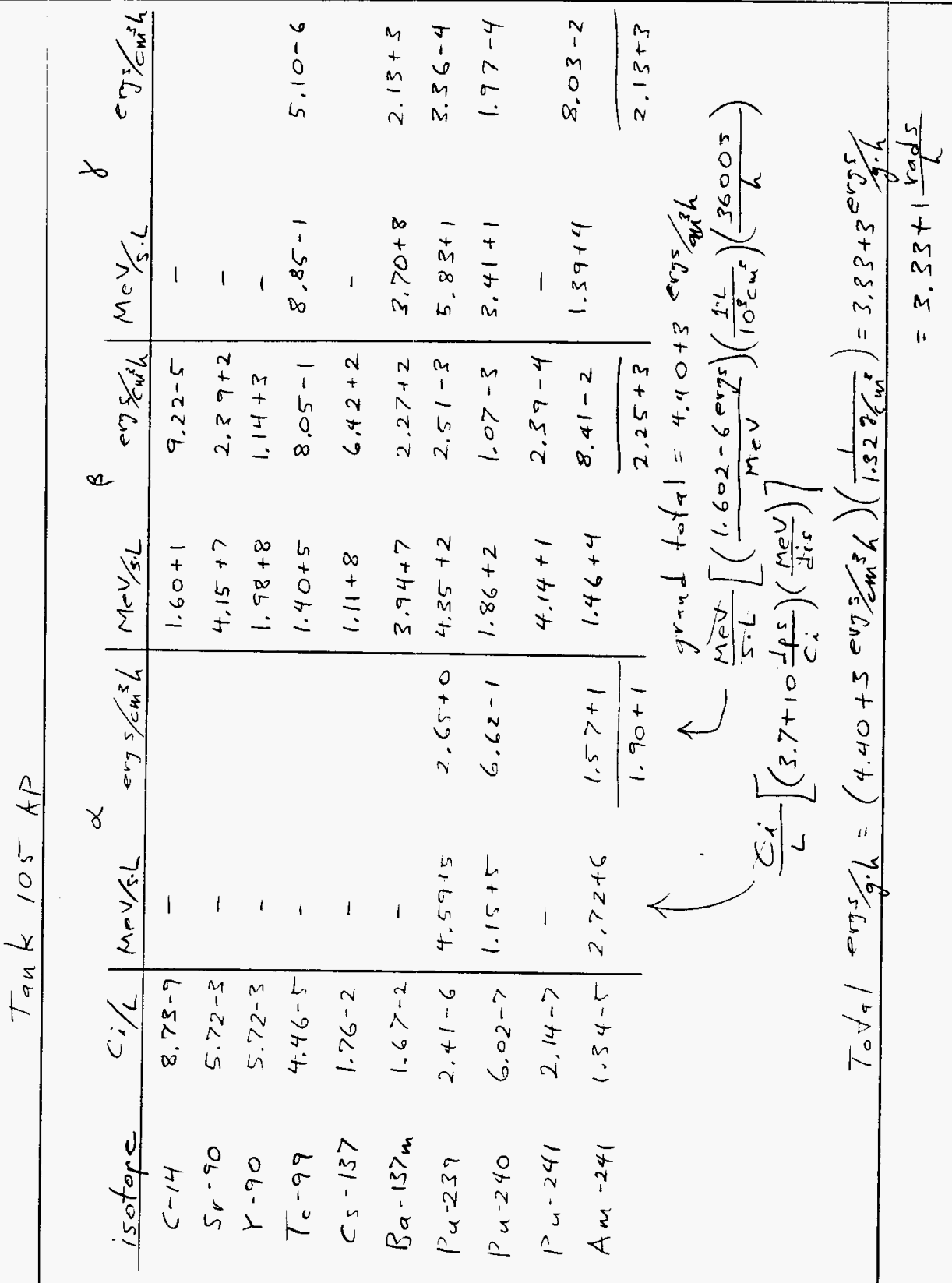




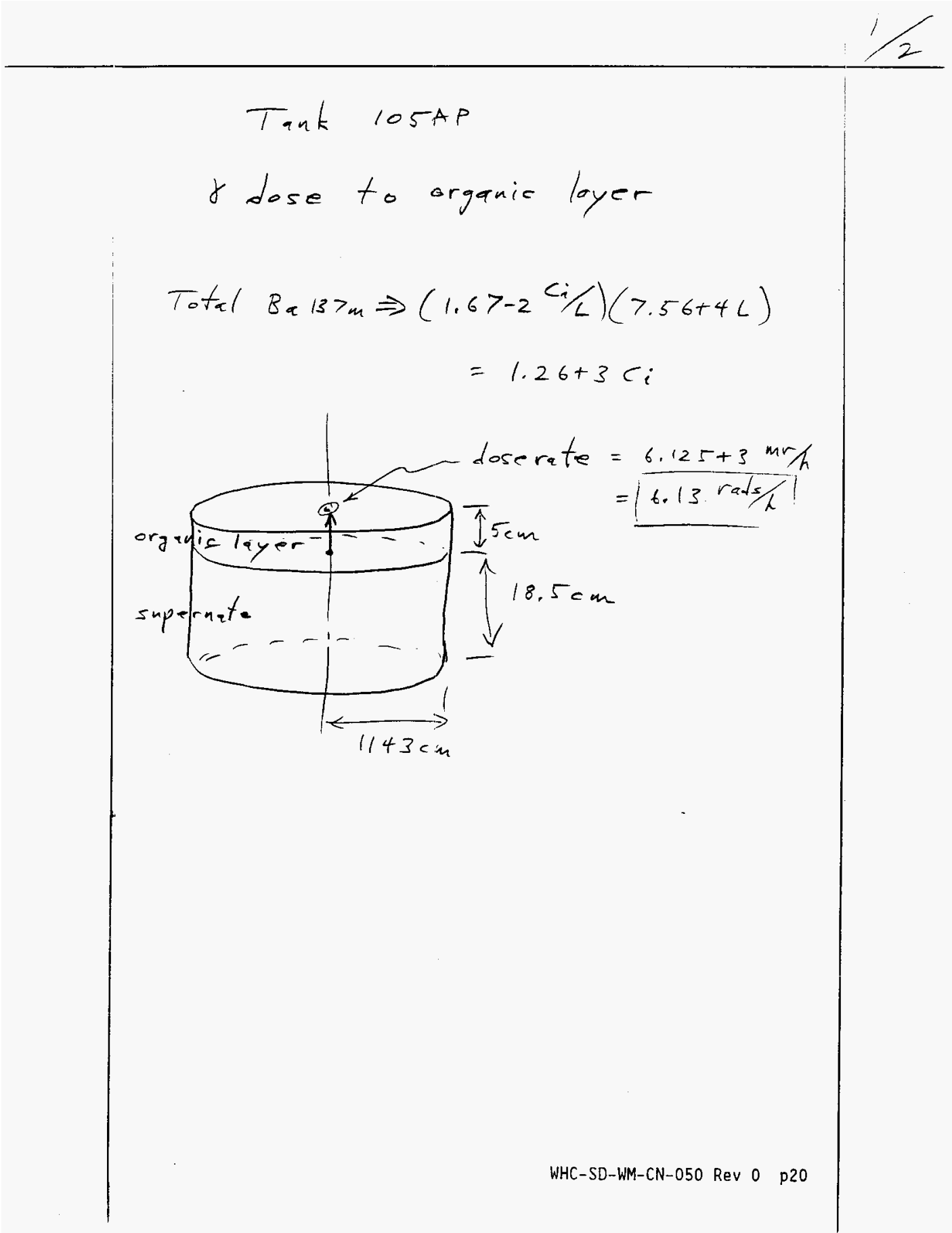


WHC-SD-WM-CN-050 Rev 0

\section{Attachment 3 \\ MICROSHIELD Files for Organic Layer Cases}


WHC-SD-WM-CN-050 Rev 0

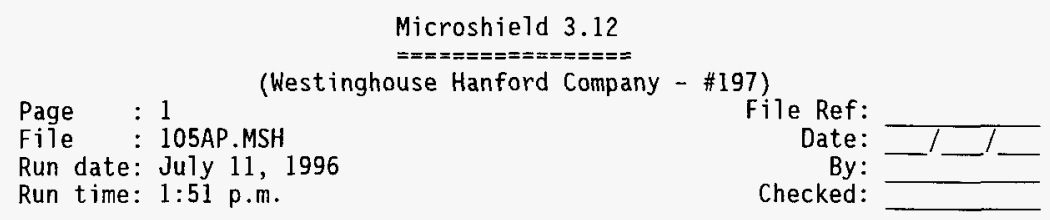

CASE: tanik 105AP organic dose

GEOMETRY 10: Cylindrical source from end - slab shields

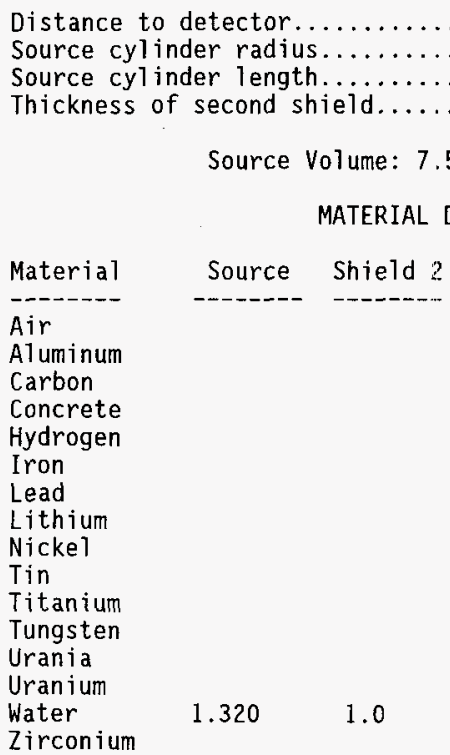




$$
\text { WHC-SD-WM-CN-050 Rev } 0
$$

Page 2

File: 105AP.MSH

CASE: tank 105AP organic dose

BUILDUP FACTOR: based on TAYLOR method.

Using the characteristics of the materials in shield 1.

\section{INTEGRATION PARAMETERS:}

Number of angle segments (Npsi)........... 15

Number of radial segments (Nradius)......... 15

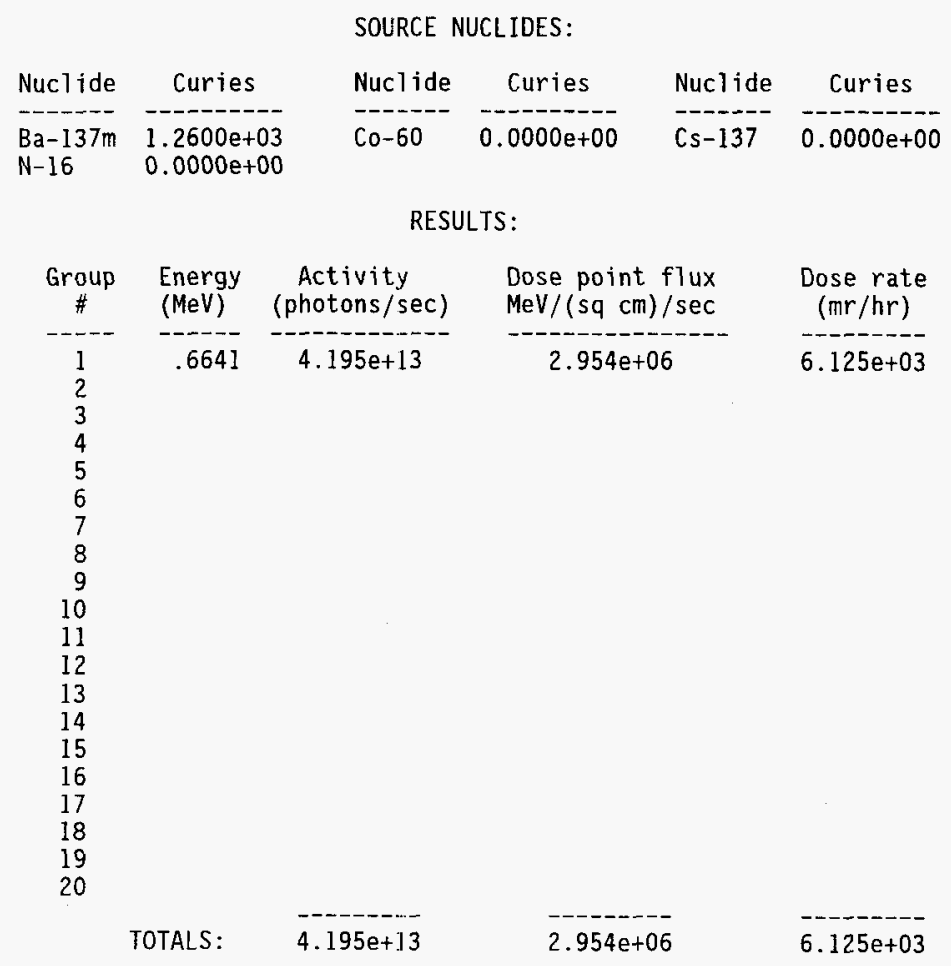




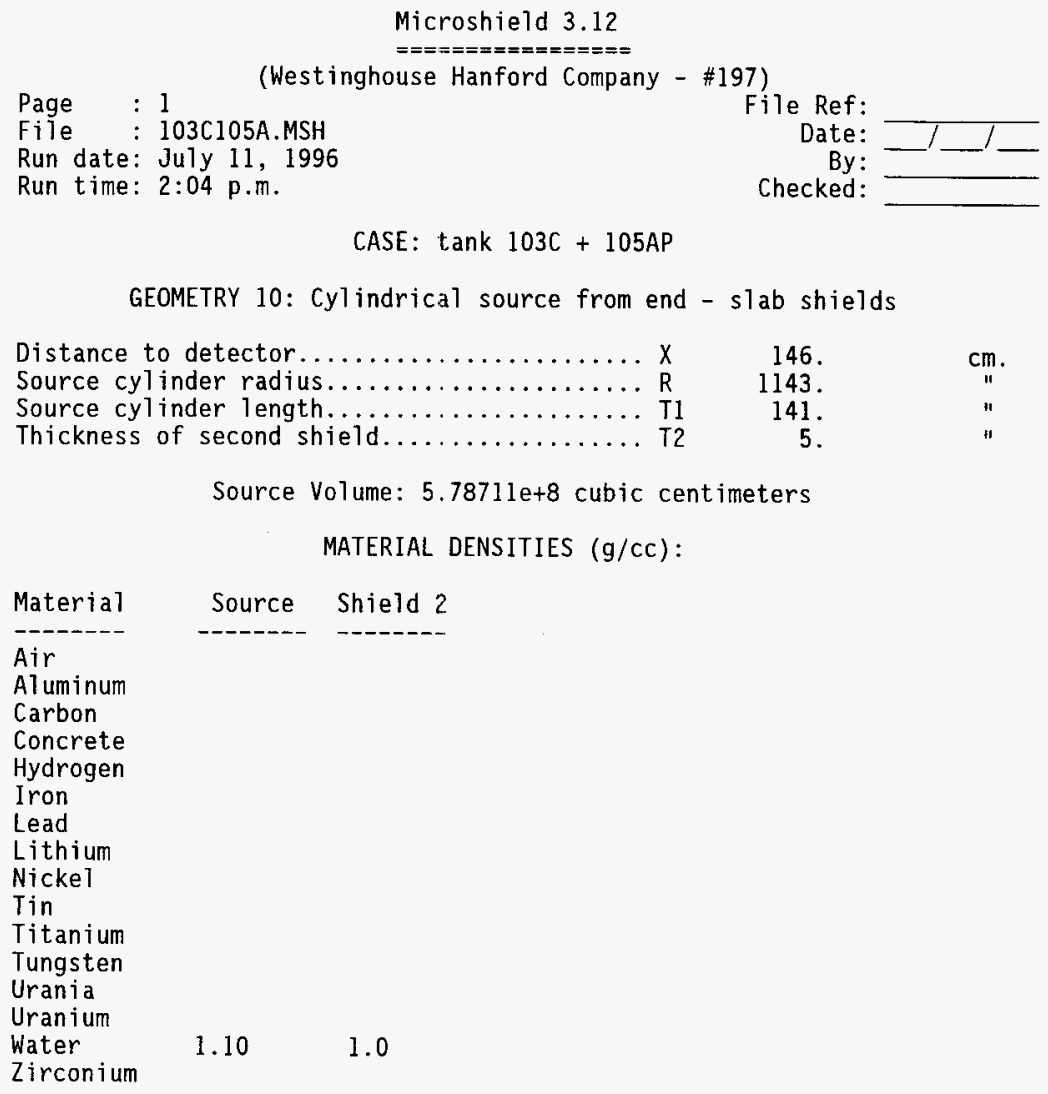




\begin{abstract}
BUILDUP FACTOR: based on TAYLOR method. Using the characteristics of the materials in shield 1 .
\end{abstract}

INTEGRATION PARAMETERS:

Number of angle segments (Npsi)........... 15

Number of radial segments (Nradius).......... 15

\begin{tabular}{|c|c|c|c|c|c|}
\hline \multirow[b]{2}{*}{ Nucl ide } & \multirow[b]{2}{*}{ Curies } & \multicolumn{2}{|c|}{ SOURCE NUCLIDES: } & \multirow[b]{2}{*}{ Nucl ide } & \multirow[b]{2}{*}{ Curies } \\
\hline & & Nuclide & Curies & & \\
\hline $\begin{array}{l}\text { Ba-137m } \\
N-16\end{array}$ & $\begin{array}{l}3.1900 \mathrm{e}+04 \\
0.0000 \mathrm{e}+00\end{array}$ & $\mathrm{Co}-60$ & $0.0000 e+00$ & Cs-137 & $0.0000 e+00$ \\
\hline
\end{tabular}

RESULTS:

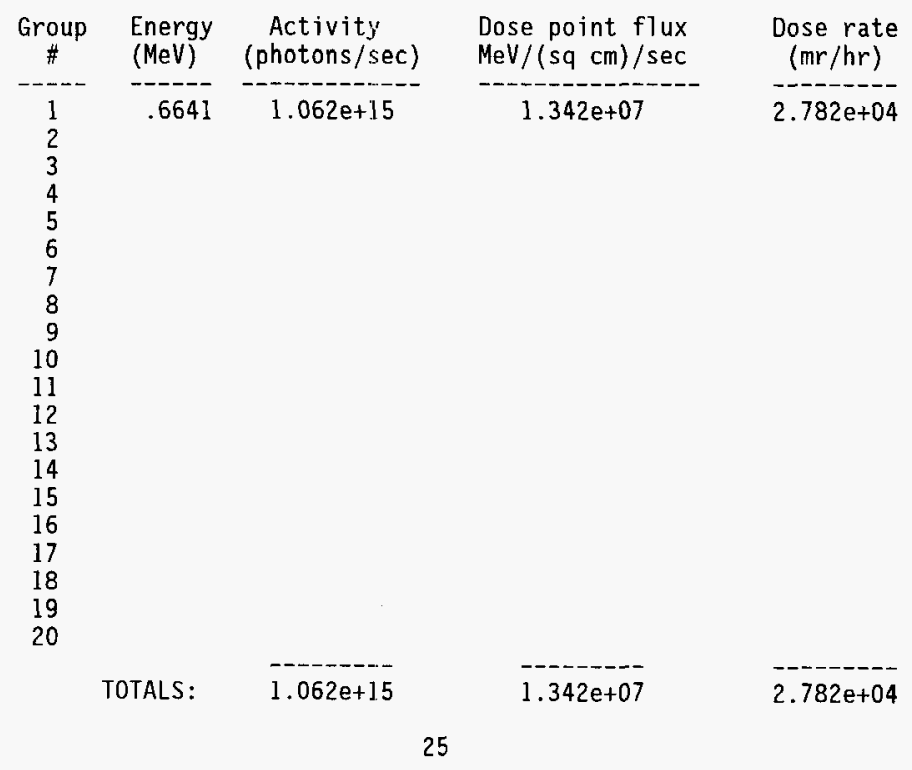




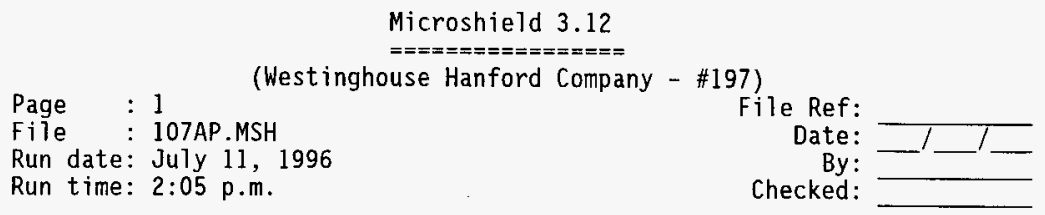

CASE: tank 107AP

GEOMETRY 10: Cylindrical source from end - slab shields

Distance to detector................... X

Source cylinder radius..................... $R$

Source cylinder length................... II

Thickness of second shield............... T2

33.6

1143.

28.6

5.
$\mathrm{cm}$.
II
"1

Source Volume: $1.17384 \mathrm{e}+8$ cubic centimeters

MATERIAL DENSITIES $(\mathrm{g} / \mathrm{cc})$ :

\begin{tabular}{lll} 
Material & Source & Shield ? \\
\hline Air & & \\
Aluminum & & \\
Carbon & & \\
Concrete & & \\
Hydrogen & & \\
Iron & & \\
Lead & & \\
Lithium & & \\
Nicke1 & & \\
Tin & \\
Titanium & & \\
Tungsten & & \\
Urania & & \\
Uranium & & \\
Water & 1.010 & 1.0 \\
Zirconium & &
\end{tabular}


WHC-SD-WM-CN-050 Rev 0

Page 2

CASE: tank 107AP

File: 107AP.MSH

BUILDUP FACTOR: based on TAYLOR method.

Using the characteristics of the materials in shield 1.

INTEGRATION PARAMETERS:

Number of angle segments (Npsi)............ 15

Number of radial segments (Nradius) .......... 15

SOURCE NUCLIDES:

\begin{tabular}{|c|c|c|c|c|c|}
\hline Nucl ide & Curies & Nucl ide & Curies & Nucl ide & Curies \\
\hline $\begin{array}{l}3 a-137 \\
u-154\end{array}$ & $\begin{array}{r}9.7700 \mathrm{e}-04 \\
3.8400 \mathrm{e}-04\end{array}$ & $\begin{array}{l}\mathrm{C} 0-60 \\
\mathrm{~N}-16\end{array}$ & $\begin{array}{l}0.0000 \mathrm{e}+00 \\
0.0000 \mathrm{e}+00\end{array}$ & Cs & \\
\hline
\end{tabular}

RESULTS:

\begin{tabular}{|c|c|c|c|c|}
\hline$\underset{\#}{\text { Group }}$ & $\begin{array}{l}\text { Energy } \\
(\mathrm{MeV})\end{array}$ & $\begin{array}{c}\text { Activity } \\
\text { (photons/sec) }\end{array}$ & $\begin{array}{l}\text { Dose point flux } \\
\mathrm{MeV} /(\mathrm{sq} \mathrm{cm}) / \mathrm{sec}\end{array}$ & $\begin{array}{c}\text { Dose rate } \\
(\mathrm{mr} / \mathrm{hr})\end{array}$ \\
\hline $\begin{array}{r}1 \\
2 \\
3 \\
4 \\
5 \\
6 \\
7 \\
8 \\
9 \\
10 \\
11 \\
12 \\
13 \\
14 \\
15 \\
16 \\
17 \\
18 \\
19 \\
20\end{array}$ & $\begin{array}{r}1.2993 \\
1.0051 \\
.8417 \\
.6676 \\
.5189 \\
.4453 \\
.3984 \\
.2422 \\
.1953 \\
.1172\end{array}$ & $\begin{array}{l}5.690 \mathrm{e}+0.6 \\
4.108 \mathrm{e}+06 \\
2.609 \mathrm{e}+06 \\
3.669 \mathrm{e}+07 \\
6.707 \mathrm{e}+04 \\
7.161 \mathrm{e}+04 \\
2.975 \mathrm{e}+04 \\
9.380 \mathrm{e}+05 \\
3.228 \mathrm{e}+04 \\
5.749 \mathrm{e}+06\end{array}$ & $\begin{array}{l}6.163 \mathrm{e}-01 \\
3.355 \mathrm{e}-01 \\
1.847 \mathrm{e}-01 \\
2.287 \mathrm{e}+00 \\
3.746 \mathrm{e}-03 \\
3.412 \mathrm{e}-03 \\
1.198 \mathrm{e}-03 \\
1.725 \mathrm{e}-02 \\
4.308 \mathrm{e}-04 \\
3.470 \mathrm{e}-02\end{array}$ & $\begin{array}{l}1.119 \mathrm{e}-03 \\
6.469 \mathrm{e}-04 \\
3.682 \mathrm{e}-04 \\
4.738 \mathrm{e}-03 \\
7.680 \mathrm{e}-06 \\
6.978 \mathrm{e}-06 \\
2.458 \mathrm{e}-06 \\
3.349 \mathrm{e}-05 \\
7.896 \mathrm{e}-07 \\
5.477 \mathrm{e}-05\end{array}$ \\
\hline & OTALS: & $5.598 \mathrm{e}+07$ & $3.484 \mathrm{e}+00$ & $6.979 e-03$ \\
\hline
\end{tabular}


WHC-SD-WM-CN-050 Rev 0

Microshield 3.12

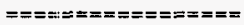

(Westinghouse Hanford Company - \#197)

Page : 1
File $: 103$ C107A.MSH
Run date: July 11, 1996
Run time: $2: 06 \mathrm{p.m}$.

File Ref:

Date:

By:

Checked:

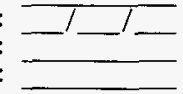

CASE: tank $103 C+107 A P$

GEOMETRY 10: Cylindrical source from end - slab shields

Distance to detector.................. 156 .

Source cylinder radius.................... $R \quad 1143$.

Source cylinder length................... TI 151.

Thickness of second shield............... T2 5 .

cm.

II

II

"

Source Volume: $6.19754 \mathrm{e}+8$ cubic centimeters

MATERIAL DENSITIES $(g / c c)$ :

\begin{tabular}{lll} 
Material & Source & Shield 2 \\
\hline Air & & \\
Aluminum & & \\
Carbon & & \\
Concrete & & \\
Hydrogen & & \\
Iron & & \\
Lead & & \\
Lithium & & \\
Nicke1 & & \\
Tin & & \\
Titanium & & \\
Tungsten & & \\
Urania & & \\
Uranium & & \\
Water & 1.060 & 1.0 \\
Zirconium & &
\end{tabular}




$$
\text { WHC-SD-WM-CN-050 Rev } 0
$$

Page 2

$$
\text { CASE: tank } 103 \mathrm{C}+107 \mathrm{AP}
$$

File: 103C107A.MSH

BUILDUP FACTOR: based on TAYLOR method.

Using the characteristics of the materials in shield 1 .

INTEGRATION PARAMETERS:

Number of angle segments (Npsi)............ 15

Number of radial segments (Nradius) .......... 15

SOURCE NUCLIDES:

\begin{tabular}{llllll} 
Nuclide & Curies & Nuciide & Curies & Nuclide & Curies \\
\hdashline $\mathrm{Ba}-137 \mathrm{~m}$ & $\mathbf{3 . 0 6 0 0 \mathrm { e } + 0 4}$ & $-\mathrm{C} 0-60$ & $0.0000 \mathrm{e}+00$ & $\mathrm{CS}-137$ & $0.0000 \mathrm{e}+00$ \\
$\mathrm{~N}-16$ & $0.0000 \mathrm{e}+00$ & & & &
\end{tabular}

RESULTS:

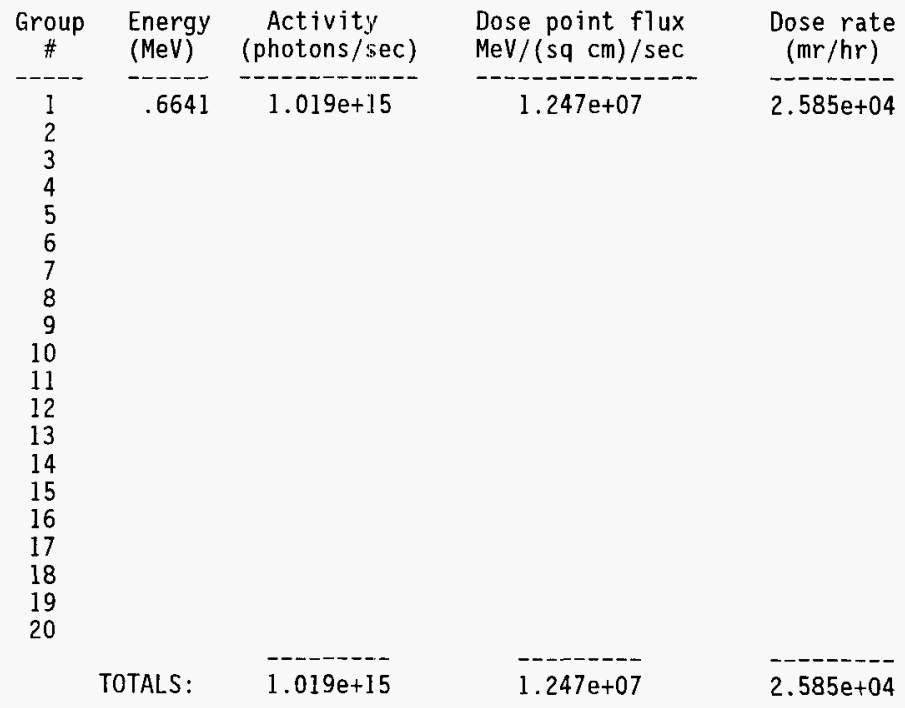


WHC-SD-WM-CN-050 Rev 0

Microshield 3.12

$==x=x=x=========$

(Westinghouse Hanford Company - \#197)

Page : 1

File : 103U105U.MSH

Run date: July 11, 1996

Run time: $2: 24$ p.m.

File Ref:

Date:

By:

Checked:

CASE : $\operatorname{tank} 103 \mathrm{U}+105 \mathrm{U}+109 \mathrm{U}+107 \mathrm{AP}$

GEOMETRY 10: Cylindrical source from end - slab shields

Distance to detector................. X 480 .

Source cylinder radius................... R 1143 .

Source cylinder length.................. TI 475.

Thickness of second shield................ T2

CIII.

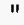

If

II

Source Volume: $1.94956 \mathrm{e}+9$ cubic centimeters

MATERIAL DENSITIES $(g / \mathrm{cc})$ :

\begin{tabular}{lll} 
Material & Source & Shield 2 \\
\hline Air & & \\
Aluminum & \\
Carbon & \\
Concrete & \\
Hydrogen & \\
Iron & \\
Lead & \\
Lithium & \\
Nicke] & \\
Tin & \\
Titanium & \\
Tungsten & \\
Urania & & \\
Uranium & \\
Water & 1.350 & \\
Zirconium & & \\
&
\end{tabular}


Page 2

CASE: $\operatorname{tank} 103 U+105 U+109 U+107 A P$

File: 103U105U.MSH

BUILDUP FACTOR: based on TAYLOR method.

Using the characteristics of the materials in shield 1.

INTEGRATION PARAMETERS:

Number of angle segments (Npsi) ............ 15

Number of radial segments (Nradius) .......... $\quad 15$

\begin{tabular}{llllll}
\multicolumn{7}{c}{ SOURCE NUCLIDES: } \\
Nuclide & Curies & Nuclide & \multicolumn{1}{c}{ Curies } & Nuclide & Curies \\
\hline Ba-137m & $6.1400 \mathrm{e}+05$ & $-0-60$ & $0.0000 \mathrm{e}+00$ & CS-137 & $0.0000 \mathrm{e}+00$ \\
$\mathrm{~N}-16$ & $0.0000 \mathrm{e}+00$ & & & &
\end{tabular}

RESULTS:

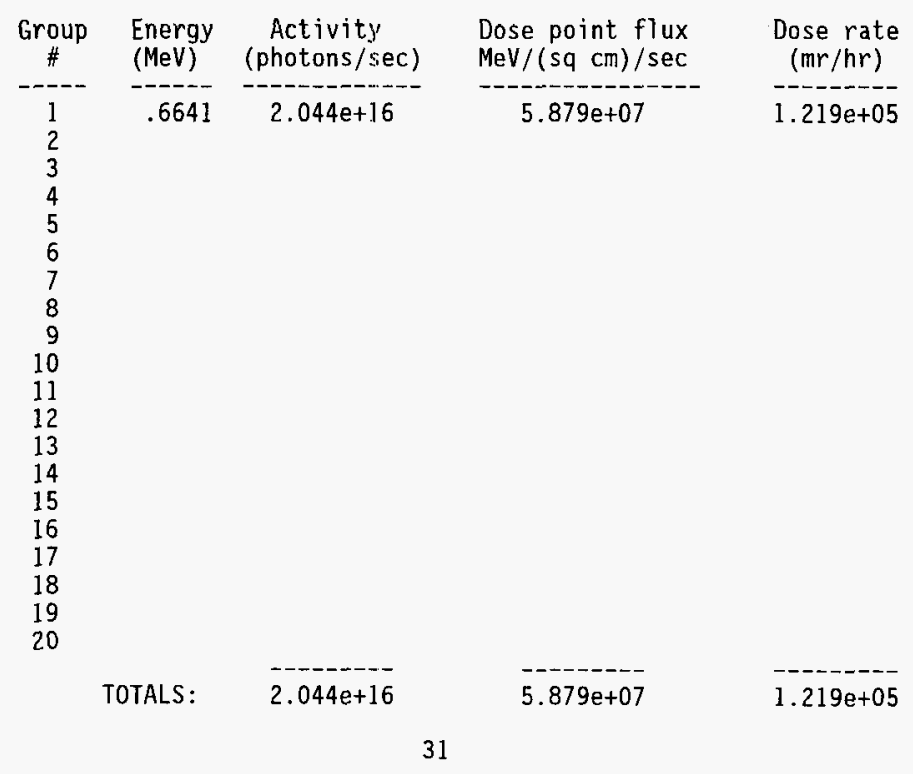


WHC-SD-WM-CN-050 Rev 0

Microshield 3.12

$====== \pm====== \pm==$

(Westinghouse Hanford Company - \#197)

Page : 1

File : 103C103U.MSH

Run date: July 11, 1996

Run time: 2:08 p.m.
File Ref:

Date:

By:

Checked:

CASE : $\operatorname{tank} 103 \mathrm{C}+103 \mathrm{U}+105 \mathrm{U}+109 \mathrm{U}+107 \mathrm{AP}$

GEOMETRY 10: Cylindrical source from end - slab shields

Distance to detector................. 603 .

Source cylinder radius................... R 1143 .

Source cylinder length................. T1 598.

Thickness of second shield.............. T2 5 .

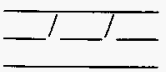

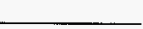

Source Volume: $2.45439 \mathrm{e}+9$ cubic centimeters

MATERIAL DENSITIES $(\mathrm{g} / \mathrm{cc})$ :

\begin{tabular}{lll} 
Material & Source & Shield 2 \\
\hline Air & & \\
Aluminum & & \\
Carbon & & \\
Concrete & \\
Hydrogen & \\
Iron & \\
Lead & \\
Lithium & \\
Nickel & \\
Tin & \\
Titanium & & \\
Tungsten & & \\
Urania & & \\
Uranium & & \\
Water & 1.290 & 1.0 \\
Zirconium & &
\end{tabular}


Page 2

File: 103C103U.MSH

CASE: $\operatorname{tank} 103 C+103 U+105 U+109 U+107 A P$

BUILDUP FACTOR: based on TAYLOR method.

Using the characteristics of the materials in shield 1 .

\section{INTEGRATION PARAMETERS:}

Number of angle segments (Npsi) ........... 15

Number of radial segments (Nradius) ......... 15

\section{SOURCE NUCLIDES:}

\begin{tabular}{lccccc} 
Nuclide & Curies & Nuclide & Curies & Nuclide & Curies \\
\hdashline $\begin{array}{ll}\text { Ba-137m } \\
N-16\end{array}$ & $6.4500 e+05$ & Co-60 & $0.0000 \mathrm{e}+00$ & $\mathrm{Cs}-137$ & $0.0000 \mathrm{e}+00$
\end{tabular}

RESULTS:

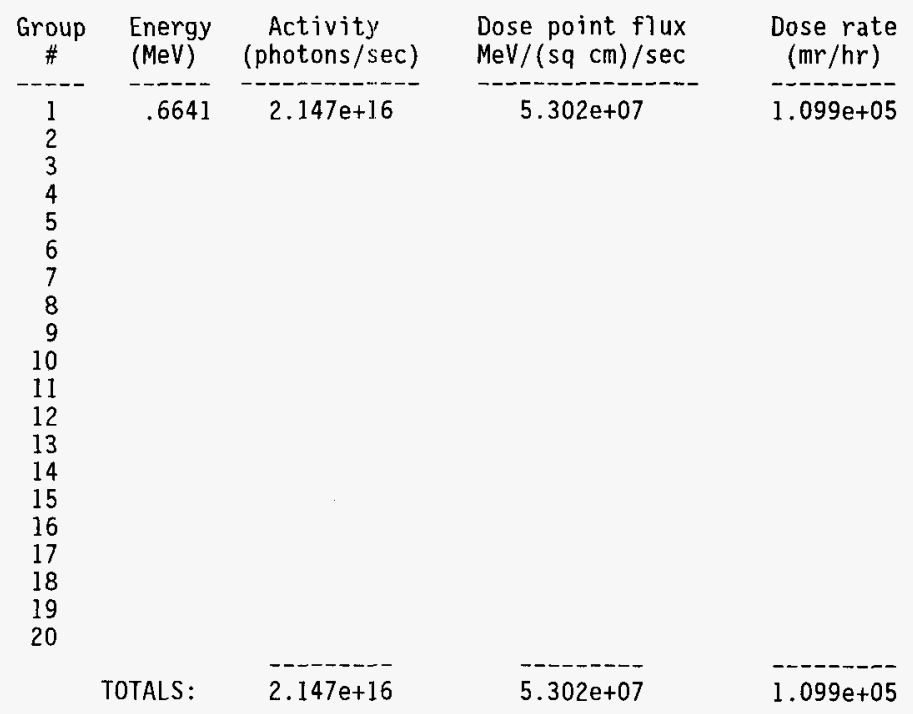


WHC-SD-WM-CN-050 Rev 0

Microshield 3.12

$===:==== \pm====== \pm$

(Westinghouse Hanford Company - \#197)

Page : 1

File : 102AY.MSH

Run date: July 11, 1996

Run time: 2:10 p.m.

File Ref:

Date:

By:

Checked:

CASE: $\operatorname{tank}$ 102AY

GEOMETRY 10: Cylindrical source from end - slab shields

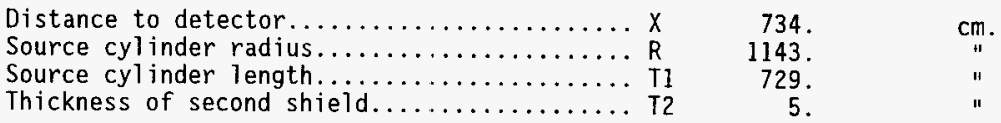

Source Volume: $2.99206 \mathrm{e}+9$ cubic centimeters

MATERIAL DENSITIES $(\mathrm{g} / \mathrm{cc})$ :

Material Source Shield 2

Air

Aluminum

Carbon

Concrete

Hydrogen

Iron

Lead

Lithium

Nickel

Tin

Titanium

Tungsten

Urania

Uranium

$\begin{array}{lll}\text { Water } & 1.140 & 1.0\end{array}$

Zirconium 
Page 2

CASE: tank 102AY

File: 102AY.MSH

BUILDUP FACTOR: based on TAYLOR method.

Using the characteristics of the materials in shield 1 .

INTEGRATION PARAMETERS:

Number of angle segments (Npsi) ........... 15

Number of radial segments (Nradius).......... 15

SOURCE NUCLIDES:

\begin{tabular}{lccccc} 
Nuclide & Curies & Nuclide & Curies & Nuclide & Curies \\
\hdashline $\mathrm{Ba}-137 \mathrm{~m}$ & $1.3800 \mathrm{e}+05$ & $\mathrm{C}-60$ & $0.0000 \mathrm{e}+00$ & $\mathrm{Cs}-137$ & $0.0000 \mathrm{e}+00$ \\
$\mathrm{~N}-16$ & $0.0000 \mathrm{e}+00$ & & & &
\end{tabular}

RESULTS :

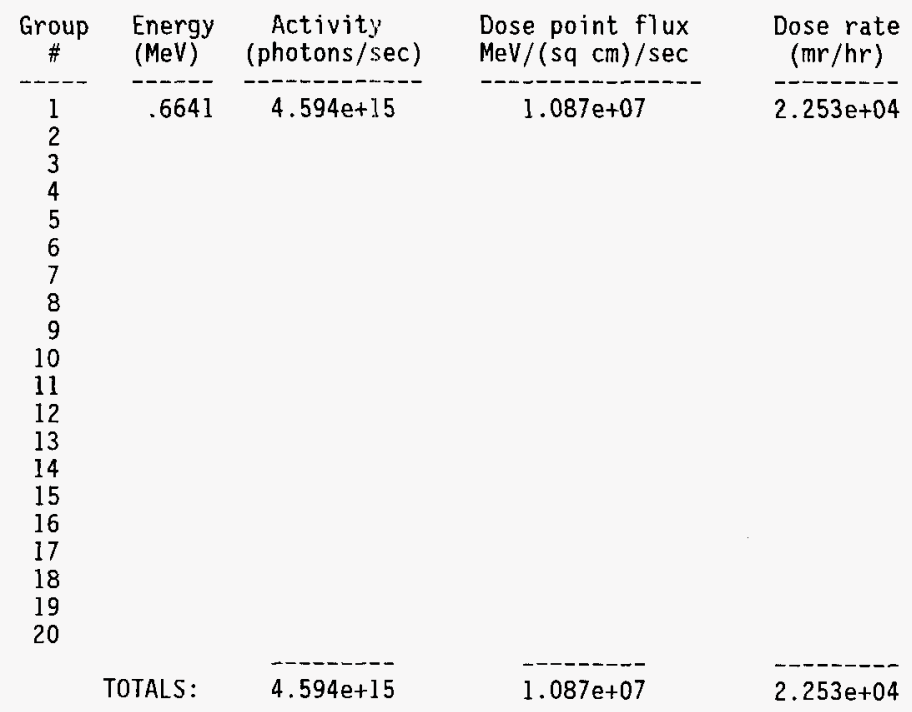


Microshield 3.12

$================$

\begin{tabular}{llr}
\multicolumn{3}{c}{ (Westinghouse Hanford Company - \#197) } \\
Page $: 1103$ File Ref: \\
File $: 102 \mathrm{~A}$. MSH & Date: \\
Run date: July 11,1996 & By: \\
Run time: & $2: 30 \mathrm{p} . \mathrm{m}$. & Checked:
\end{tabular}

CASE: $\operatorname{tank} 103 \mathrm{C}+102 \mathrm{AY}$

GEOMETRY 10: Cylindrical source from end - slab shields

Distance to detector................... 855 .

Source cylinder radius...................... $R \quad 1143$.

Source cylinder length.................... T1 850.

Thickness of second shield................ T2 "

Source Volume: $3.48868 \mathrm{e}+9$ cubic centimeters

MATERIAL DENSITIES $(\mathrm{g} / \mathrm{cc})$ :

\begin{tabular}{lll} 
Material & Source & Shield 2 \\
\hline Air & & \\
Aluminum & & \\
Carbon & & \\
Concrete & & \\
Hydrogen & & \\
Iron & \\
Lead & & \\
Lithium & \\
Nickel & & \\
Tin & & \\
Titanium & & \\
Tungsten & & \\
Urania & & \\
Uranium & & \\
Water & 1.130 & 1.0 \\
Zirconium & &
\end{tabular} 
Page 2

CASE: tank $103 C+102 A Y$

File: 103C102A.MSH

BUILDUP FACTOR: based on TAYLOR method.

Using the characteristics of the materials in shield 1 .

INTEGRATION PARAMETERS:

Number of angle segments (Npsi)........... 15

Number of radial segments (Nradius)......... 15

SOURCE NUCLIDES:

\begin{tabular}{llllll} 
Nuclide & Curies & Nuc 7 ide & Curies & Nuclide & Curies \\
\hdashline $\mathrm{Ba}-137 \mathrm{~m}$ & $1.6800 \mathrm{e}+05$ & $-\mathrm{C}-60$ & $0.0000 \mathrm{e}+00$ & $\mathrm{Cs}-137$ & $0.0000 \mathrm{e}+00$ \\
$\mathrm{~N}-16$ & $0.0000 \mathrm{e}+00$ & & & &
\end{tabular}

RESULTS:

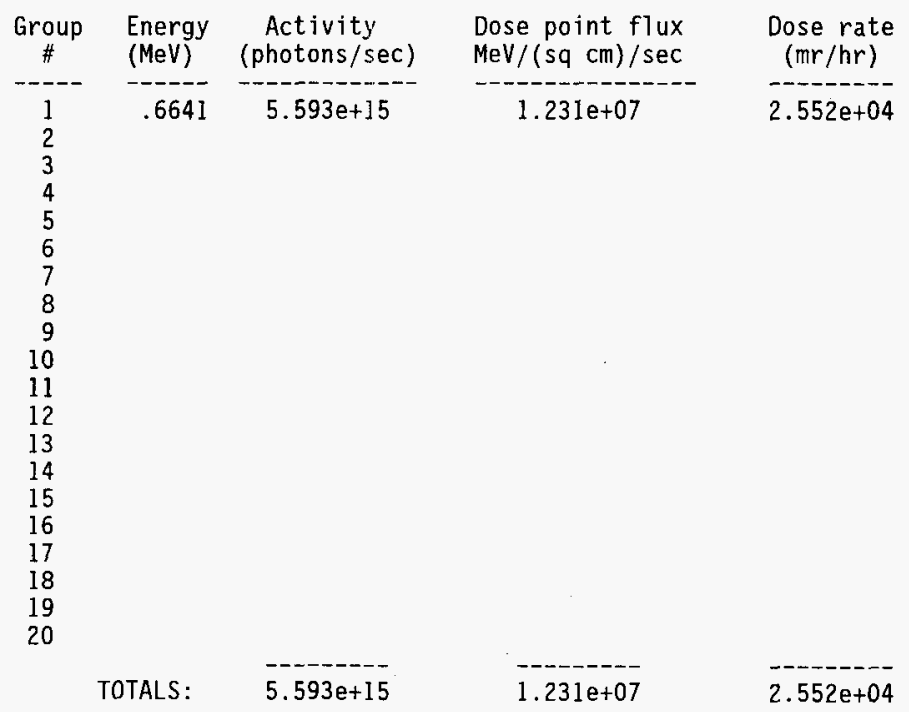


WHC-SD-WM-CN-050 Rev 0

Microshield 3.12

$==== \pm===========$

Page : 1

File : 102AZ.MSH

Run date: July 11, 1996

Run time: $2: 12$ p.m.

(Westinghouse Hanford Company - \#197)

File Ref:

Date:

By:

Checked:

CASE: $\operatorname{tank} 102 A Z$

GEOMETRY 10: Cylindrical source from end - slab shields

Distance to detector.................. X 657 .

Source cylinder radius..................... R 1143.

Source cylinder length................... T1 652 .

Thickness of second shield............... T2 5 .

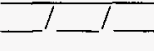

Source volume: $2.67602 \mathrm{e}+9$ cubic centimeters

MATERIAL DENSITIES $(\mathrm{g} / \mathrm{cc})$ :

Material Source Shield $z$

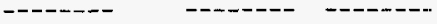

Air

Aluminum

Carbon

Concrete

Hydrogen

Iron

Lead

Lithium

Nickel

Tin

Titanium

Tungsten

Urania

Uranium

Water

$1.110 \quad 1.0$

Zirconium 
WHC-SD-WM-CN-050 Rev 0

Page 2

CASE: tank $102 A Z$

File: 102AZ.MSH

BUILDUP FACTOR: based on TAYLOR method.

Using the characteristics of the materials in shield $l$.

INTEGRATION PARAMETERS:

Number of angle segments (Npsi)........... 15

Number of radial segments (Nradius) ......... 15

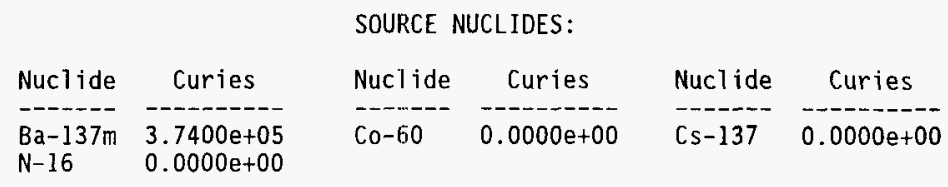

RESULTS:

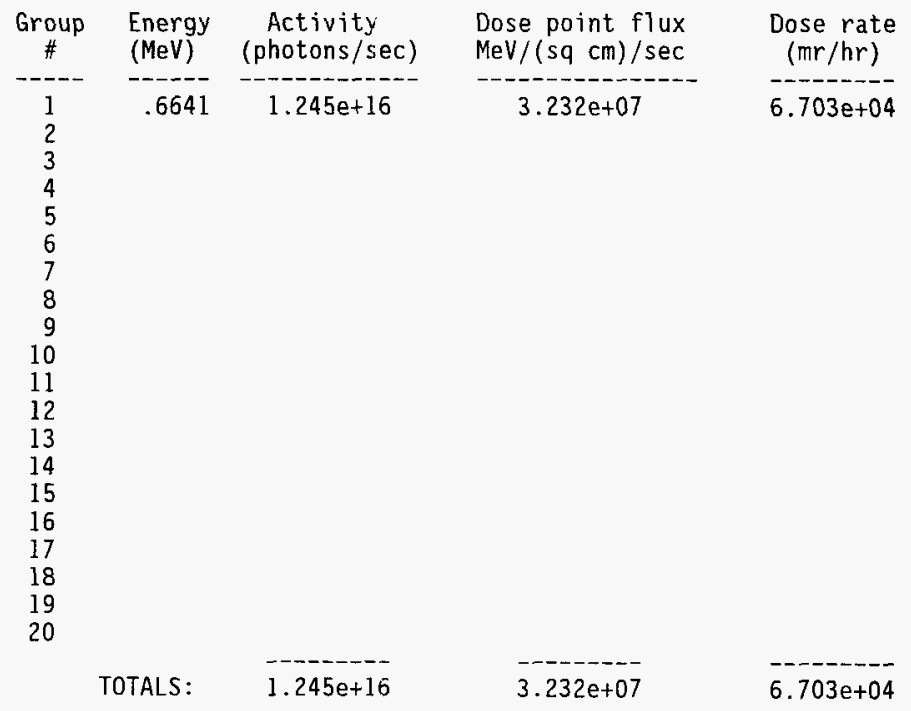


WHC-SD-WM-CN-050 Rev 0

Attachment 4

MICROSHIELD files for gamma leakage calculations 
Microshield 3.12

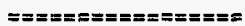

File: : 1 OCM.MSH

Run date: July 11, 1996

(Westinghouse Hanford Company - \#197)

Run time: 3:09 p.m.

File Ref:

Date:

By:

Checked:

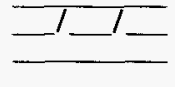

CASE : $0 \mathrm{~cm}$

GEOMETRY 14: Infinite slab source - slab shields

Distance to detector................ X

Source slab thickness................. Tl

Thickness of second shield............. T2

100.001

100.

0.001

cm.

"

II

MATERIAL DENSITIES $(g / c c)$ :

\begin{tabular}{lll} 
Material & Source & Shield 2 \\
\hline Air & & \\
A7uminum & \\
Carbon & \\
Concrete & \\
Hydrogen & \\
Iron & \\
Lead & \\
Lithium & \\
Nickel & \\
Tin & \\
Titanium & \\
Tungsten & \\
Urania & & \\
Uranium & \\
Water & 1.50 & \\
Zirconium & & \\
& &
\end{tabular}


BUILDUP FACTOR: based on TAYLOR method.

Using the characteristics of the materials in shield 1.

INTEGRATION PARAMETERS:

None - analytically integrated.

SOURCE NUCLIDES:

\begin{tabular}{|c|c|c|c|c|c|}
\hline Nuctide & Curies & Nucl ide & Curies & Nucl ide & Curies \\
\hline $\begin{array}{l}\mathrm{Ba}-137 \mathrm{~m} \\
\mathrm{~N}-16\end{array}$ & $\begin{array}{l}1.0000 \mathrm{e}-03 \\
0.0000 \mathrm{e}+00\end{array}$ & Co & 0.000 & 0 & .00 \\
\hline
\end{tabular}

\section{RESULTS:}

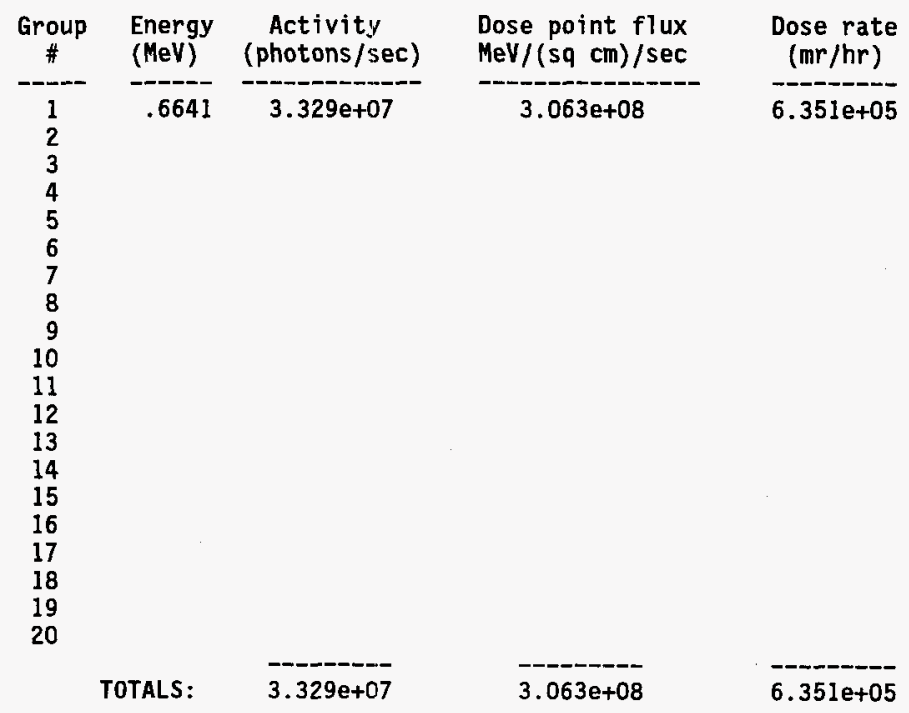


WHC-SD-WM-CN-050 Rev 0

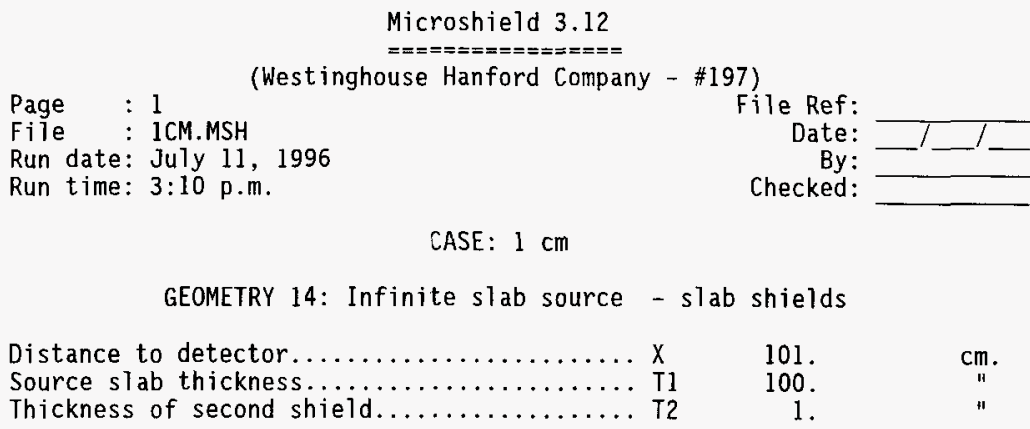

MATERIAL DENSITIES $(\mathrm{g} / \mathrm{cc})$ :

\begin{tabular}{lll} 
Material & Source & Shield 2 \\
\hline Air & & \\
Aluminum & & \\
Carbon & & \\
Concrete & & \\
Hydrogen & & \\
Iron & & \\
Lead & & \\
Lithium & & \\
Nicke1 & & \\
Tin & & \\
Titanium & & \\
Tungsten & & \\
Urania & & \\
Uranium & & \\
Water & 1.50 & 1.50 \\
Zirconium & &
\end{tabular}


WHC-SD-WM-CN-050 Rev 0

Page 2

CASE : $1 \mathrm{~cm}$

File: 1CM.MSH

BUILDUP FACTOR: based on TAYLOR method.

Using the characteristics of the materials in shield 1 .

INTEGRATION PARAMETERS:

None - analytically integrated.

SOURCE NUCLIDES:

\begin{tabular}{llllll} 
Nuclide & Curies & Nuclide & Curies & Nuclide & Curies \\
\hdashline $\mathrm{Ba}-137 \mathrm{~m}$ & $1.0000 \mathrm{e}-03$ & $\mathrm{C}-\mathrm{C}-60$ & $0.0000 \mathrm{e}+00$ & $\mathrm{Cs}-137$ & $0.0000 \mathrm{e}+00$ \\
$\mathrm{~N}-16$ & $0.0000 \mathrm{e}+00$ & & & &
\end{tabular}

RESULTS:

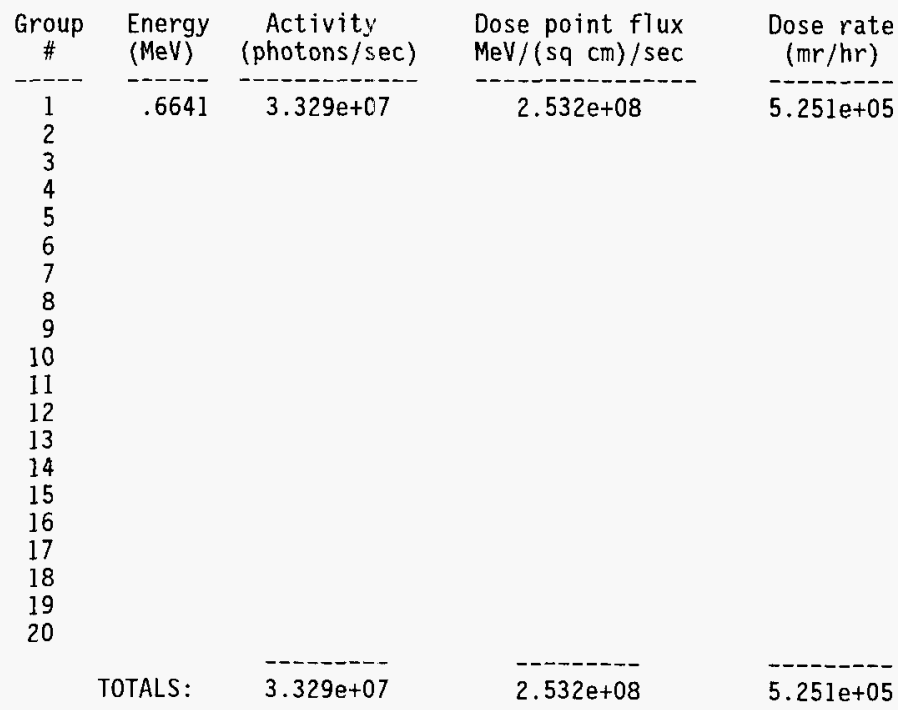


Microshield 3.12

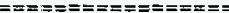

File : 1OCM.MSH

Run date: July 11, 1996

Run time: $3: 12$ p.m.

(Westinghouse Hanford Company - \#197)

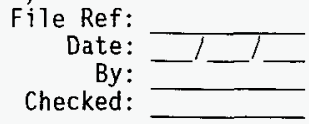

CASE : $10 \mathrm{~cm}$

GEOMETRY 14: Infinite slab source - slab shields

Distance to detector.................... 110.

Source slab thickness................... T1 100.

Thickness of second shield............... T2 10.

cm.

II

MATERIAL DENSITIES $(\mathrm{g} / \mathrm{cc})$ :

\begin{tabular}{lll} 
Material & Source & Shield ? \\
\hline Air & & \\
Aluminum & & \\
Carbon & & \\
Concrete & & \\
Hydrogen & & \\
Iron & & \\
Lead & & \\
Lithium & & \\
Nickel & & \\
Tin & & \\
Titanium & & \\
Tungsten & & \\
Urania & & \\
Uranium & & \\
Water & 1.50 & 1.50 \\
Zirconium & &
\end{tabular}


BUILDUP FACTOR: based on TAYLOR method. Using the characteristics of the materials in shield 1 .

INTEGRATION PARAMETERS:

None - analytically integrated.

SOURCE NUCLIDES:

\begin{tabular}{lccccc} 
Nuclide & Curies & Nuclide & Curies & Nuclide & Curjes \\
\hline Ba-137m & $1.0000 \mathrm{e}-03$ & Co-60 & $0.0000 \mathrm{e}+00$ & Cs-137 & $0.0000 \mathrm{e}+00$ \\
$\mathrm{~N}-16$ & $0.0000 \mathrm{e}+00$ & & & &
\end{tabular}

RESULTS:

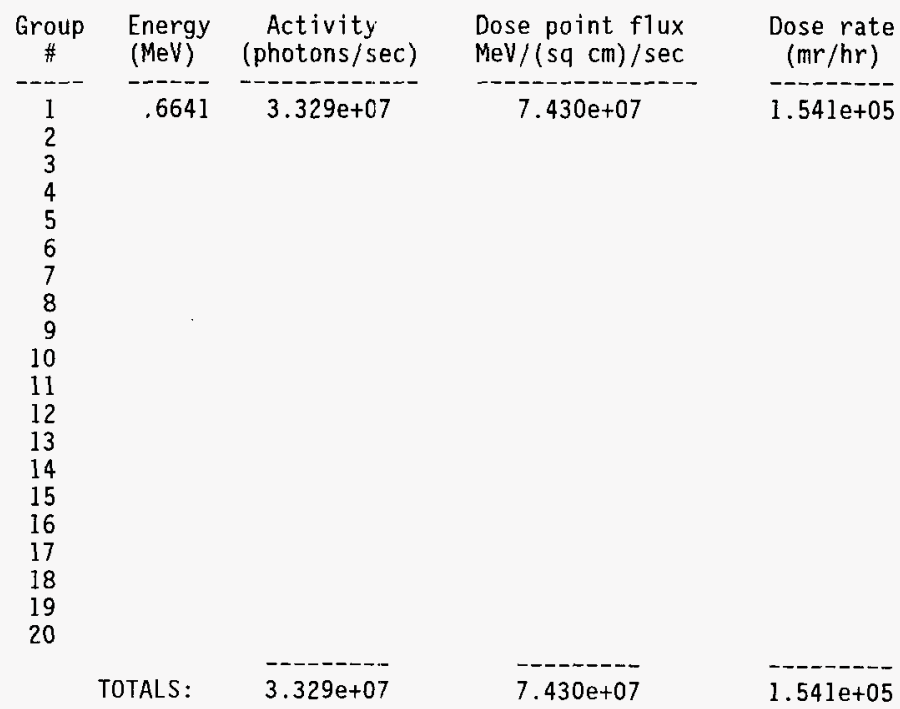


Microshield 3.12

$===x=== \pm===== \pm===$

File : 20CM.MSH

Run date: July 11, 1996

Run time: $3: 13$ p.m.

(Westinghouse Hanford Company - \#197)

File Ref:

Date:

By:

Checked:

CASE : $20 \mathrm{~cm}$

GEOMETRY 14: Infinite slab source - slab shields

Distance to detector.................. X

Source slab thickness................... T1

Thickness of second shield............... T2

120.

100.

20.

$\mathrm{cm}$.

$\$$

MATERIAL DENSITIES $(\mathrm{g} / \mathrm{cc})$ :

\begin{tabular}{lll} 
Material & Source & Shield 2 \\
\hline Air & & \\
Aluminum & & \\
Carbon & & \\
Concrete & & \\
Hydrogen & & \\
Iron & & \\
Lead & & \\
Lithium & & \\
Nickel & & \\
Tin & & \\
Titanium & & \\
Tungsten & & \\
Urania & & \\
Uranium & & \\
Water & 1.50 & 1.50 \\
Zirconium & &
\end{tabular}


Page 2

File: 20CM.MSH

CASE: $20 \mathrm{~cm}$

BUILDUP FACTOR: based on TAYLOR method. Using the characteristics of the materials in shield 1 .

INTEGRATION PARAMETERS:

None - analytically integrated.

SOURCE NUCLIDES:

\begin{tabular}{lclccc} 
Nuclide & Curies & Nuclide & Curies & Nuclide & Curies \\
\hdashline Ba-137m & $1.0000 \mathrm{e}-03$ & Co-60 & $0.0000 \mathrm{e}+00$ & Cs-137 & $0.0000 \mathrm{e}+00$ \\
$\mathrm{~N}-16$ & $0.0000 \mathrm{e}+00$ & & & &
\end{tabular}

RESULTS:

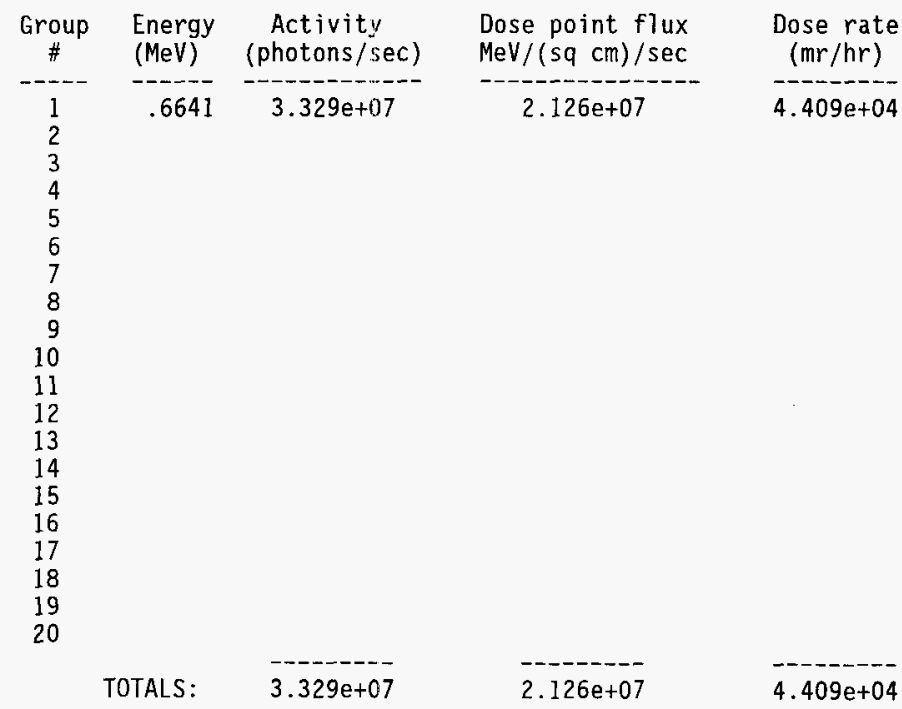


WHC-SD-WM-CN-050 Rev 0

Microshield 3.12

$==x============$

(Westinghouse Hanford Company - \#197)

Page : 1

File : 30CM.MSH

Run date: July 11, 1996

Run time: $3: 14 \mathrm{p} . \mathrm{m}$.

File Ref:

Date:

By:

Checked:

CASE: $30 \mathrm{~cm}$

GEOMETRY 14: Infinite slab source - slab shields

Distance to detector................... 130 .

Source slab thickness................... Tl 100.

Thickness of second shield............... T2 30 .

$\mathrm{cm}$.

MATERIAL DENSITIES $(\mathrm{g} / \mathrm{cc})$ :

Material Source Shield 2

Air

Aluminum

Carbon

Concrete

Hydrogen

Iron

Lead

Lithium

Nicke1

Tin

Titanium

Tungsten

Urania

Uranium

Water

$1.50 \quad 1.50$

Zirconium 
WHC-SD-WM-CN-050 Rev 0

Page 2

$$
\text { CASE: } 30 \mathrm{~cm}
$$

File: 30CM.MSH

BUILDUP FACTOR: based on TAYLOR method.

Using the characteristics of the materials in shield 1.

INTEGRATION PARAMETERS:

None - analytically integrated.

SOURCE NUCLIDES:

\begin{tabular}{lccccc} 
Nuclide & Curies & Nuclide & Curies & Nuclide & Curies \\
\hdashline $\mathrm{Ba}-137 \mathrm{~m}$ & $1.0000 \mathrm{e}-03$ & $\mathrm{C} 0-60$ & $0.0000 \mathrm{e}+00$ & $\mathrm{Cs}-137$ & $0.0000 \mathrm{e}+00$ \\
$\mathrm{~N}-16$ & $0.0000 \mathrm{e}+00$ & & & &
\end{tabular}

RESULTS:

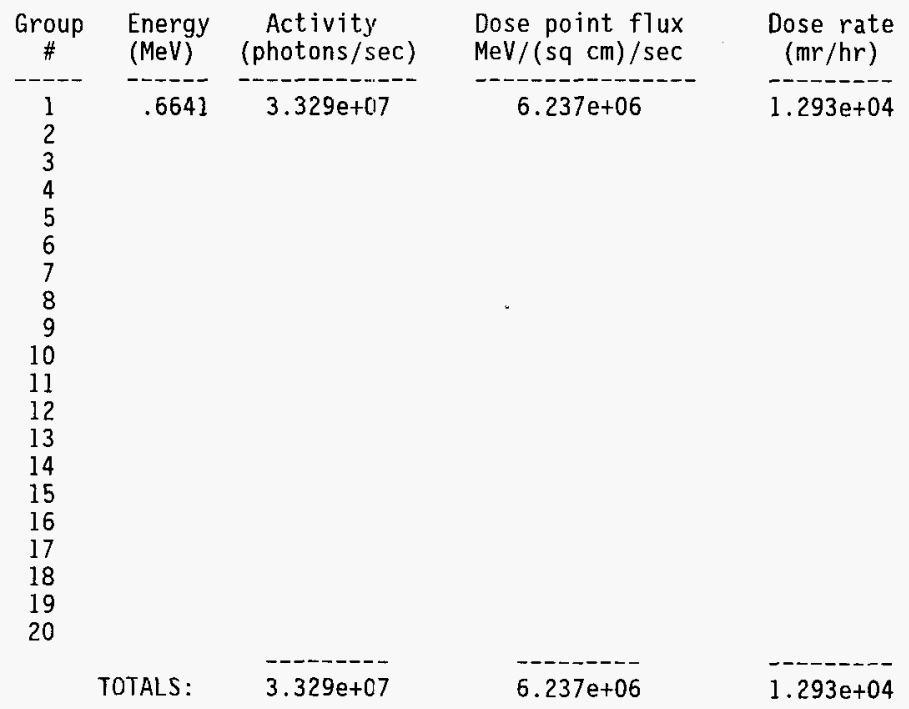


Microshield 3.12

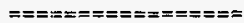

Page : 1

File : 40CM.MSH

Run date: Juty 11, 1996

(Westinghouse Hanford Company - \#197)

Run time: $3: 15$ p.m.

File Ref:

Date:

By:

Checked:

CASE : $40 \mathrm{~cm}$

GEOMETRY 14: Infinite slab source - slab shields

Distance to detector...................

140.

Source slab thickness.................. Tl

100.

Thickness of second shield...

T2

40 .

$\mathrm{cm}$.

II

II

MATERIAL DENSITIES $(g / c c)$ :

\begin{tabular}{lll} 
Material & Source & Shield 2 \\
\hline Air & & \\
Aluminum & & \\
Carbon & \\
Concrete & \\
Hydrogen & \\
Iron & \\
Lead & \\
Lithium & \\
Nickel & \\
Tin & \\
Titanium & \\
Tungsten & & \\
Urania & & \\
Uranium & & \\
Water & 1.50 & \\
Zirconium & &
\end{tabular}


WHC-SD-WM-CN-050 Rev 0

Page 2

FiTe: 40CM.MSH

CASE : $40 \mathrm{~cm}$

BUILDUP FACTOR: based on TAYLOR method.

Using the characteristics of the materials in shield 1.

\section{INTEGRATION PARAMETERS:}

None - analytically integrated.

\section{SOURCE NUCLIDES:}

\begin{tabular}{llllll} 
Nuclide & Curies & Nuclide & Curies & Nuclide & Curies \\
\hdashline $\mathrm{Ba}-137 \mathrm{~m}$ & $1.0000 \mathrm{e}-03$ & $\mathrm{C}-6-60$ & $0.0000 \mathrm{e}+00$ & $\mathrm{Cs}-137$ & $0.0000 \mathrm{e}+00$ \\
$\mathrm{~N}-16$ & $0.0000 \mathrm{e}+00$ & & & &
\end{tabular}

RESULTS:

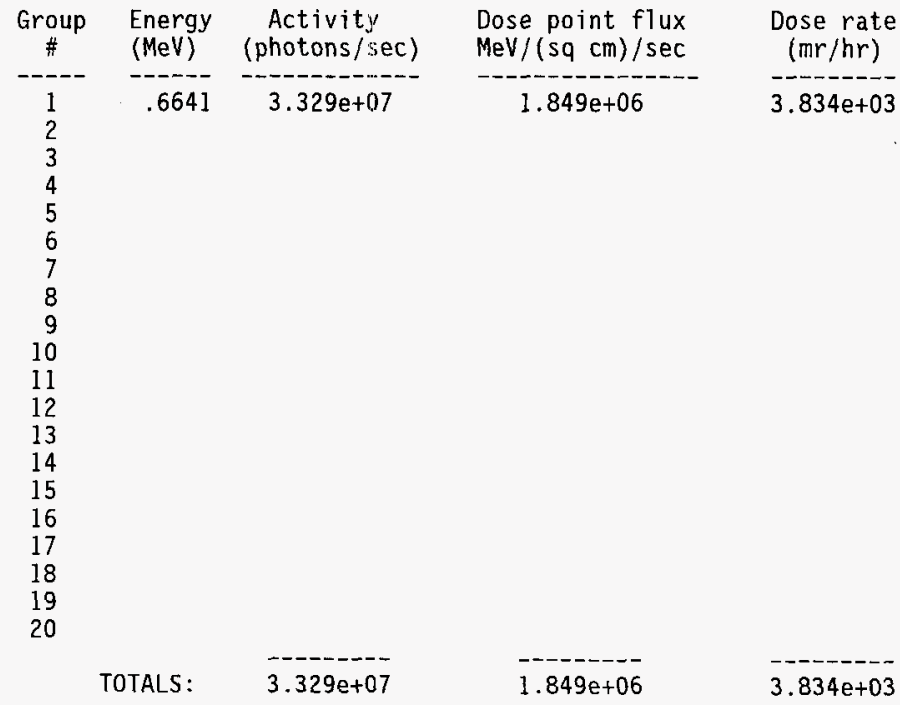


WHC-SD-WM-CN-050 Rev 0

Microshield 3.12

$====== \pm== \pm== \pm====$

File : 50CM.MSH

Run date: July 11, 1996

Run time: $3: 15$ p.m.

(Westinghouse Hanford Company - \#197)

File Ref:

Date:

By:

Checked:

CASE : $50 \mathrm{~cm}$

GEOMETRY 14: Infinite slab source - slab shields

Distance to detector................... X

Source slab thickness................... Tl

Thickness of second shield................ T2

150.

100.

50 .

Cm.

II

MATERIAL DENSITIES $(\mathrm{g} / \mathrm{cc})$ :

\begin{tabular}{|c|c|c|}
\hline \multicolumn{3}{|l|}{ Material } \\
\hline \multicolumn{3}{|l|}{ Air } \\
\hline \multicolumn{3}{|l|}{ Aluminum } \\
\hline \multicolumn{3}{|l|}{ Carbon } \\
\hline \multicolumn{3}{|l|}{ Concrete } \\
\hline \multicolumn{3}{|l|}{ Hydrogen } \\
\hline \multicolumn{3}{|l|}{ Iron } \\
\hline \multirow{2}{*}{\multicolumn{3}{|c|}{$\begin{array}{l}\text { Lead } \\
\text { Lithium }\end{array}$}} \\
\hline & & \\
\hline \multicolumn{3}{|l|}{ Nickel } \\
\hline \multirow{2}{*}{\multicolumn{3}{|c|}{ Tin }} \\
\hline & & \\
\hline \multicolumn{3}{|l|}{ Tungsten } \\
\hline \multicolumn{3}{|l|}{ Urania } \\
\hline Uranium & & \\
\hline Water & 1.50 & 1.50 \\
\hline
\end{tabular}


Page 2

File: 50CM.MSH

\section{CASE : $50 \mathrm{~cm}$}

BUILDUP FACTOR: based on TAYLOR method.

Using the characteristics of the materials in shield 1 .

INTEGRATION PARAMETERS:

None - analytically integrated.

SOURCE NUCLIDES:

\begin{tabular}{llllll} 
Nuclide & Curies & Nuclide & Curies & Nuclide & Curies \\
\hline $\mathrm{Ba}-137 \mathrm{~m}$ & $1.0000 \mathrm{e}-03$ & $\mathrm{C}-60$ & $0.0000 \mathrm{e}+00$ & $\mathrm{Cs}-137$ & $0.0000 \mathrm{e}+00$ \\
$\mathrm{~N}-16$ & $0.0000 \mathrm{e}+00$ & & & &
\end{tabular}

RESULTS:

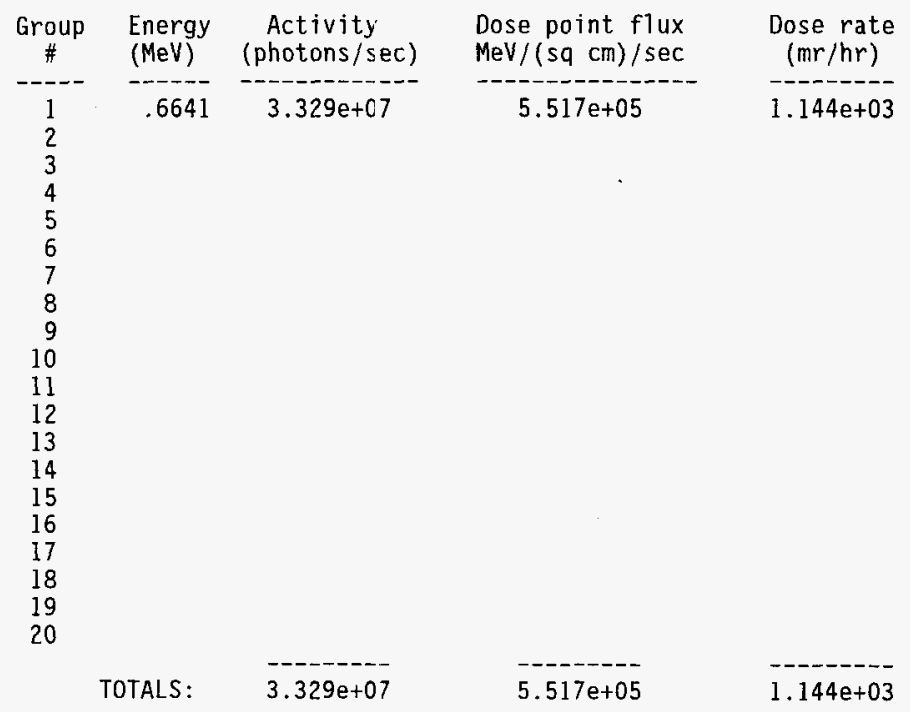




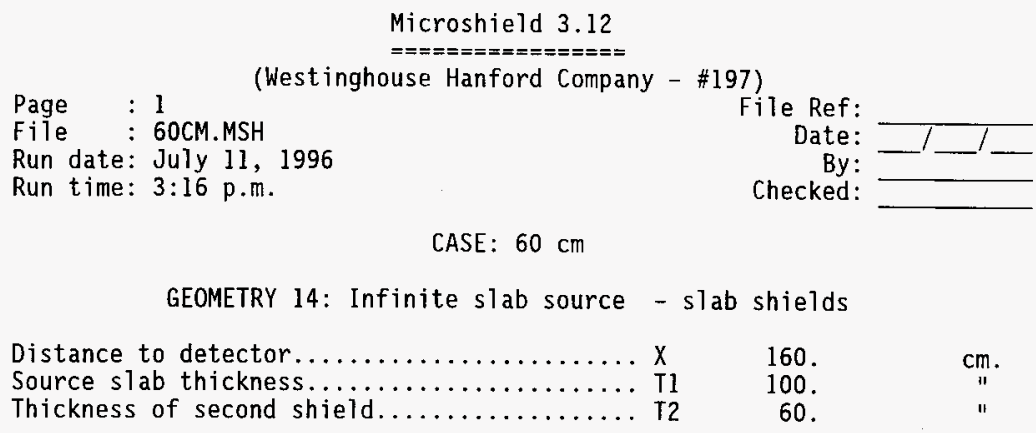

MATERIAL DENSITIES $(\mathrm{g} / \mathrm{cc})$ :

\begin{tabular}{lll} 
Material & Source & Shield 2 \\
\hline Air & & \\
Aluminum & & \\
Carbon & & \\
Concrete & & \\
Hydrogen & & \\
Iron & & \\
Lead & & \\
Lithium & & \\
Nickel & & \\
Tin & & \\
Titanium & & \\
Tungsten & & \\
Urania & & \\
Uranium & & \\
Water & 1.50 & 1.50 \\
Zirconium & &
\end{tabular}


Page 2

$$
\text { CASE : } 60 \mathrm{~cm}
$$

File: $60 \mathrm{cM} . \mathrm{MSH}$

BUILDUP FACTOR: based on TAYLOR method.

Using the characteristics of the materials in shield 1 .

INTEGRATION PARAMETERS:

None - analytically integrated.

SOURCE NUCLIDES:

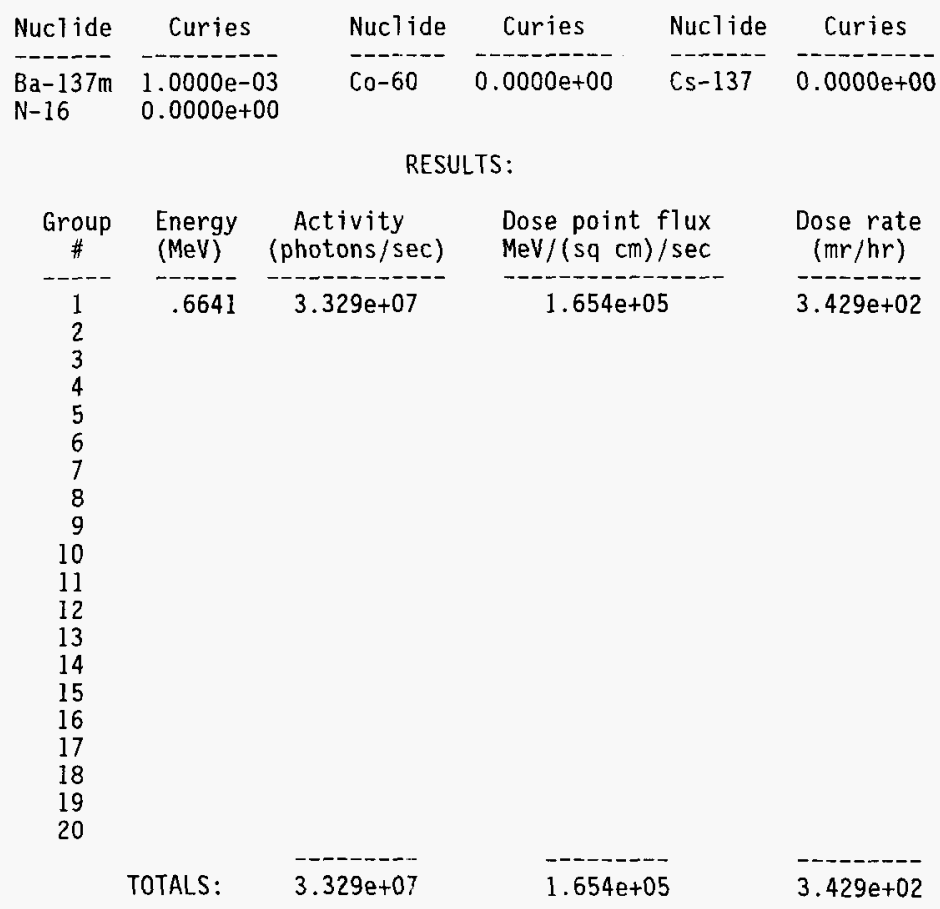


WHC-SD-WM-CN-050 Rev 0

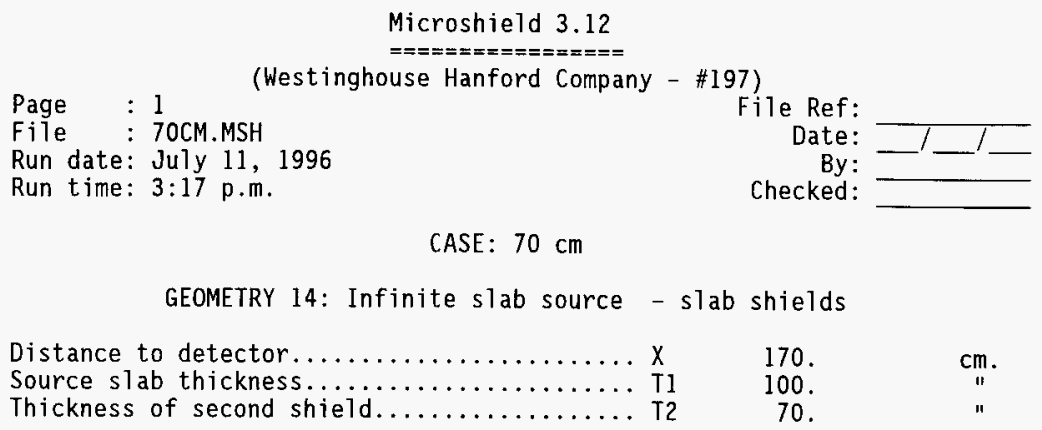

MATERIAL DENSITIES $(\mathrm{g} / \mathrm{cc})$ :

\begin{tabular}{lll} 
Material & Source & Shield 2 \\
\hline Air & & \\
Aluminum & & \\
Carbon & & \\
Concrete & & \\
Hydrogen & \\
Iron & & \\
Lead & & \\
Lithium & & \\
Nickel & & \\
Tin & & \\
Titanium & & \\
Tungsten & & \\
Urania & & \\
Uranium & & \\
Water & 1.50 & 1.50 \\
Zirconium & &
\end{tabular}


Page 2

$$
\text { CASE : } 70 \mathrm{~cm}
$$

BUILOUP FACTOR: based on TAYLOR method.

Using the characteristics of the materials in shield 1 .

\section{INTEGRATION PARAMETERS:}

None - analytically integrated.

SOURCE NUCLIDES:

\begin{tabular}{llllll} 
Nuclide & Curies & Nuclide & Curies & Nuclide & Curies \\
\hdashline Ba-137m & $1.0000 \mathrm{e}-03$ & C0-60 & $0.0000 \mathrm{e}+00$ & $\mathrm{Cs}-137$ & $0.0000 \mathrm{e}+00$ \\
$\mathrm{~N}-16$ & $0.0000 \mathrm{e}+00$ & & & &
\end{tabular}

RESULTS:

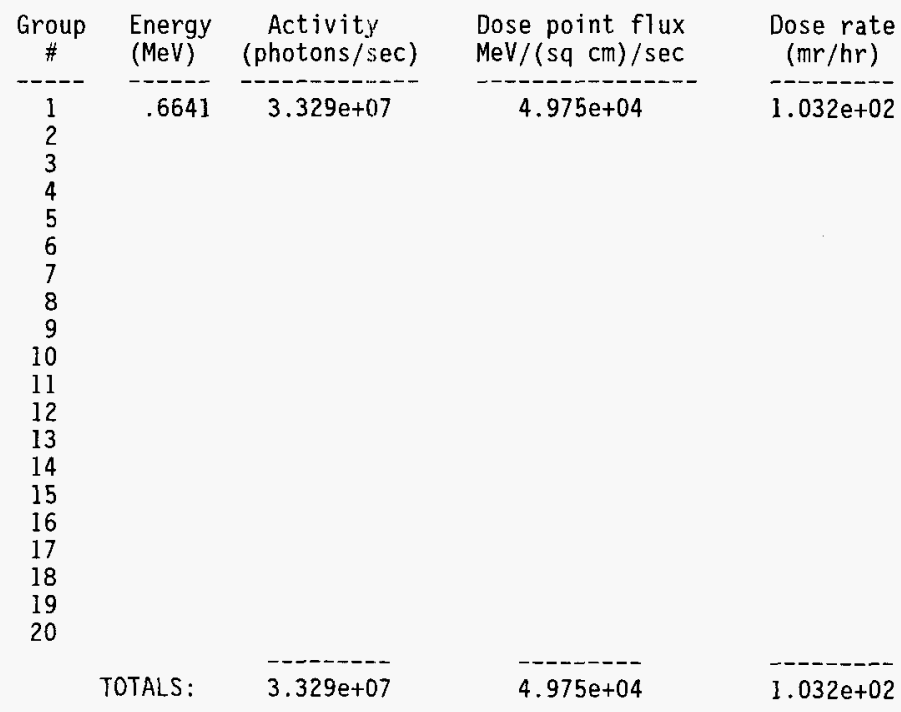


WHC-SD-WM-CN-050 Rev 0

Microshield 3.12

$x=m===========3 x$

(Westinghouse Hanford Company - \#197)
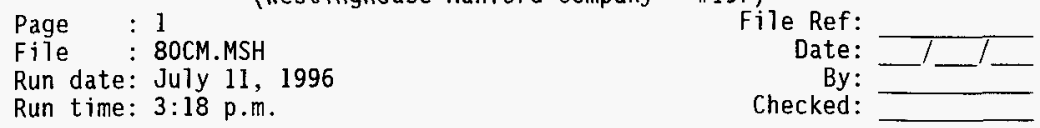

CASE : $80 \mathrm{~cm}$

GEOMETRY 14: Infinite slab source - slab shields

Distance to detector................... 180 .

Source slab thickness.................. T1 100.

Thickness of second shield............... T2 80 .

cm.

II

MATERIAL DENSITIES $(\mathrm{g} / \mathrm{cc})$ :

\begin{tabular}{lll} 
Material & Source & Shield 2 \\
\hline Air & & \\
Aluminum & & \\
Carbon & & \\
Concrete & & \\
Hydrogen & & \\
Iron & & \\
Lead & & \\
Lithium & & \\
Nicke1 & & \\
Tin & & \\
Titanium & & \\
Tungsten & & \\
Urania & & \\
Uranium & & \\
Water & 1.50 & 1.50 \\
Zirconium & &
\end{tabular}


Page 2

File: 80CM.MSH

CASE : $80 \mathrm{~cm}$

BUILDUP FACTOR: based on TAYLOR method.

Using the characteristics of the materials in shield 1 .

INTEGRATION PARAMETERS:

None - analytically integrated.

SOURCE NUCLIDES:

\begin{tabular}{lccccc} 
Nuclide & Curies & Nuclide & Curies & Nuclide & Curies \\
\hdashline Ba-137m & $1.0000 \mathrm{e}-03$ & Co-60 & $0.0000 \mathrm{e}+00$ & $\mathrm{Cs}-137$ & $0.0000 \mathrm{e}+00$ \\
$\mathrm{~N}-16$ & $0.0000 \mathrm{e}+00$ & & & &
\end{tabular}

RESULTS:

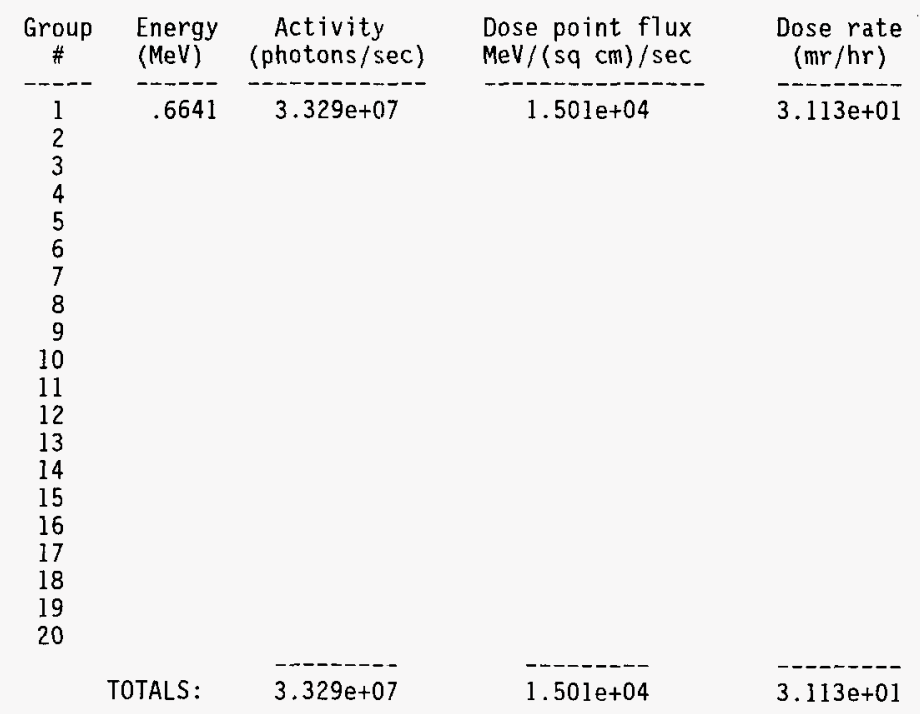




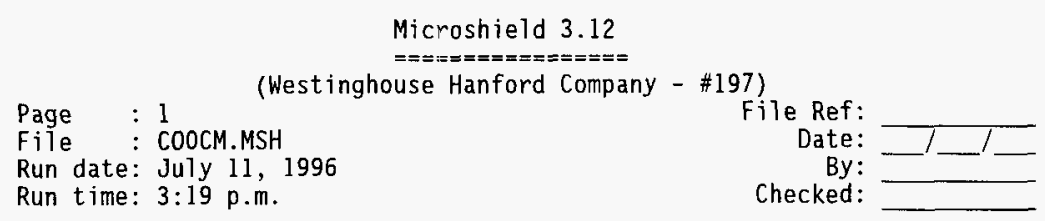

CASE: $0 \mathrm{~cm}$

GEOMETRY 14: Infinite slab source - slab shields

Distance to detector................... X

Source slab thickness................... T1

Thickness of second shield............... T2

100.001

100.

0.001

$\mathrm{cm}$.

MATERIAL DENSITIES $(g / c C)$ :

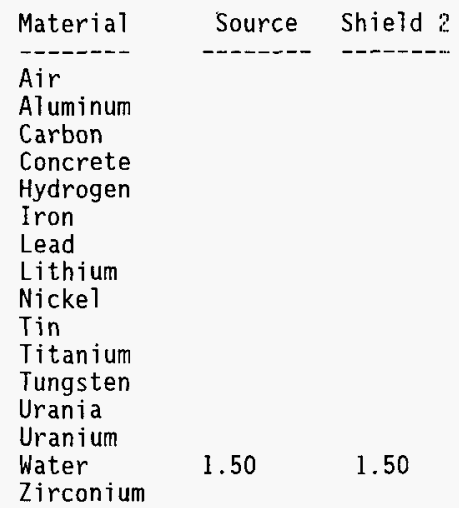


CASE : $0 \mathrm{~cm}$

BUILDUP FACTOR: based on TAYLOR method.

Using the characteristics of the materials in shield 1.

\section{INTEGRATION PARAMETERS:}

None - analytically integrated.

SOURCE NUCLIDES:

\begin{tabular}{llllll} 
Nuclide & \multicolumn{1}{c}{ Curies } & Nuclide & \multicolumn{1}{c}{ Curies } & Nuclide & Curies \\
\hline $\mathrm{Ba}-137 \mathrm{~m}$ & $0.0000 \mathrm{e}+00$ & $-0-60$ & $1.0000 \mathrm{e}-03$ & $\mathrm{Cs}-137$ & $0.0000 \mathrm{e}+00$ \\
$\mathrm{~N}-16$ & $0.0000 \mathrm{e}+00$ & & & &
\end{tabular}

RESULTS:

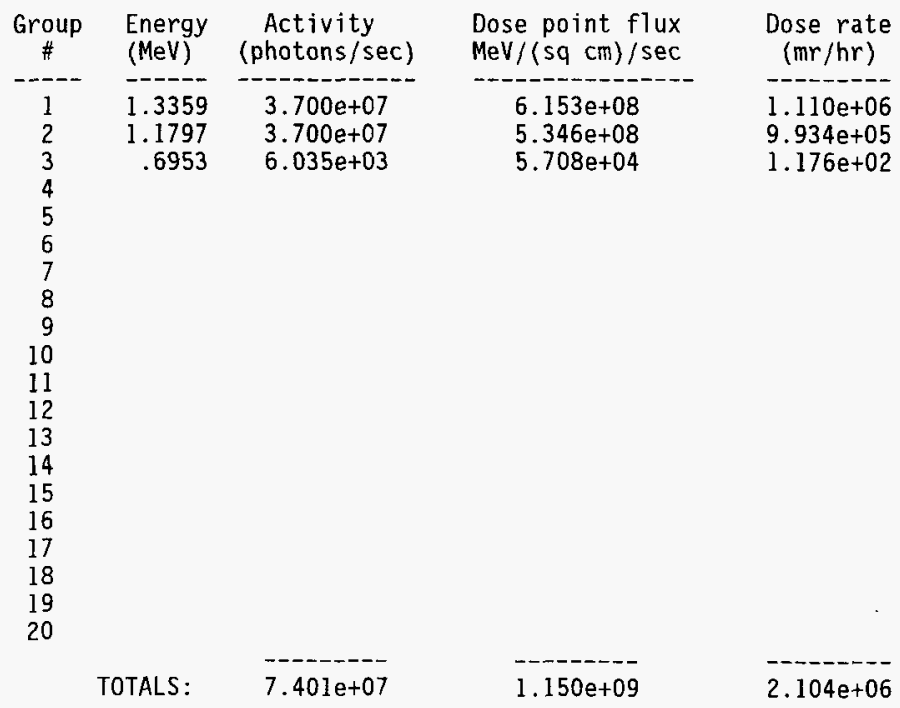




\section{WHC-SD-WM-CN-050 Rev 0}

Microshield 3.12

$== \pm= \pm===== \pm==$ (Westinghouse Hanford Company - \#197)
Page : 1
File : COlOCM.MSH
Run date: July 11, 1996
Run time: $3: 20 \mathrm{p} . \mathrm{m}$.
File Ref:
Date:
By:
Checked:

CASE: $10 \mathrm{~cm}$

GEOMETRY 14: Infinite slab source - slab shields

Distance to detector.................... X

Source slab thickness.................... Tl

Thickness of second shield............... T2

110.

100 .

10.

$\mathrm{cm}$.

"

MATERIAL DENSITIES $(\mathrm{g} / \mathrm{cc})$ :

\begin{tabular}{lll} 
Material & Source & Shie1d 2 \\
\hline Air & & \\
Aluminum & & \\
Carbon & & \\
Concrete & & \\
Hydrogen & & \\
Iron & & \\
Lead & & \\
Lithium & & \\
Nickel & & \\
Tin & & \\
Titanium & & \\
Tungsten & & \\
Urania & & \\
Uranium & & \\
Water & 1.50 & 1.50 \\
Zirconium & &
\end{tabular}


BUILDUP FACTOR: based on TAYLOR method.

Using the characteristics of the materials in shield 1.

\section{INTEGRATION PARAMETERS:}

None - analytically integrated.

SOURCE NUCLIDES:

\begin{tabular}{llllll} 
Nuclide & Curies & Nuclide & Curies & Nuclide & Curies \\
\hdashline $\mathrm{Ba}-137 \mathrm{~m}$ & $0.0000 \mathrm{e}+00$ & $\mathrm{C} 0-60$ & $1.0000 \mathrm{e}-03$ & $\mathrm{Cs}-137$ & $0.0000 \mathrm{e}+00$ \\
$\mathrm{~N}-16$ & $0.0000 \mathrm{e}+00$ & & & &
\end{tabular}

RESULTS:

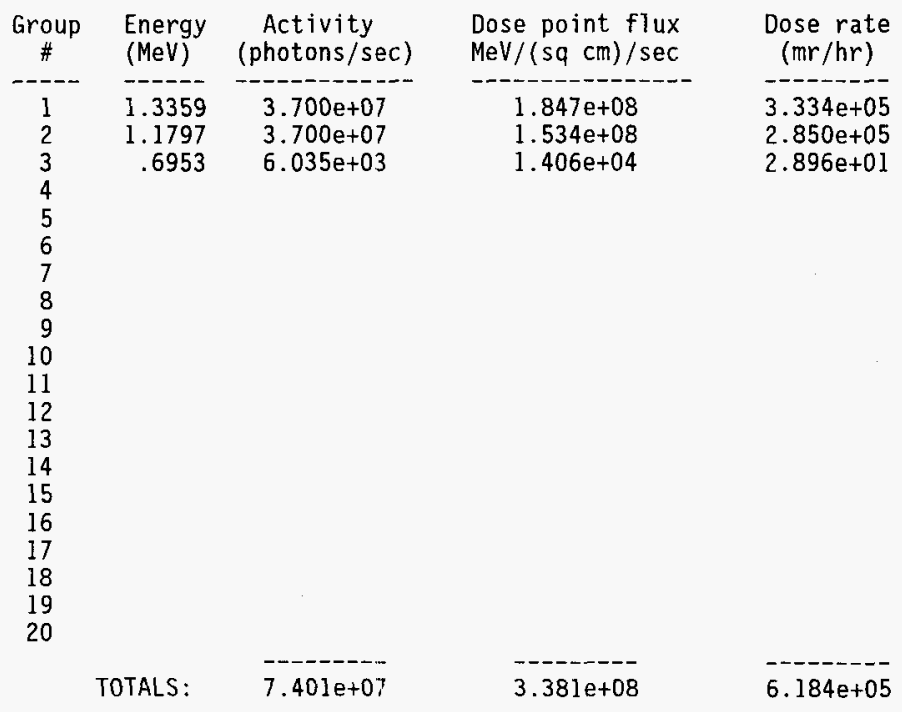




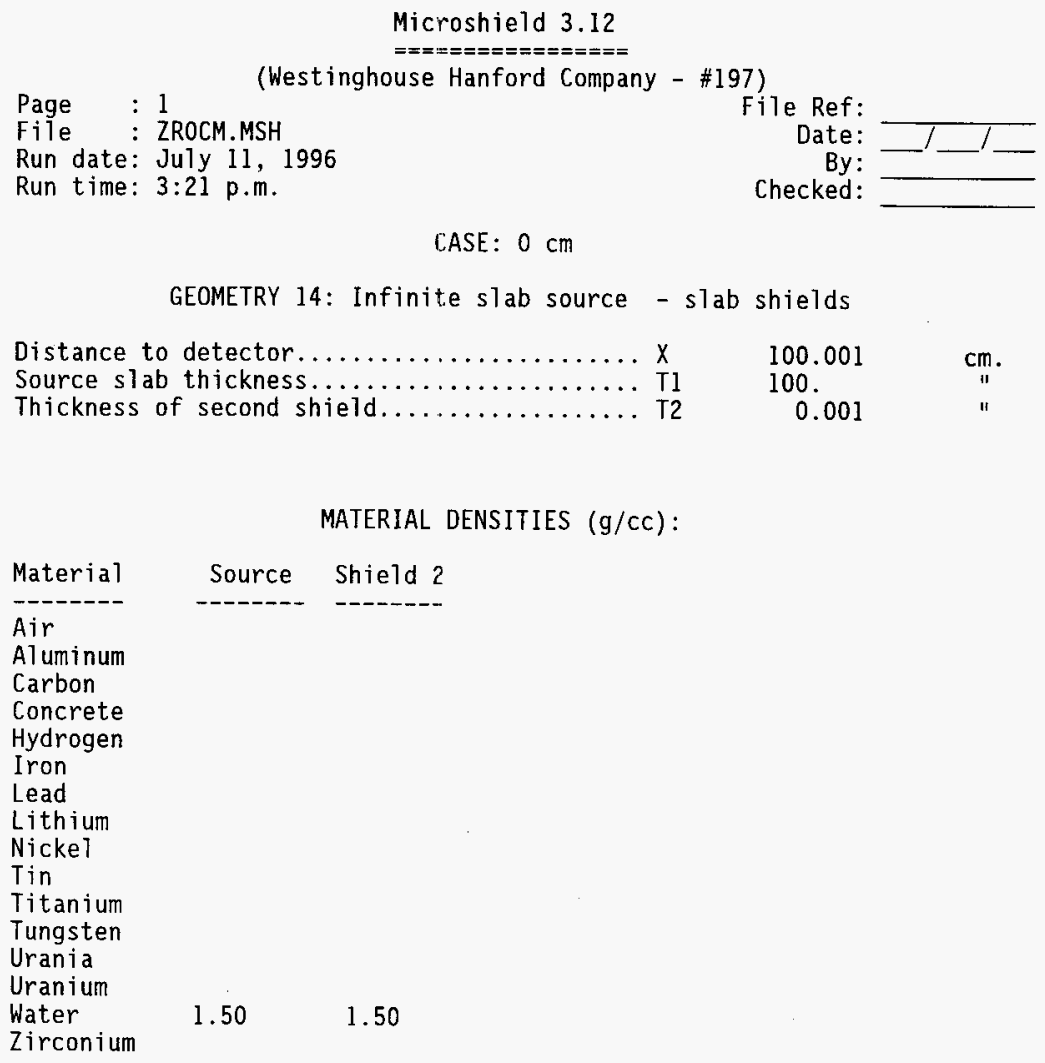




\section{WHC-SD-WM-CN-050 Rev 0}

Page 2

File: ZROCM.MSH

CASE : $0 \mathrm{~cm}$

BUILDUP FACTOR: based on TAYLOR method.

Using the characteristics of the materials in shield 1 .

INTEGRATION PARAMETERS:

None - analytically integrated.

SOURCE NUCLIDES:

Zr-95: $1.0000 \mathrm{e}-03$ curies

RESULTS:

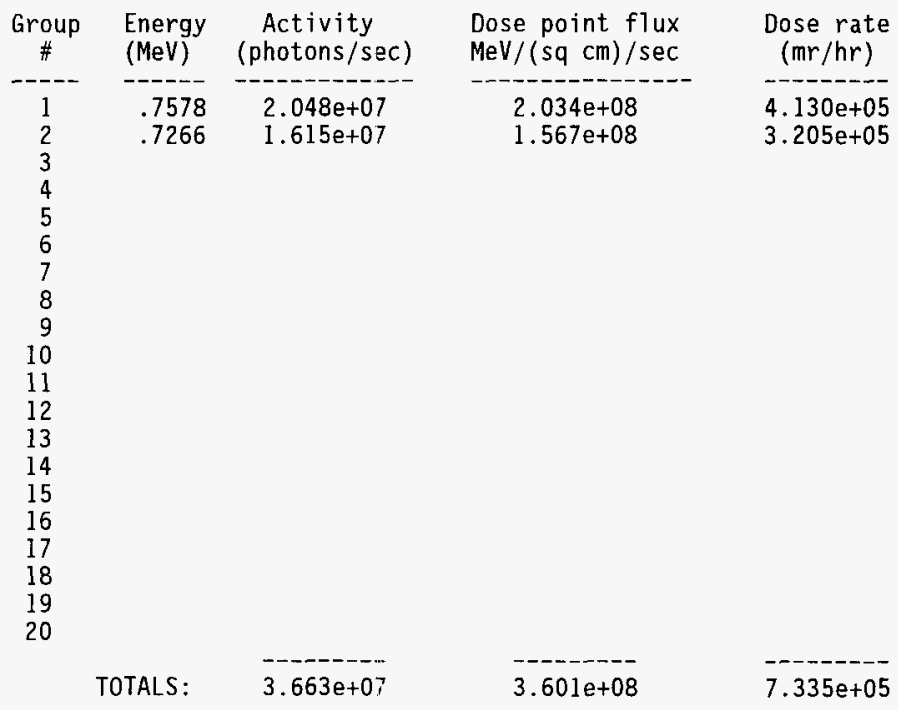


Microshield 3.12

$===x==x====x==x$

Page : 1

File : ZRIOCM.MSH

(Westinghouse Hanford Company - \#197)

Run date: July 11, 1996

Run time: 3:2l p.m.

File Ref:

Date:

By:

Checked:

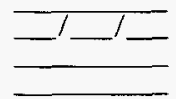

CASE : $10 \mathrm{~cm}$

GEOMETRY 14: Infinite slab source - slab shields

Distance to detector............... 110 .

Source $s l a b$ thickness..................... T1 100.

Thickness of second shield............. T2 10.

MATERIAL DENSITIES $(g / c c)$ :

\begin{tabular}{lll} 
Material & Source & Shield 2 \\
\hline Air & & \\
Aluminum & & \\
Carbon & & \\
Concrete & \\
Hydrogen & \\
Iron & \\
Lead & \\
Lithium & \\
Nickel & \\
Tin & \\
Titanium & \\
Tungsten & & \\
Urania & & \\
Uranium & & \\
Water & 1.50 & 1.50 \\
Zirconium & & \\
& &
\end{tabular}


WHC-SO-WM-CN-050 Rev 0

Page 2

File: ZR10CM.MSH

CASE : $10 \mathrm{~cm}$

BUILDUP FACTOR: based on TAYLOR method.

Using the characteristics of the materials in shield 1 .

INTEGRATION PARAMETERS:

None - analytically integrated.

SOURCE NUCLIDES:

Zr-95: 1.0000 e-03 curies

RESULTS:

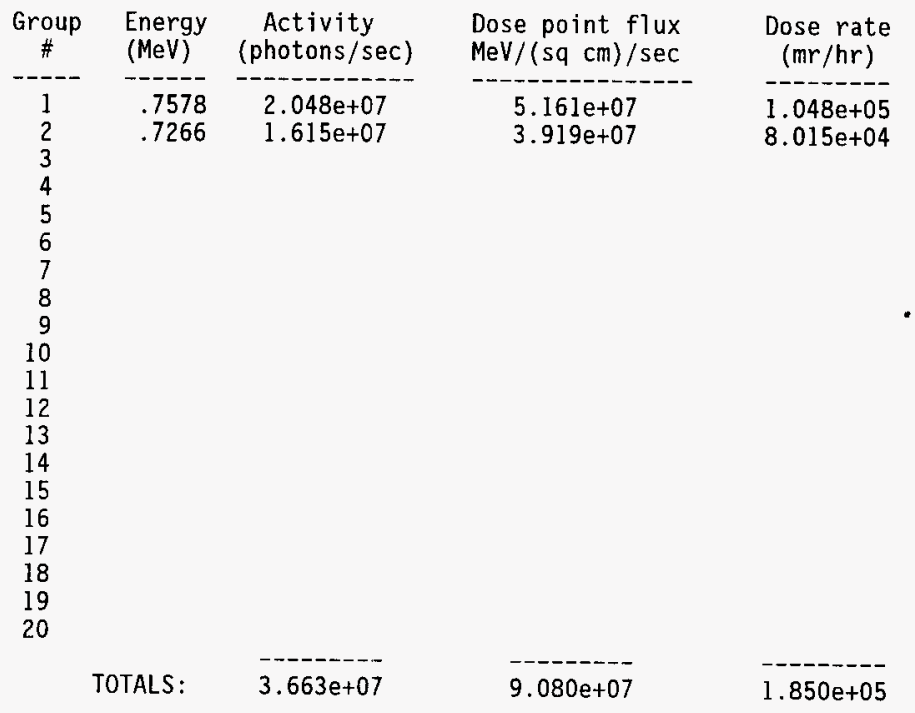


WHC-SD-WM-CN-050 Rev 0

Microshield 3.12

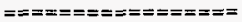

Page : 1

File : SBOCM.MSH

Run date: July 11,1996

Run time: $3: 22$ p.m.

(Westinghouse Hanford Company - \#197) File Ref

Date:

By:

Checked:

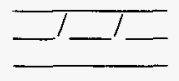

CASE : $0 \mathrm{~cm}$

GEOMETRY 14: Infinite slab source - slab shields

Distance to detector.................... X

Source slab thickness................... Tl

Thickness of second shield.

100.001

100 .

0.001

cm.

MATERIAL DENSITIES $(\mathrm{g} / \mathrm{cc})$ :

\begin{tabular}{lcc} 
Material & Source & Shield 2 \\
\hline Air & & \\
Aluminum & & \\
Carbon & & \\
Concrete & & \\
Hydrogen & & \\
Iron & & \\
Lead & & \\
Lithium & & \\
Nicke1 & & \\
Tin & & \\
Titanium & & \\
Tungsten & & \\
Urania & & \\
Uranium & & \\
Water & 1.50 & 1.50 \\
Zirconium & &
\end{tabular}


BUILDUP FACTOR: based on TAYLOR method.

Using the characteristics of the materials in shield 1 .

INTEGRATION PARAMETERS:

None - analytically integrated.

SOURCE NUCLIDES:

Sb-125: 1.0000e-03 curies

RESULTS:

\begin{tabular}{|c|c|c|c|c|}
\hline Group & $\begin{array}{l}\text { Energy } \\
(\mathrm{MeV})\end{array}$ & $\begin{array}{c}\text { Activity } \\
\text { (photons/sec) }\end{array}$ & $\begin{array}{l}\text { Dose point flux } \\
\mathrm{MeV} /(\mathrm{sq} \mathrm{cm}) / \mathrm{sec}\end{array}$ & $\begin{array}{c}\text { Dose rate } \\
(\mathrm{mr} / \mathrm{hr})\end{array}$ \\
\hline $\begin{array}{r}1 \\
2 \\
3 \\
4 \\
5 \\
6 \\
7 \\
8 \\
9 \\
10 \\
11 \\
12 \\
13 \\
14 \\
15 \\
16 \\
17 \\
18 \\
19 \\
20\end{array}$ & $\begin{array}{l}.6371 \\
.6016 \\
.4379 \\
.4141 \\
.3828 \\
.3203 \\
.2139 \\
.1797 \\
.1641 \\
.1172\end{array}$ & $\begin{array}{l}4.859 e+06 \\
8.434 e+06 \\
1.479 e+07 \\
6.728 e+04 \\
5.534 e+05 \\
1.543 e+05 \\
2.580 e+05 \\
2.617 e+06 \\
2.580 e+04 \\
9.658 e+04\end{array}$ & $\begin{array}{l}4.389 \mathrm{e}+07 \\
7.490 \mathrm{e}+07 \\
1.032 \mathrm{e}+08 \\
4.346 \mathrm{e}+05 \\
3.199 \mathrm{e}+06 \\
6.958 \mathrm{e}+05 \\
6.708 \mathrm{e}+05 \\
5.397 \mathrm{e}+06 \\
4.718 \mathrm{e}+04 \\
1.130 \mathrm{e}+05\end{array}$ & $\begin{array}{l}9.116 \mathrm{e}+04 \\
1.550 \mathrm{e}+05 \\
2.112 \mathrm{e}+05 \\
8.909 \mathrm{e}+02 \\
6.574 \mathrm{e}+03 \\
1.413 \mathrm{e}+03 \\
1.259 \mathrm{e}+03 \\
9.699 \mathrm{e}+03 \\
8.307 \mathrm{e}+01 \\
1.784 \mathrm{e}+02\end{array}$ \\
\hline & OTALS: & $3.186 \mathrm{e}+07$ & $2.326 \mathrm{e}+08$ & $4.774 \mathrm{e}+05$ \\
\hline
\end{tabular}


WHC-SD-WM-CN-050 Rev 0

Microshield 3.12

$===============$

File : SBIOCM.MSH

(Westinghouse Hanford Company - \#197)

Page : 1

Run date: July 11, 1996

Run time: $3: 23$ p.m.

File Ref:

Date:

By:

Checked:

CASE : $10 \mathrm{~cm}$

GEOMETRY 14: Infinite slab source - slab shields

Distance to detector.................... X

Source slab thickness..................... Tl

Thickness of second shield................ T2

110.

100.

10.

cm.

"

MATERIAL. DENSITIES $(\mathrm{g} / \mathrm{cc})$ :

\begin{tabular}{lll} 
Material & Source & Shield 2 \\
\hline Air & & \\
A7uminum & & \\
Carbon & & \\
Concrete & & \\
Hydrogen & & \\
Iron & & \\
Lead & & \\
Lithium & & \\
Nicke1 & & \\
Tin & & \\
Titanium & & \\
Tungsten & & \\
Urania & & \\
Uranium & & \\
Water & 1.50 & 1.50 \\
Zirconium & &
\end{tabular}


WHC-SD-WM-CN-050 Rev 0

Page 2

CASE: $10 \mathrm{~cm}$

File: SB1OCM.MSH

BUILDUP FACTOR: based on TAYLOR method.

Using the characteristics of the materials in shield 1 .

INTEGRATION PARAMETERS:

None - analytically integrated.

SOURCE NUCLIDES:

Sb-125: $1.0000 \mathrm{e}-03$ curies

RESULTS:

\begin{tabular}{|c|c|c|c|c|}
\hline$\underset{\#}{\text { Group }}$ & $\begin{array}{l}\text { Energy } \\
(\mathrm{MeV})\end{array}$ & $\begin{array}{c}\text { Activity } \\
\text { (photons/sec) }\end{array}$ & $\begin{array}{l}\text { Dose point flux } \\
\mathrm{MeV} /(\mathrm{sq} \mathrm{cm}) / \mathrm{sec}\end{array}$ & $\begin{array}{l}\text { Dose rate } \\
(\mathrm{mr} / \mathrm{hr})\end{array}$ \\
\hline $\begin{array}{r}1 \\
2 \\
3 \\
4 \\
5 \\
6 \\
7 \\
8 \\
9 \\
10 \\
11 \\
12 \\
13 \\
14 \\
15 \\
16 \\
17 \\
18 \\
19 \\
20\end{array}$ & $\begin{array}{l}.6371 \\
.6016 \\
.4379 \\
.4141 \\
.3828 \\
.3203 \\
.2139 \\
.1797 \\
.1641 \\
.1172\end{array}$ & $\begin{array}{l}4.859 e+06 \\
8.434 e+06 \\
1.479 e+07 \\
6.728 e+04 \\
5.534 e+05 \\
1.543 e+05 \\
2.580 e+05 \\
2.617 e+06 \\
2.580 e+04 \\
9.658 e+04\end{array}$ & $\begin{array}{l}1.053 \mathrm{e}+07 \\
1.774 \mathrm{e}+07 \\
2.126 \mathrm{e}+07 \\
8.672 \mathrm{e}+04 \\
6.076 \mathrm{e}+05 \\
1.181 \mathrm{e}+05 \\
8.777 \mathrm{e}+04 \\
6.307 \mathrm{e}+05 \\
5.197 \mathrm{e}+03 \\
9.780 \mathrm{e}+03\end{array}$ & $\begin{array}{l}2.187 e+04 \\
3.670 e+04 \\
4.349 e+04 \\
1.778 e+02 \\
1.248 e+03 \\
2.398 e+02 \\
1.647 e+02 \\
1.133 e+03 \\
9.150 e+00 \\
1.544 e+01\end{array}$ \\
\hline & TALS: & $3.186 \mathrm{e}+07$ & $5.107 \mathrm{e}+07$ & $1.050 \mathrm{e}+$ \\
\hline
\end{tabular}


Microshield 3.12

$===============$

(Westinghouse Hanford Company - \#197)

Page : l

Run date: July 11, 1996

Run time: $3: 24$ p.m.

File Ref:

Date:

By:

Checked:

CASE : $0 \mathrm{~cm}$

GEOMETRY 14: Infinite slab source - slab shields

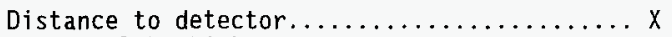

Source slab thickness.................... T1

Thickness of second shield............... T2

100.001

100.

0.001

$\mathrm{cm}$.

II

MATERIAL DENSITIES $(\mathrm{g} / \mathrm{cc})$ :

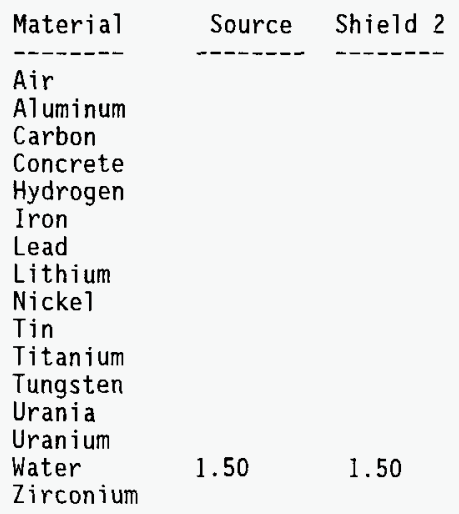


WHC-SD-WM-CN-050 Rev 0

Page 2

CASE: $0 \mathrm{~cm}$

File: CSOCM.MSH

BUILDUP FACTOR: based on TAYLOR method.

Using the characteristics of the materials in shield 1 .

INTEGRATION PARAMETERS:

None - analytically integrated.

SOURCE NUCLIDES:

Cs-134: $1.0000 \mathrm{e}-03$ curies

RESULTS:

\begin{tabular}{|c|c|c|c|c|}
\hline Group & $\begin{array}{l}\text { Energy } \\
(\mathrm{MeV})\end{array}$ & $\begin{array}{c}\text { Activity } \\
\text { (photons/sec) }\end{array}$ & $\begin{array}{l}\text { Dose point flux } \\
\mathrm{MeV} /(\mathrm{sq} \mathrm{cm}) / \mathrm{sec}\end{array}$ & $\begin{array}{c}\text { Dose rate } \\
(\mathrm{mr} / \mathrm{hr})\end{array}$ \\
\hline $\begin{array}{r}1 \\
2 \\
3 \\
4 \\
5 \\
6 \\
7 \\
8 \\
9 \\
10 \\
11 \\
12 \\
13 \\
14 \\
15 \\
16 \\
17 \\
18 \\
19 \\
20\end{array}$ & $\begin{array}{r}1.3672 \\
1.1641 \\
1.0391 \\
.8047 \\
.7891 \\
.6016 \\
.5703 \\
.4766 \\
.2734\end{array}$ & $\begin{array}{l}1.125 \mathrm{e}+06 \\
6.660 \mathrm{e}+05 \\
3.700 \mathrm{e}+05 \\
3.230 \mathrm{e}+06 \\
3.160 \mathrm{e}+07 \\
3.611 \mathrm{e}+07 \\
8.810 \mathrm{e}+06 \\
5.402 \mathrm{e}+05 \\
1.310 \mathrm{e}+04\end{array}$ & $\begin{array}{l}1.923 e+07 \\
9.493 e+06 \\
4.672 e+06 \\
3.307 e+07 \\
3.206 e+08 \\
3.207 e+08 \\
7.637 e+07 \\
4.239 e+06 \\
4.739 e+04\end{array}$ & $\begin{array}{l}3.451 e+04 \\
1.770 e+04 \\
8.945 e+03 \\
6.645 e+04 \\
6.464 e+05 \\
6.635 e+05 \\
1.575 e+05 \\
8.650 e+03 \\
9.399 e+01\end{array}$ \\
\hline & OTALS: & $8.246 e+07$ & $7.884 \mathrm{e}+08$ & $1.604 \mathrm{e}+06$ \\
\hline
\end{tabular}


Microshield 3.12

$================$

Page : 1

File : CS10CM.MSH

Run date: July 11, 1996

Run time: $3: 25$ p.m.
(Westinghouse Hanford Company - \#197)
FiTe Ref:

Date:

By:

Checked:

CASE : $10 \mathrm{~cm}$

GEOMETRY 14: Infinite slab source - slab shields

Distance to detector................ 110 .

Source slab thickness.................... Tl 100

Thickness of second shield............... T2 10.

Cif

II

MATERIAL DENSITIES $(g / c c)$ :

\begin{tabular}{lll} 
Material & Source & Shield 2 \\
\hline Air & & \\
Aluminum & & \\
Carbon & \\
Concrete & \\
Hydrogen & \\
Iron & \\
Lead & \\
Lithium & \\
Nickel & \\
Tin & \\
Titanium & \\
Tungsten & \\
Urania & & \\
Uranium & \\
Water & 1.50 & \\
Zirconium & &
\end{tabular}


WHC-SD-WM-CN-050 Rev 0

Page 2

File: CS10CM.MSH

CASE : $10 \mathrm{~cm}$

BUILDUP FACTOR: based on TAYLOR method.

Using the characteristics of the materials in shield 1 .

INTEGRATION PARAMETERS:

None - analytically integrated.

SOURCE NUCLIDES:

Cs-134: $1.0000 \mathrm{e}-03$ curies

RESULTS:

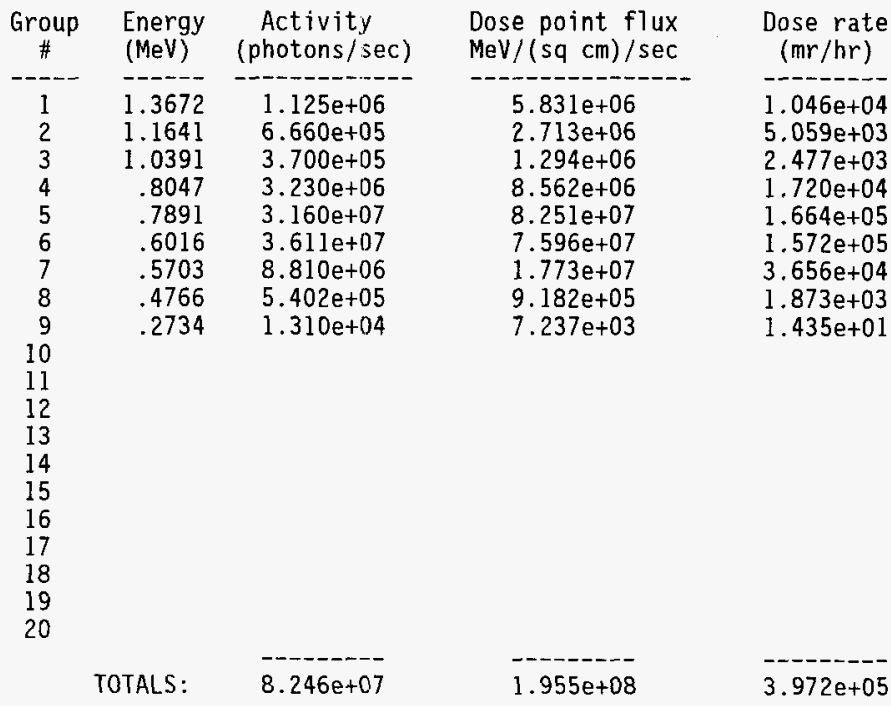


WHC-SD-WM-CN-050 Rev 0

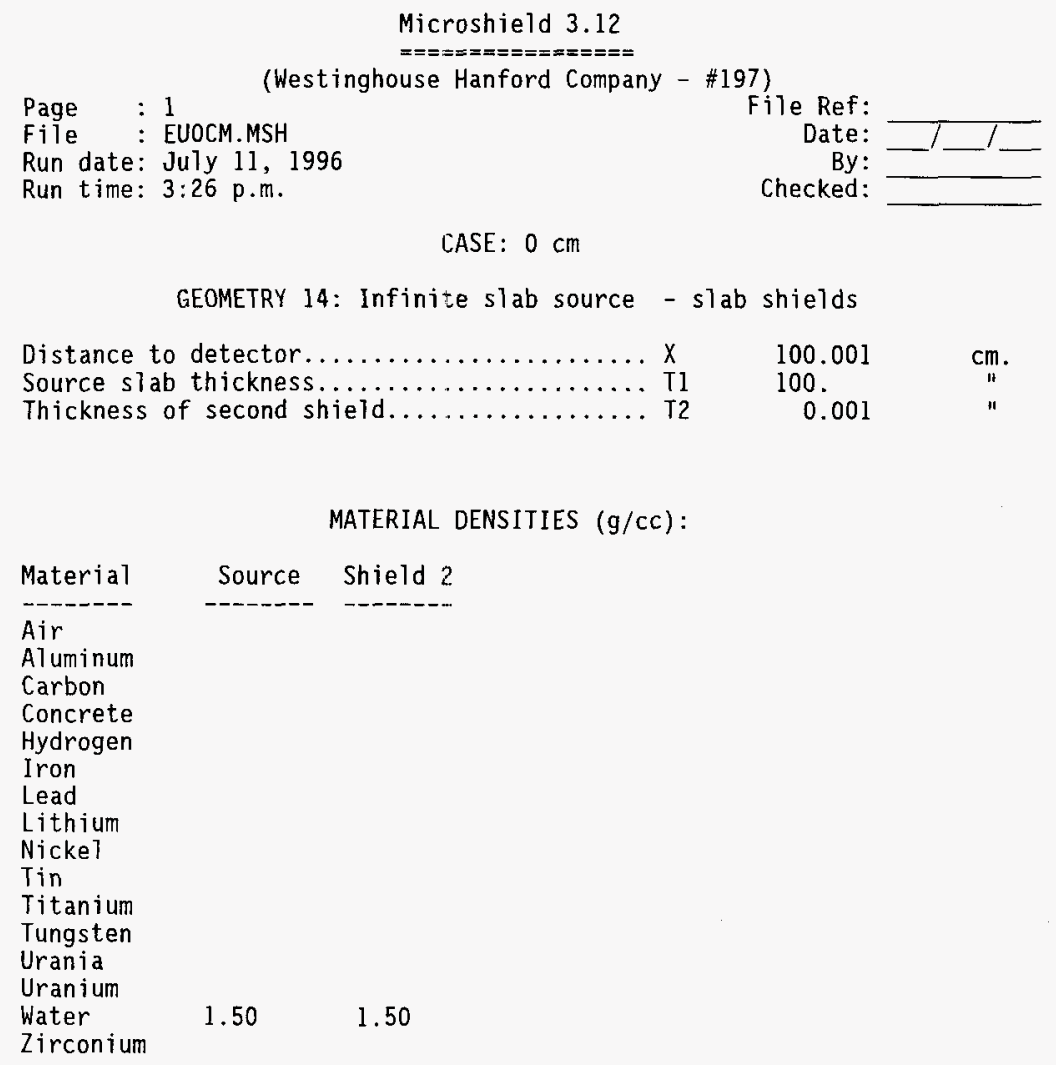


WHC-SD-WM-CN-050 Rev 0

Page 2

File: EUOCM.MSH

CASE : $0 \mathrm{~cm}$

BUILDUP FACTOR: based on TAYLOR method.

Using the characteristics of the materials in shield 1 .

INTEGRATION PARAMETERS:

None - analytically integrated.

SOURCE NUCLIDES :

Eu-154: 1.0000e-03 curies

RESULTS:

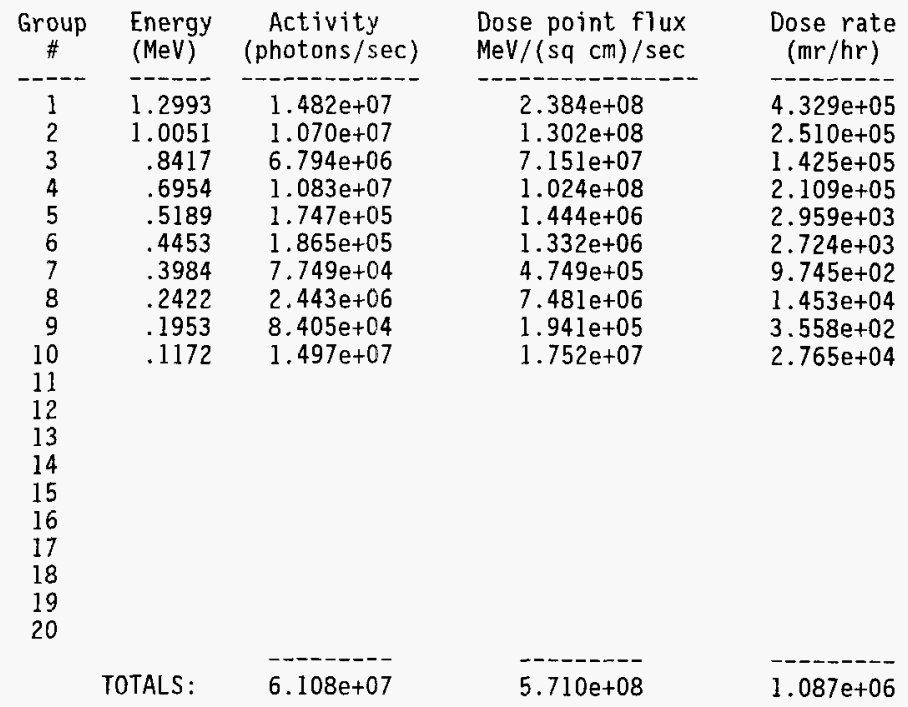


WHC-SD-WM-CN-050 Rev 0

Microshield 3.12

$===============-$

File : EUIOCM.MSH

Run date: July 11, 1996

Run time: $3: 26$ p.m.

Fjle Ref:

Date:

By:

Checked:

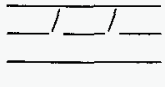

GEOMETRY 14: Infinite slab source - slab shields

Distance to detector................. X

Source slab thickness..................... T1

Thickness of second shield................ T2

110 .

100.

10 .

$\mathrm{cm}$.

"11

"

MATERIAL DENSITIES $(g / C C)$ :

Material Source Shield 2

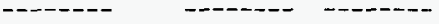

Air

Aluminum

Carbon

Concrete

Hydrogen

Iron

Lead

Lithium

Nicke]

Tin

Titanium

Tungsten

Urania

Uranium

Water

1.50

1.50

Zirconium 
Page 2

CASE: $10 \mathrm{~cm}$

BUILDUP FACTOR: based on TAYLOR method.

Using the characteristics of the materials in shield 1 .

INTEGRATION PARAMETERS:

None - analytically integrated.

SOURCE NUCLIDES:

Eu-154: 1.0000e-03 curies

RESULTS:

\begin{tabular}{|c|c|c|c|c|}
\hline Group & $\begin{array}{l}\text { Energy } \\
(\mathrm{MeV})\end{array}$ & $\begin{array}{c}\text { Activity } \\
\text { (photons/sec) }\end{array}$ & $\begin{array}{l}\text { Dose point flux } \\
\operatorname{MeV} /(\mathrm{sq} \mathrm{cm}) / \mathrm{sec}\end{array}$ & $\begin{array}{c}\text { Dose rate } \\
(\mathrm{mr} / \mathrm{hr})\end{array}$ \\
\hline $\begin{array}{r}1 \\
2 \\
3 \\
4 \\
5 \\
6 \\
7 \\
8 \\
9 \\
10 \\
11 \\
12 \\
13 \\
14 \\
15 \\
16 \\
17 \\
18 \\
19 \\
20\end{array}$ & $\begin{array}{r}-.0- \\
1.2993 \\
1.0051 \\
.8417 \\
.6954 \\
.5189 \\
.4453 \\
.3984 \\
.2422 \\
.1953 \\
.1172\end{array}$ & $\begin{array}{l}1.482 \mathrm{e}+07 \\
1.070 \mathrm{e}+07 \\
6.794 \mathrm{e}+06 \\
1.083 \mathrm{e}+07 \\
1.747 \mathrm{e}+05 \\
1.865 \mathrm{e}+05 \\
7.749 \mathrm{e}+04 \\
2.443 \mathrm{e}+06 \\
8.405 \mathrm{e}+04 \\
1.497 \mathrm{e}+07\end{array}$ & $\begin{array}{l}7.075 \mathrm{e}+07 \\
3.573 \mathrm{e}+07 \\
1.870 \mathrm{e}+07 \\
2.524 \mathrm{e}+07 \\
3.239 \mathrm{e}+05 \\
2.770 \mathrm{e}+05 \\
9.274 \mathrm{e}+04 \\
1.053 \mathrm{e}+06 \\
2.407 \mathrm{e}+04 \\
1.516 \mathrm{e}+06\end{array}$ & $\begin{array}{l}1.285 e+05 \\
6.889 e+04 \\
3.728 e+04 \\
5.197 e+04 \\
6.640 e+02 \\
5.664 e+02 \\
1.903 e+02 \\
2.046 e+03 \\
4.412 e+01 \\
2.393 e+03\end{array}$ \\
\hline & OTALS: & $6.108 \mathrm{e}+07$ & $1.537 \mathrm{e}+08$ & $2.925 \mathrm{e}+0$ \\
\hline
\end{tabular}




\section{CHECKLIST FOR PEER REVIEW}

Document Reviewed: VERIFICATION OF DST ENERGY DEPOSITION RATES AND DEVELOPMENT OF CORRECTION FOR GAMMA LOSSES, D.A. Himes, $7 / 8 / 96$

Scope of Review: entire document

Yes No NA

[ ] [ ] [X] * Previous reviews complete and cover analysis, up to scope of this review, with no gaps.

[X] [ ] [ ] Problem completely defined.

[][]$[x]$ Accident scenarios developed in a clear and logical manner.

[X] [ ] [ ] Necessary assumptions explicitly stated and supported.

[x] [ ] [ ] Computer codes and data files documented.

[X] [ ] [ ] Data used in calculations explicitly stated in document.

[x] [ ] [ ] Data checked for consistency with original source information as applicable.

[X] [ ] [ ] Mathematical derivations checked including dimensional consistency of results.

[X] [ ] [ ] Models appropriate and used within range of validity or use outside range of established validity justified.

[x] [ ] [ ] Hand calculations checked for errors. Spreadsheet results should be spir thecktreated exactly the same as hand calculations.

[X] [ ] [ ] Software input correct and consistent with document reviewed.

[]$[$ [ ] Software output consistent with input and with results reported in document reviewed.

[ ] [ ] [X] Limits/criteria/guidelines applied to analysis results are appropriate and referenced. Limits/criteria/guidelines checked against references.

[ ] [ ] [X] Safety margins consistent with good engineering practices.

$[X]$ [ ] [ ] Conclusions consistent with analytical results and applicable limits.

[X] [ ] [ ] Results and conclusions address all points required in the problem statement.

[ ] [ ] [X] Format consistent with appropriate NRC Regulatory Guide or other

[ ] [X]* Review calculations, comments, and/or notes are attached.

[X] [ ] [ ] Document approved.

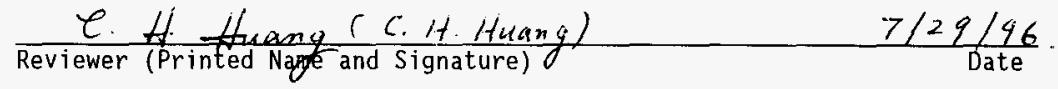

* Any calculations, comments, or notes generated as part of this review should be signed, dated and attached to this checklist. Such material should be labeled and recorded in such a manner as to be intelligible to a technically qualified third party. 


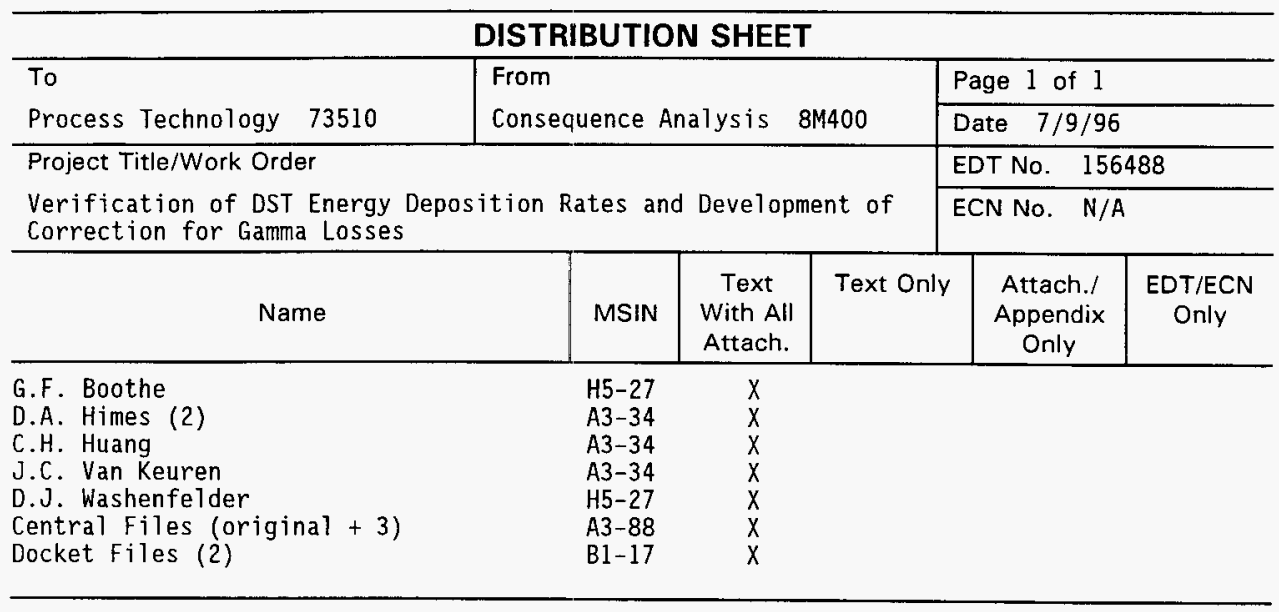

